\title{
DUALITIES IN M-THEORY AND BORN-INFELD THEORY
}

\author{
by

\section{Daniel Milton Brace} \\ B.A. (University of California, Berkeley) 1995 \\ M.A. (University of California, Berkeley) 1997 \\ A dissertation submitted in partial satisfaction of the \\ requirements for the degree of \\ Doctor of Philosophy in Physics \\ in the \\ GRADUATE DIVISION \\ of the \\ UNIVERSITY of CALIFORNIA, BERKELEY
}

Committee in charge:

Professor Bruno Zumino, Chair

Professor Hiroshi Ooguri

Professor Nicolai Y. Reshetikhin

Spring 2001 
The dissertation of Daniel Brace is approved:

\begin{tabular}{cc}
\hline Chair & Date \\
& \\
\hline & Date \\
\hline & Date
\end{tabular}

Date

University of California, Berkeley

Spring 2001 


\section{DUALITIES IN M-THEORY AND BORN-INFELD THEORY}

(c) 2001

by

Daniel Brace 


\begin{abstract}
DUALITIES IN M-THEORY AND BORN-INFELD THEORY

by
\end{abstract}

\author{
Daniel Brace \\ Doctor of Philosophy in Physics \\ University of California, Berkeley \\ Professor Bruno Zumino, Chair
}

We discuss two examples of duality. The first arises in the context of toroidal compactification of the discrete light cone quantization of M-theory. In the prescence of nontrivial moduli coming from the M-theory three form, it has been conjectured that the system is described by supersymmetric Yang-Mills gauge theory on a noncommutative torus. We are able to provide evidence for this conjecture, by showing that the dualities of this M-theory compactification, which correspond to T-duality in Type IIA string theory, are also dualities of the noncommutative supersymmetric Yang-Mills description. One can also consider this as evidence for the accuracy of the Matrix Theory description of M-theory in this background. The second type of duality is the self-duality of theories with $U(1)$ gauge fields. After discussing the general theory of duality invariance for theories with complex 
gauge fields, we are able to find a generalization of the well known $U(1)$ Born-Infeld theory that contains any number of gauge fields and which is invariant under the maximal duality group. We then find a supersymmetric extension of our results, and also show that our results can be extended to find Born-Infeld type actions in any even dimensional spacetime.

Chair Date


To my parents and my beautiful and intelligent sister 


\section{Acknowledgements}

To begin I would like to thank the members of my thesis committee: Hiroshi Ooguri, Nicolai Reshetikhin, and Bruno Zumino. In addition to serving on my thesis committee all three have gone out of their way to create a rich learning environment from which I have benefited greatly.

I would like to thank the fellow graduate students with whom I have spent many long hours working and hopefully just as many hours playing. Special thanks go to Micheal Grabe, Mathew McKerley, Bogdan Morariu, Brent Nelson, Harlan Robins, Jonathan Tannenhauser, and Celeste Winant.

Much of what I know about string theory was learned through discussions with my collaborator and friend Bogdan Morariu. I wish to thank him for all these discussions, in which much of the progress in our research was made.

I am forever indebted to my advisor, Bruno Zumino, for his guidance and the invaluable insights into physics which he has shared with me. I have also found much in the stories he has told me, some about physics or physicists and some not. It has been a privilege and a pleasure to work with him. 


\section{Contents}

1 Introduction and Outline $\quad 1$

1.1 Toroidal Compactification of the DLCQ of M-theory . . . . . . 6

1.2 Non-linear Electromagnetic Duality . . . . . . . . . . . . . . . 12

2 Super Yang-Mills on the Noncommutative Torus 15

2.1 Matrix Compactification ................ 16

2.2 Twisted Quantum Bundles on $T^{2} \ldots \ldots . \ldots . . . . . . . .23$

2.3 Twisted Quantum Bundles on Tori . . . . . . . . . . . . . 32

2.4 Adjoint Sections on Twisted Bundles . . . . . . . . . . . 35

2.5 Two and Three Dimensional Solutions . . . . . . . . . . . 41

2.6 Noncommutative Super Yang-Mills Action . . . . . . . . . . . . 47

3 Dualities of the Matrix Model from T-Duality of the Type II

$\begin{array}{ll}\text { String } & 51\end{array}$

$3.1 S O(3,3 \mid \mathbf{Z})$ Duality of Super Yang-Mills . . . . . . . . . . . 51

3.2 Target Space Duality . . . . . . . . . . . . 56

3.3 Projective Modules and Morita Equivalence . . . . . . . . . . 60

3.4 Gauge Transformations . . . . . . . . . . . . . . 66

4 Adding Ramond-Ramond Backgrounds $\quad 69$

4.1 Duality of Seven Dimensional Supergravity . . . . . . . . . . . 70 
4.2 T-duality of the Chern-Simon Type Terms . . . . . . . . . . 74

5 T-duality of the BPS Spectrum $\quad 82$

5.1 The $U(1)$ BPS Spectrum . . . . . . . . . . . . 84

5.2 Dual $U(n)$ BPS Spectrum . . . . . . . . . . . 86

6 Duality Invariance $\quad 92$

7 The Abelian Born-Infeld Action 103

7.1 Born-Infeld with Auxiliary Fields . . . . . . . . . . . . 103

7.2 Elimination of the Auxiliary Fields . . . . . . . . . . 106

7.3 Unilateral Matrix Equations . . . . . . . . . . . . 110

8 Extensions of the Born-Infeld Action $\quad 117$

8.1 Real Field Strengths . . . . . . . . . . . . . 117

8.2 Supersymmetric Theory . . . . . . . . . . . . 118

8.3 Extension to Arbitrary Even Dimensions . . . . . . . . . . . . 120

A Chiral Spinor Representations of $S O(d, d \mid \mathbf{Z}) \quad \mathbf{1 2 4}$

B Duality Invariants at Small Compactification Volume 130

C Parametrizations of Coset Spaces $G / H \quad 134$ 


\section{Chapter 1}

\section{Introduction and Outline}

String theory is the most promising candidate for a unified theory of gravity and the other interactions already present in the standard model of particle physics. Five consistent string theories are known to exist in ten dimensions. One of them is an open unoriented superstring theory called Type I. There are two closed superstring theories called Type IIA and Type IIB, depending on whether the GSO projection on left and right movers leads to a nonchiral or chiral spacetime theory. Finally there are two heterotic string theories, one with gauge group $S O(32)$ and one with gauge group $E_{8} \times E_{8}$. Lower dimensional string theories can then be obtained by compactification on a small manifold. Due to the large number of resulting theories it seemed that one of the initial promising features of string theory, its uniqueness, was lost. Furthermore, some of these theories do not have a unique classical vacuum, and perturbative corrections do not remove this degeneracy.

Some hope existed that nonperturbative corrections would select a specific vacuum, perhaps the one describing our world. However, only a perturbative formulation of these string theories is available, and it seemed until recently that no real progress could be made without a full nonperturbative formulation of 
string theory. Nevertheless, access to nonperturbative aspects of string theories was possible though an analysis of their low energy formulation as supergravity theories. Any relations between the five known string theories would have to be mirrored by their low energy descriptions. These relations are known as duality.

It has been known for some time that supergravity theories in four dimensions with extended supersymmetry are self-dual. For example, Cremmer and Julia [1] showed that the $N=8$ supergravity theory is invariant under a noncompact $E_{7}$ duality group. In fact, the requirements of self-duality and supersymmetry greatly facilitated finding the correct form of the $N=8$ supergravity Lagrangian.

A simple example of a self-dual theory is electromagnetism without matter. The equations of motion and the Bianchi identity for electromagnetism are

$$
\partial_{\mu} F^{\mu \nu}=0, \quad \partial_{\mu} \widetilde{F}^{\mu \nu}=0
$$

where $\widetilde{F}^{\mu \nu}=\frac{1}{2} \epsilon^{\mu \nu \alpha \beta} F_{\alpha \beta}$ is the Hodge dual of the field strength. One can see immediately, that the equations of motion and Bianchi identities remain valid when one replaces the field strength with its dual, and thus the theory is said to be self-dual. In fact, the theory is invariant under a continuous $S O(2)$ duality group which rotates the field strength and its dual.

Shortly after the appearance of duality in extended supergravity $[2,1]$ the theory of duality invariance of theories with abelian gauge fields was developed in $[3,4]$. However, there are very few examples of duality invariant interacting gauge theories where the Lagrangian is known in closed form. The most famous 
is the Born-Infeld theory $[5,6,7,8,9,10]$ and in the latter part of this thesis we study its generalization to more than one abelian gauge field.

The self-duality found in four dimensional extended supergravity can be understood as arising from duality structure of string theory, although in practice it is the duality structure of supergravity theories that has helped in understanding the dualities of string theory. In higher dimensions, a large number of nonperturbative supergravity solutions were found and used to access the strong coupling regime of string theory. This is possible in theories with extended supersymmetry by using the so called BPS states, which are states in small representations of the supersymmetry algebra. Since the dimension of these representations is an integer which cannot vary continuously, the states in these short multiplets cannot leave the representation as one changes continuous parameters, such as the coupling constant. Most importantly, for BPS states the supersymmetry algebra requires that certain relations between the masses and charges be satisfied, and this allows one to know the masses of these states even at strong coupling.

An important discovery due to Polchinski [11] was that the nonperturbative solutions of the low energy supergravity theories which carry Ramond-Ramond charges could be understood, in terms of the world sheet conformal field theory describing the string dynamics, as hypersurfaces in space-time where strings can end. These hypersurfaces are named Dirichlet branes or D-branes for short.

The surprise came in 1995 when it was realized $[12,13]$ that most of the known string theories at strong coupling were not new, strange and hard to understand 
theories, but in fact were described by other already known string theories. This relation between different string theories is known as string duality. Evidence for these dualities can be obtained by trying to identify in one theory nonperturbative massive BPS states which, when followed to strong coupling, become light, and can be identified as the fundamental degrees of freedom of the dual theory. All the known string theories were unified into a web of theories with each theory at strong coupling or small volume equivalent to another theory at weak coupling or large volume. The relation between large and small volume theories had been known since the late nineteen-eighthes as target space duality or T-duality. For example Type IIA and Type IIB are T-dual to each other after toroidal compactification. A more complicated version of target space duality occurs for compactification on Calabi-Yau spaces and is known as mirror symmetry. A further unification discovered by Strominger [14] involves theories compactified on Calabi-Yau manifolds of different topology. String theory can move continuously from one such theory to another using the the conifold transition. This is a mechanism involving massless black hole condensation.

However, not all the corners of this web were described by known string theories. In one particular corner describing the strong coupling dual of Type IIA string theory there is an eleven dimensional theory whose low energy description was given by eleven dimensional supergravity. This is the theory with the largest allowed number of supercharges, but it is nonrenormalizable. It was conjectured that there exists a consistent eleven dimensional theory, called M-theory, such that 
its low energy is eleven dimensional supergravity. Sometimes the name M-theory is used to describe the whole web and sometimes only the strong coupling dual of the Type IIA string.

The main thrust of the first part of this thesis is the study of a certain compactification of M-theory, which we will describe shortly, in the presence of arbitrary moduli. The main mathematical ingredient in this study to which we turn next is noncommutative geometry. A classic mathematical result of Gel'fand states that compact topological spaces are in one-to-one correspondence with commutative $C^{*}$-algebras. In one direction, to a topological space $X$, one can associate the algebra of continuous functions $C(X)$. Conversely and rather nontrivially, the spectrum of a commutative $C^{*}$-algebra is equivalent to a compact topological space. This important result allows for a dual description of topological spaces and brings powerful algebraic methods into the realm of topology. On the other hand, if one drops the commutativity requirement, a $C^{*}$-algebra $\mathcal{A}$ describes what is called by correspondence a quantum space.

To illustrate, consider the algebra of functions on a two-torus $C\left(T^{2}\right)$. An arbitrary element $f$ of this algebra has the Fourier expansion

$$
f=\sum_{k, l \in \mathbf{Z}} f_{k, l} U_{1}^{k} U_{2}^{l}
$$

where $U_{i}=e^{\sigma_{i}}$ are the generators of this algebra. In general some restrictions are imposed on the $c$-number coefficients $f_{k, l}$ (such as they form a square-summable or rapidly decreasing sequence). Very importantly (1.1) is a global statement. In the 
opposite direction, if we know that all the algebra elements have the form (1.1), we immediately recognize that they can be identified with functions on a two-torus. Thus one can read the topological compact space from the commutative algebra.

Since $\sigma_{i}$ are local coordinates on the torus, the generators $U_{i}$ commute. Instead we can consider the algebra whose elements have the form (1.1) but with $U_{i}$ 's satisfying

$$
U_{1} U_{2}=e^{2 \pi i \theta} U_{2} U_{1}
$$

where $\theta$, also called the deformation parameter, is a real number. This algebra is known as the algebra of 'functions' on the noncommutative torus.

Just as one can define a field theory on a commutative space, one can also do so on a noncommutative space. The first part of this thesis is devoted to investigating the conjecture of Connes, Douglas and Schwarz, which states that supersymmetric Yang-Mills gauge theory on noncommutative tori provide a description of the toroidal compactification of the discrete light cone quantization of M-theory.

\subsection{Toroidal Compactification of the DLCQ of M-theory}

One of the strongests candidate descriptions of M-theory was provided by Banks, Fishler, Shenker and Susskind (BFSS) in [15]. Instead of thinking of the Matrix model as an auxiliary theory used for regulating a membrane theory, they conjectured that the large $n$ Matrix model is M-theory. Membranes are then obtained as low energy excitations, but the Matrix model also describes a host 
of other states such as M5-branes, or after compactification to lower dimensions, strings and D-branes. A further refinement of the BFSS conjecture was given by Susskind [16], who proposed that each momentum sector of the discrete light cone quantization (DLCQ) of M-theory is described by a maximally supersymmetric Matrix model with the momentum identified with the rank of the gauge group. The conjecture was further clarified by Sen and Seiberg [17, 18]. They used an infinite boost and a compensating rescaling to show that the DLCQ Hamiltonian of the original M-theory, where the light-cone variable is identified with period $L$, is given by the Hamiltonian of an auxiliary M-theory compactified on a vanishingly small space-like circle of radius $R$. This is then equivalent to a weakly coupled Type IIA string theory, which will be referred to, following Sen [19], as the auxiliary Type II string theory. At the same time, the original light-cone momentum is mapped into Ramond-Ramond D0 brane charge. The string coupling and string mass scale are given by the $R \rightarrow 0$ limit of

$$
g_{S}=M_{P}^{3 / 2}(L R)^{3 / 4}, m_{S}=M_{P}^{3 / 2} L^{3 / 4} R^{-1 / 4},
$$

where $M_{P}$ is the eleven dimensional Planck mass.

In this limit as proposed by Witten [20], and discussed extensively in [21], the excited string states decouple and the dynamics of $n$ D0 branes is determined by the maximally supersymmetric Matrix model [22, 23, 24].

Toroidal compactification of M-theory can be obtained by considering Matrix theory on the covering space of the torus and imposing a periodicity constraint on 
the dynamical variables $[15,25,26]$. The constrained system is formally equivalent to a $U(n)$ super Yang-Mills (SYM) gauge theory on a dual torus. On the other hand, upon compactification on a $d$-dimensional torus $T^{d}$, M-theory has additional moduli from the three form of eleven dimensional supergravity. Connes, Douglas and Schwarz [27], conjectured that these moduli correspond to the deformation parameters $\Theta_{i j}$ of a noncommutative super Yang-Mills (NCSYM) gauge theory. Further studies of this subject followed in $[28,29,30,31,32,33,34,35,36,37$, $38,39,40,41]$

In [27], where compactification on a two-torus was considered in some detail, it was suggested that the $S L(2, \mathbf{Z})$ noncommutative duality group of the NCSYM gauge theory $[42,43,44,45,46]$ corresponds to the T-duality in the DLCQ direction and one of the space-like compact directions of M-theory. However, Rieffel and Schwarz [34] later showed that NCSYM gauge theories on higher dimensional tori have an $S O(d, d \mid \mathbf{Z})$ duality, and conjectured that this is the realization, in the NCSYM theory, of the auxiliary Type II string theory T-duality.

In general, as will be extensively discussed in the first part of this thesis, two NCSYM theories are dual to each other if there exists an element $\Lambda$ of the duality group $S O(d, d \mid \mathbf{Z})$ with the block decomposition ${ }^{1}$

$$
\Lambda=\left(\begin{array}{ll}
\mathcal{A} & \mathcal{B} \\
\mathcal{C} & \mathcal{D}
\end{array}\right),
$$

\footnotetext{
${ }^{1}$ The $S O(d, d \mid \mathbf{Z})$ subgroup of the T-duality group $O(d, d \mid \mathbf{Z})$ is the subgroup that does not exchange Type IIA and IIB string theories.
} 
such that their defining parameters are related as follows

$$
\begin{aligned}
\bar{\Theta} & =(\mathcal{A} \Theta+\mathcal{B})(\mathcal{C} \Theta+\mathcal{D})^{-1}, \\
\bar{G}^{i j} & =(\mathcal{C} \Theta+\mathcal{D})_{k}^{i}(\mathcal{C} \Theta+\mathcal{D})_{l}^{j} G^{k l}, \\
\bar{g}_{S Y M}^{2} & =\sqrt{|\operatorname{det}(\mathcal{C} \Theta+\mathcal{D})|} g_{S Y M}^{2}, \\
\bar{\eta} & =S(\Lambda) \eta \\
\bar{\chi} & =S(\Lambda) \chi .
\end{aligned}
$$

Here $S(\Lambda)$ denotes the Weyl spinor representation of $\Lambda$. The deformation parameter of the noncommutative torus $\Theta$ is a $d$-dimensional antisymmetric matrix, $G^{i j}$ is the metric defining the torus of the NCSYM, and $g_{S Y M}$ is the gauge coupling constant. The integral chiral spinor $\eta$ contains the Chern numbers of the bundle. For example, for compactification on a three torus, $\eta$ contains the rank of the group and the magnetic fluxes, and for a four torus it also includes the instanton number. The chiral spinor $\chi$ in (1.8) determines the parameters of the Chern-Simon type terms which can be added to the NCSYM action. In the auxiliary Type IIA string theory, $\chi$ is closely related to the R-R moduli.

The relation (1.4) was first written in [34]. Equation (1.7) appeared in [38] where $S(\Lambda)$ was identified as a canonical transformation and was independently found in [47] and identified as a chiral spinor transformation. Equation (1.5) was implicit in [38] and was first written in [47] where equation (1.6) was also derived. Finally, deriving (1.8) was the main thrust of [48].

The first part of this thesis is an investigation of this duality conjecture and 
some of its consequences. In Chapter 2, an extension of the method used in $[36,40]$ will be employed to construct twisted bundles over two and three-tori. Then we will explain in some detail how to solve the boundary conditions for sections in the fundamental and adjoint quantum bundle. Using the special form of the transition functions in the given gauge, we will find different equivalent forms of the general solution for fundamental sections.

In Chapter 3, we will show explicitly how to construct an action of the duality group $S O(d, d \mid \mathbf{Z})$ on NCSYM theories. Under these duality transformations, the rank of the gauge group and the magnetic flux numbers transform together in a Weyl spinor representation, and the deformation parameters transform by fractional transformations. We will also obtain the transformation properties, under the duality group, of the gauge coupling and the metric. One can then directly compare these relations with the string theory T-duality predictions. We will then discuss the more abstract language of projective modules, as presented in [27] and references therein, and give the explicit map between this formulation and the more elementary formulation in $[36,47]$. We will also explain the notion of Morita equivalence $[42,27,34,38,39]$ applied to our specific case ${ }^{2}$. Finally, we will end the chapter with a discussion of the general theory of gauge transformations on the noncommutative torus and find an explicit gauge transformation that trivializes one of the transition functions. With trivial transition functions, T-duality

\footnotetext{
${ }^{2}$ For an expanded coverage of noncommutative geometry, see [66] and for a brief description see [67].
} 
transformations take the standard form, allowing us to interpret the expectation value of the gauge field as the location of the D-strings on the dual torus.

In Chapter 4, we will include nonvanishing Chern-Simon couplings. The values of the Chern-Simon couplings are determined by the Ramond-Ramond moduli of the compactification. One finds perfect agreement between the transformation properties of the couplings derived from NCSYM or the auxiliary Type II string theory.

In Chapter 5, we will study the BPS spectrum corresponding to electric fluxes of the noncommutative supersymmetric Yang-Mills (NCSYM) gauge theory [49] compactified on a torus. This gives a description of the BPS spectrum of the DLCQ of M-theory compactified on a dual torus. Since the spectrum is invariant under the T-duality group $O(d, d \mid \mathbf{Z})$, where $d$ is the dimension of the compactification torus, one can first calculate the spectrum in the simplest case, which corresponds to a NCSYM gauge theory on a trivial bundle. Then one can use a duality transformation to rewrite the result in terms of the defining parameters of a dual theory on a nontrivial bundle. Alternatively, one can also obtain this result directly by quantizing the free system of collective coordinates of the twisted $U(n)$ theory. To obtain the spectrum one has to mod out by gauge equivalent configurations and show that the zero modes of the gauge field live on a compact space, a torus. In the classical case, one can find a global gauge transformation whose sole effect is a shift in the zero mode of the gauge field. Then the electric fluxes which are the conjugate variables are integrally quantized. However, for 
a nonvanishing deformation parameter the gauge transformation also results in a finite space translation $[27,50,51,52]$. Then, just as for the electric charge of dyons [53], by a Witten-Olive type effect, the electric flux spectrum for states carrying momentum contains an additional term proportional to the deformation parameter. The spectrum obtained is in agreement with the spectrum conjectured in the literature and obtained by imposing U-duality invariance.

\subsection{Non-linear Electromagnetic Duality}

The remainder of this thesis is devoted to the study of self-duality. In Chapter 6 we present in some detail the theory of duality invariance for a theory of complex gauge fields with holomorphic duality transformations. This is an extension of the theory of duality invariance which was developed in $[3,4]$ and briefly discussed in [54]. However, the duality group can be larger than that presented in [54]. In fact, for a gauge theory with $n$ complex gauge fields, the largest possible duality group is $U(n, n)$. We also discuss how to obtain such a theory from a theory with a $U(n) \times U(n)$ duality group, which is the maximal compact subgroup of $U(n, n)$, by introducing an additional $n$-dimensional matrix valued scalar field.

In Chapter 7, we describe a Born-Infeld Lagrangian written in terms of auxiliary fields, with a $U(n, n)$ duality group. Its form is closely related to the Lagrangian introduced in $[55,56]$ but differs in two ways. We use a different reality structure for our fields and introduce a dynamical scalar field such that the duality group is extended to a noncompact group. We find that it is possible to eliminate 
the auxilliary fields using some new mathematical results concerning unilateral matrix equations. In particular, we show that certain solutions of unilateral matrix equations can be written as a sum of terms which are symmetric in the matrix coefficients as well as terms which are commutators.

In the theory with auxiliary fields, it does not seem possible to work with real gauge fields, but this can be done in the Lagrangian with the auxiliary fields eliminated. As will be shown in Chapter 8, this leads to a Born-Infeld theory with an $S p(2 n, \mathbb{R})$ duality group. This is the first example of an interacting gauge theory whose Lagrangian is known to all orders and whose duality group is as large as the duality group of the Maxwell theory with the same number of gauge fields. We go on to show how to supersymmetrize the Born-Infeld Lagrangian in the formulation with auxiliary fields. We also present the form without auxiliary fields of the supersymmetric Born-Infeld Lagrangian with a single gauge field and a scalar field. This theory is invariant under $S L(2, \mathbb{R})$ duality, which reduces to $U(1)$ duality if the value of the scalar field is suitably fixed. Versions of this theory without the scalar field were presented in $[57,58,59]$. Finally, we generalize our construction to arbitrary even dimensions by using antisymmetric tensor fields such that the rank of their field strength equals half the dimension of space-time. First, we consider theories with a $U(n, n)$ duality group using complex antisymmetric tensor fields; then we discuss theories with real antisymmetric tensor fields. These have an $S p(2 n, \mathbb{R})$ duality group if half of the space-time dimension is even and $O(n, n)$ if it is odd. The fact that the duality group depends on half the dimension 
of space-time was discussed earlier in [60, 61, 62, 63, 64]. 


\section{Chapter 2}

\section{Super Yang-Mills on the Noncommutative Torus}

In the first section, we review the standard toroidal Matrix compactification leading to a SYM gauge theory on the dual torus. Then we present the conjecture [27] that in the presence of nonvanishing NS antisymmetric moduli $B_{i j}$, the translation generators implementing the quotient conditions do not commute, such that Matrix compactification leads to a noncommutative super Yang-Mills gauge theory on a dual noncommutative torus.

In Section 2.3, we study adjoint quantum bundles on noncommutative tori of arbitrary dimension which admit a constant curvature connection which is not valued in the $s u(n)$ subalgebra and have transition functions of a special simple form.

In Section 2.4, we show how to expand the sections of the adjoint bundle of a $U(n)$ gauge theory in terms of matrix valued functions on a dual noncommutative torus. The dual deformation parameter $\Theta^{\prime}$ lies on the same $S O(d, d \mid \mathbf{Z})$ orbit as the original $\Theta$. We perform most of the calculations on tori of arbitrary dimension, but later we will concentrate on the two and three-tori.

In Section 2.5, we describe the quantum bundles corresponding to the two dimensional compactification and rewrite some of the known two dimensional re- 
lations in a form that admits immediate generalization to higher dimensions. We also give the solution for arbitrary adjoint bundles over three dimensional tori.

In Section 2.6, we consider the noncommutative SYM action on a twisted quantum bundle after a brief description of the quantum integral.

Finally, in Appendix A we prove a theorem showing that the chiral spinor representations of $S O(d, d \mid \mathbf{Z})$ are integral, and also show that the spinor representation of $S O(3,3 \mid \mathbf{Z})$ is in fact $S L(4, \mathbf{Z})$.

\subsection{Matrix Compactification}

In this section we present a review of Matrix theory compactification. In the limit of large string mass the dynamics of $n$ D0 branes, in uncompactified spacetime, is determined by the maximally supersymmetric Matrix Model action [22, 23, 24],

$$
\mathcal{S}^{D 0}=\frac{1}{2 g_{S}} \int d t \operatorname{tr}\left(\sum_{M} \dot{X}^{M} \dot{X}^{M}+\frac{1}{(2 \pi)^{2}} \sum_{M<N}\left[X^{M}, X^{N}\right]\left[X^{M}, X^{N}\right]+\text { fermions }\right) .
$$

This action is obtained by dimensional reduction of the ten dimensional $\mathcal{N}=1$ SYM gauge theory. Alternatively one could work with the IKKT functional [65] obtained by dimensionally reducing, in all directions including time, the Euclidean ten dimensional SYM action.

The compactification of Matrix theory on a $d$-dimensional torus is obtained by

considering an infinite number of D0 branes living on $\mathbf{R}^{d}$, the covering space of 
the torus, and then imposing the following quotient conditions [15, 25].

$$
\begin{aligned}
\mathcal{U}_{i}^{-1} X^{I} \mathcal{U}_{i} & =2 \pi e_{i}^{I}+X^{I}, i, I=1, \ldots, d, \\
\mathcal{U}_{i}^{-1} X^{a} \mathcal{U}_{i} & =X^{a}, a=d+1, \ldots, 9, \\
\mathcal{U}_{i}^{-1} \psi \mathcal{U}_{i} & =\psi .
\end{aligned}
$$

The last equation in (2.1) contains the quotient condition for fermions. Here $I$ runs over the compact directions, and the $e_{i}^{I}$ form a basis defining the compactification lattice. The $\mathcal{U}_{i}$ 's are unitary operators. One can define new matrix coordinates

$$
X^{i}=e_{I}^{i} X^{I}
$$

which obey the simpler quotient conditions

$$
\mathcal{U}_{i}^{-1} X^{j} \mathcal{U}_{i}=2 \pi \delta_{i}^{j}+X^{j}
$$

In terms of the new variables the action takes the form

$$
\begin{gathered}
\mathcal{S}^{D 0}=\frac{1}{2 g_{S}} \int d t \operatorname{Tr}\left(G_{i j} \dot{X}^{i} \dot{X}^{j}+\frac{1}{2} \frac{1}{(2 \pi)^{2}} G_{i j} G_{k l}\left[X^{i}, X^{k}\right]\left[X^{j}, X^{l}\right]+\right. \\
\sum_{a} \dot{X}^{a} \dot{X}^{a}+\frac{1}{(2 \pi)^{2}} \sum_{a} G_{i j}\left[X^{i}, X^{a}\right]\left[X^{j}, X^{a}\right]+ \\
\left.\frac{1}{(2 \pi)^{2}} \sum_{a<b}\left[X^{a}, X^{b}\right]\left[X^{a}, X^{b}\right]+\text { fermions }\right),
\end{gathered}
$$

where we have introduced the metric $G_{i j}=\sum_{I} e_{i}^{I} e_{j}^{I}$. In (2.3), the trace over infinite dimensional matrices is formally divided by the infinite order the quotient group $\mathbf{Z}^{d}$.

The original solution of the quotient condition assumed that the translation operators commute

$$
\left[\mathcal{U}_{i}, \mathcal{U}_{j}\right]=0
$$


The standard way to solve (2.2) is to introduce an auxiliary Hilbert space on which $X^{i}{ }^{i}$ s and $\mathcal{U}_{i}{ }^{\prime}$ s act. In the simplest case this is taken to be the space of functions on a $d$-dimensional torus taking values in $\mathbf{C}^{n}$. Then if one lets the $\mathcal{U}_{i}$ 's be the generators of the algebra of functions on the torus

$$
\mathcal{U}_{i}=e^{i \sigma_{i}}
$$

where $\sigma_{i}$ are coordinates on the covering space of the torus, the $X^{i}$ s satisfying (2.2) must be covariant derivatives

$$
X^{j}=-2 \pi i D^{j}=-2 \pi i\left(\partial^{j}-i A^{j}\left(\mathcal{U}_{k}\right)\right)
$$

The partial derivative is with respect to $\sigma_{j}$, and $A^{j}$ are $n$-dimensional hermitian matrices. The action (2.3) can be rewritten as a $d$-dimensional SYM action, by replacing the $X^{i}$ s with covariant derivatives as above, and rewriting the trace over the infinite dimensional matrices as

$$
\operatorname{Tr}=\int \frac{d^{d} \sigma}{(2 \pi)^{d}} \operatorname{tr}
$$

Here $\operatorname{tr}$ is an $n$-dimensional trace, and the new coordinates $\sigma_{i}$ are to be integrated from zero to $2 \pi$. The action becomes

$$
\begin{aligned}
\mathcal{S}^{D 0}= & \frac{(2 \pi)^{2-d}}{4 g_{S} \sqrt{\operatorname{det}\left(G^{i j}\right)}} \int d t \int d^{d} \sigma \sqrt{\operatorname{det}\left(G^{i j}\right)} \operatorname{tr}\left(G_{\mu \nu} G_{\xi \rho}\left[D^{\mu}, D^{\xi}\right]\left[D^{\nu}, D^{\rho}\right]-\right. \\
& \left.\sum_{a} G_{\mu \nu}\left[D^{\mu}, X^{a}\right]\left[D^{\nu}, X^{a}\right]+\sum_{a<b}\left[X^{a}, X^{b}\right]\left[X^{a}, X^{b}\right]+\text { fermions }\right),
\end{aligned}
$$

where the scalar fields $X^{a}$ have been rescaled by a factor of $2 \pi$. We have written 
the action in standard form ${ }^{1}$ so that one can read off the SYM gauge coupling

$$
g_{S Y M}^{2}=g_{S}(2 \pi)^{d-2} \sqrt{\operatorname{det}\left(G^{i j}\right)} .
$$

Thus the gauge coupling $g_{S Y M}^{2}$ equals the string coupling on the T-dual torus. The square root factor accounts for the expected dilaton shift under T-duality.

Following [27] we consider the general case when the unitary operators $\mathcal{U}_{i}$ do not commute. Consistency of the quotient conditions requires that the $\mathcal{U}_{i}$ 's must commute up to a phase

$$
\mathcal{U}_{i} \mathcal{U}_{j}=e^{-2 \pi i \Theta_{i j}} \mathcal{U}_{j} \mathcal{U}_{i}
$$

Connes, Douglas and Schwarz conjectured that the deformation parameters $\Theta$ correspond to certain moduli of the compactification of the DLCQ of M theory on tori. If $\gamma^{i j-}$ represents a three cycle wrapped around the transversal directions $x^{i}$ and $x^{j}$ and the light cone direction $x^{-}$, then

$$
\Theta_{i j}=\frac{1}{(2 \pi)^{3}} \int_{\gamma^{i j-}} C,
$$

where $C$ is the antisymmetric three form of eleven dimensional supergravity. Written in terms of the auxiliary type IIA string theory variables,

$$
\Theta_{i j}=\frac{1}{(2 \pi)^{2}} \int_{\gamma^{i j}} B,
$$

\footnotetext{
${ }^{1}$ Note that the positions of all the indices are switched. For example the metric has upper indices. This just reflects the performed T-duality under which the metric is replaced with the inverse metric. Another way to understand the index position is that T-duality is a canonical transformation which exchanges coordinates and momenta and therefore reverses the index structure.
} 
where $B$ is the NS two form.

Next we give a heuristic explanation of the noncommutativity of the translation generators (2.6). Consider for simplicity compactification on $T^{2}$ and assume that there is only one D0-brane. Then the covering space depicted in figure 2.1 contains an infinite number of $T^{2}$ cells labeled by two integers. The matrix element $X_{p q}^{i}$ is associated to the oriented string starting on the D0-brane at $q=\left(q_{1}, q_{2}\right)$ and ending on the D0-brane at $p=\left(p_{1}, p_{2}\right)$. We define the translation operators such that their matrix elements are given by

$$
\begin{aligned}
& \left(\mathcal{U}_{1}\right)_{p q}=\delta_{p_{1}, q_{1}-1} \delta_{p_{2}, q_{2}} \\
& \left(\mathcal{U}_{2}\right)_{p q}=\delta_{p_{1}, q_{1}} \delta_{p_{2}, q_{2}-1}
\end{aligned}
$$

The translation generators then commute since each acts trivially in one subspace. Next consider the effect of turning on the modulus $B=B_{12} d x^{1} d x^{2}$. Since the covering space is topologically trivial and $B_{12}$ is constant one can eliminate it by a gauge transformation

$$
B^{\prime}=B+d \Lambda
$$

where for example $\Lambda=B_{12} x_{2} d x^{1}$. Eliminating $B$ comes at a price. One must modify the operators $\mathcal{U}_{i}$ implementing the periodicity condition.

Just as the wave function of a charged particle changes under electromagnetic gauge transformations

$$
\psi^{\prime}(x)=e^{i \lambda(x)} \psi(x)
$$

the wave functional of strings $\Psi[\Gamma]$, where $\Gamma$ is the curve where the string is located, 


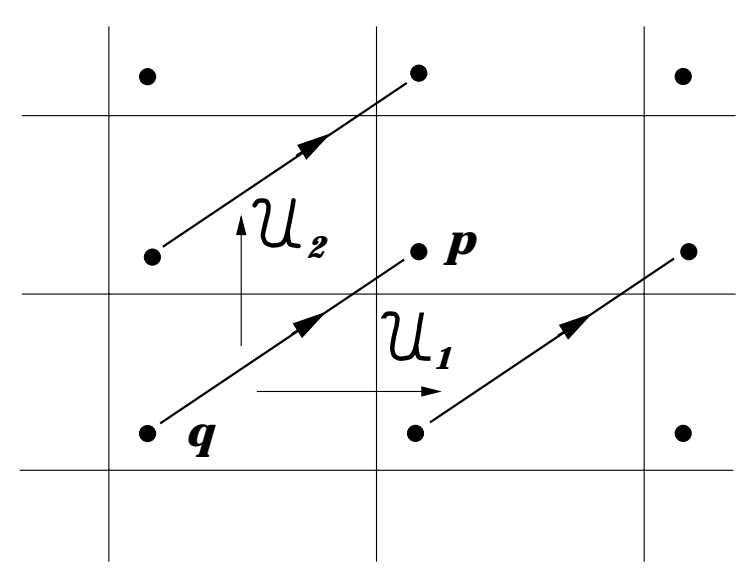

Figure 2.1: The covering space of $T^{2}$ with one D0-brane per cell

must transform under the gauge transformation (2.8) as

$$
\Psi^{\prime}[\Gamma]=e^{\frac{i}{2 \pi} \int_{\Gamma} \Lambda} \Psi[\Gamma]
$$

For the choice of $\Lambda$ made above the wave functional of each string acquires the phase $\exp \left(i / 2 \pi B_{12} \int x^{2} d x^{1}\right)$. The exponent is just the area under the string so the relative phase of strings related by a translation in the $x^{1}$ direction vanishes. For the $x^{2}$ direction the relative phase is $\exp \left(2 \pi i \Theta_{12}\left(p_{1}-q_{1}\right)\right)$. This requires a redefinition of the translation generators

$$
\begin{aligned}
\left(\mathcal{U}_{1}\right)_{p q} & =\delta_{p_{1} q_{1}-1} \delta_{p_{2} q_{2}} \\
\left(\mathcal{U}_{2}\right)_{p q} & =e^{-2 \pi i \Theta_{12} q_{1}} \delta_{p_{1} q_{1}} \delta_{p_{2} q_{2}-1}
\end{aligned}
$$

Then a direct computations shows that the $\mathcal{U}_{i}$ 's satisfy $(2.6)$.

In the noncommutative case it is convenient to introduce another set of translation operators $U_{i}$ which satisfy

$$
U_{i} U_{j}=e^{2 \pi i \Theta_{i j}} U_{j} U_{i}
$$


The $U_{i}$ 's generate the algebra of functions on a quantum torus. We denote this algebra, $\mathcal{A}_{\Theta}$. Also note that the $\mathcal{U}_{i}$ 's are the generators of $\mathcal{A}_{(-\Theta)}$. An expanded discussion of this and other issues in noncommutative geometry can be found in $[66,67]$.

This algebra can be realized as a subalgebra of the quantum plane algebra, which is generated by $\sigma_{i}$ satisfying

$$
\left[\sigma_{i}, \sigma_{j}\right]=-2 \pi i \Theta_{i j}
$$

Then one can realize the generators of $\mathcal{A}_{\Theta}$ as

$$
U_{i} \stackrel{\text { def }}{=} e^{i \sigma_{i}}
$$

To realize the $\mathcal{U}_{i}$ generators we also introduce partial derivatives satisfying ${ }^{2}$

$$
\left[\partial^{i}, \sigma_{j}\right]=\delta_{j}^{i},\left[\partial^{i}, \partial^{j}\right]=0
$$

Now one can write the $\mathcal{U}_{i}$ generators as

$$
\mathcal{U}_{i}=e^{i \sigma_{i}-2 \pi \Theta_{i j} \partial^{j}}
$$

Note that both $\sigma_{i}$ and $\partial^{i}$ act as translation generators on the $\sigma_{i}$ 's, and the exponent in the $\mathcal{U}_{i}$ 's is just the linear combination that commutes with all the $\sigma_{i}{ }^{\prime}$ s. Thus

$$
\left[\mathcal{U}_{i}, U_{j}\right]=0
$$

For vanishing $\Theta$ one sees that $U_{i}$ and $\mathcal{U}_{i}$ coincide.

\footnotetext{
${ }^{2}$ Just as in the classical case, one can also introduce quantum exterior forms $d \sigma_{i}$, which anti-commute with each other and commute with all other variables.
} 
The simplest example of solutions of the quotient conditions (2.2) are quantum connections on trivial bundles

$$
X^{j}=-2 \pi i D^{j}=-2 \pi i\left(\partial^{j}-i A^{j}\left(U_{k}\right)\right)
$$

In the noncommutative case the matrix elements of $A^{j}$ are elements of $\mathcal{A}_{\Theta}$. Again using the representation (2.12) of $X^{i}$ in the Matrix model action one obtains a NCSYM action [49]. However we postpone writing this action until we study more general solutions which are connections on nontrivial bundles.

For the commutative case, matrix compactification on $T^{d}$ results in a SYM gauge theory in $d+1$ dimensions on the dual torus. In the limit when the size of the original torus vanishes the dual torus becomes $\mathbf{R}^{d}$, therefore one obtains the opposite of dimensional reduction. If one starts from a Euclidean 10-dimensional SYM and dimensionally reduces in all directions including the Euclidean time one obtains the IKKT [65] functional. Matrix compactification of one direction in the IKKT functional results in the finite temperature action of the original theory (2.3).

\subsection{Twisted Quantum Bundles on $T^{2}$}

There exist more general solutions of the quotient conditions (2.2) which are connections on twisted bundles. They correspond to compactification of the DLCQ of M-theory in the presence of transversely wrapped membranes. Again the solution is a sum of two terms, a constant curvature connection $\nabla^{i}$ and a fluctuating 
part

$$
\begin{aligned}
X^{i} & =-2 \pi i\left(\nabla^{i}-i A^{i}\left(Z_{j}\right)\right), \\
X^{a} & =X^{a}\left(Z_{i}\right), \\
\psi & =\psi\left(Z_{i}\right) .
\end{aligned}
$$

Here the $Z_{i}$ 's are $n \times n$ matrices with operator entries and, just like the $\widetilde{U}_{i}$ 's for the trivial bundle, commute with the $U_{i}$ 's, but now are sections of the twisted bundle whose exact form will be discussed shortly. However, while for the trivial bundle $A^{i}, X^{a}$ and the spinorial components of $\psi$ are $n \times n$ matrix functions, in (2.13) $A^{i}, X^{a}$ and the components of $\psi$ are one-dimensional functions but with matrix arguments. Later, this will allow us to establish a relationship between a SYM on a twisted $U(n)$ bundle and one on a $U(1)$ bundle.

In this chapter we concentrate for simplicity on the two dimensional case. Following [36], up to a gauge transformation the constant curvature connection can be written as

$$
\nabla^{1}=\partial^{1}, \nabla^{2}=\partial^{2}-i f \sigma_{1},
$$

where $f$ is the constant field strength

$$
\left[\nabla^{1}, \nabla^{2}\right]=-i f
$$

Such a gauge field can only exist in a non-trivial bundle. One can introduce transition functions $\Omega_{i}$ such that the sections of the fundamental bundle satisfy the twisted boundary conditions

$$
\Phi\left(\sigma_{1}+2 \pi, \sigma_{2}\right)=\Omega_{1}\left(\sigma_{1}, \sigma_{2}\right) \Phi\left(\sigma_{1}, \sigma_{2}\right)
$$




$$
\Phi\left(\sigma_{1}, \sigma_{2}+2 \pi\right)=\Omega_{2}\left(\sigma_{1}, \sigma_{2}\right) \Phi\left(\sigma_{1}, \sigma_{2}\right)
$$

Similarly the adjoint sections satisfy

$$
\begin{aligned}
& \Psi\left(\sigma_{1}+2 \pi, \sigma_{2}\right)=\Omega_{1}\left(\sigma_{1}, \sigma_{2}\right) \Psi\left(\sigma_{1}, \sigma_{2}\right) \Omega_{1}\left(\sigma_{1}, \sigma_{2}\right)^{-1}, \\
& \Psi\left(\sigma_{1}, \sigma_{2}+2 \pi\right)=\Omega_{2}\left(\sigma_{1}, \sigma_{2}\right) \Psi\left(\sigma_{1}, \sigma_{2}\right) \Omega_{2}\left(\sigma_{1}, \sigma_{2}\right)^{-1} .
\end{aligned}
$$

Consistency of the transition functions of the bundle requires that

$$
\Omega_{1}\left(\sigma_{1}, \sigma_{2}+2 \pi\right) \Omega_{2}\left(\sigma_{1}, \sigma_{2}\right)=\Omega_{2}\left(\sigma_{1}+2 \pi, \sigma_{2}\right) \Omega_{1}\left(\sigma_{1}, \sigma_{2}\right) .
$$

This relation is known in the mathematical literature as the cocycle condition. The covariant derivatives transform just as the adjoint sections

$$
\begin{aligned}
& \nabla^{i}\left(\sigma_{1}+2 \pi, \sigma_{2}\right)=\Omega_{1}\left(\sigma_{1}, \sigma_{2}\right) \nabla^{i}\left(\sigma_{1}, \sigma_{2}\right) \Omega_{1}\left(\sigma_{1}, \sigma_{2}\right)^{-1}, \\
& \nabla^{i}\left(\sigma_{1}, \sigma_{2}+2 \pi\right)=\Omega_{2}\left(\sigma_{1}, \sigma_{2}\right) \nabla^{i}\left(\sigma_{1}, \sigma_{2}\right) \Omega_{2}\left(\sigma_{1}, \sigma_{2}\right)^{-1} .
\end{aligned}
$$

A particular solution for the transition functions compatible with the constant curvature connection (2.14) and satisfying the cocycle condition is given by

$$
\Omega_{1}=e^{i m \sigma_{2} / n} U, \Omega_{2}=V
$$

where $U, V$ are $n \times n$ unitary matrices satisfying

$$
U V=e^{-2 \pi i m / n} V U
$$

Using the representation given in [36] one has

$$
U_{k l}=e^{2 \pi i k m / n} \delta_{k, l}, \quad V_{k l}=\delta_{k+1, l},
$$


where the subscripts are identified with period $n$.

One can express the above matrices in terms of the standard 't Hooft matrices [68] denoted here by $U^{\prime}$ and $V^{\prime}$ and satisfying

$$
U^{\prime} V^{\prime}=e^{-2 \pi i / n} V^{\prime} U^{\prime}, \quad U^{\prime n}=V^{\prime n}=1 .
$$

The relation is given by

$$
U=e^{2 \pi i m / n} U^{\prime m}, \quad V=V^{\prime}
$$

The phase in (2.19) is due to the nonstandard definition of $U$ used in [36]. This has certain advantages but similar phases will appear when comparing the results of [36] with similar results where the standard 't Hooft matrices were used. We also introduce a unitary matrix $K$ which changes the representation so that $V^{\prime}$ is diagonal, and satisfies

$$
K U^{\prime} K^{-1}=V^{\prime-1}, \quad K V^{\prime} K^{-1}=U^{\prime}
$$

Note that $n$ is quantized since one is considering a $U(n)$ gauge theory and $m$ is quantized since the magnetic flux $f$ through $T_{2}$ is quantized

$$
2 \pi f=\frac{m}{n+m \theta}
$$

where $\theta=\Theta_{12}$. In M-theory $m$ is the transversal membrane wrapping number.

One can solve the boundary conditions (2.15) for the fundamental sections as in [36] generalizing a previous result for $m=1$ in the commutative case presented in [26]. Using the ordered exponential explained below, the general solution has 
the form

$$
\Phi_{k}\left(\sigma_{1}, \sigma_{2}\right)=\sum_{s \in \mathbf{Z}} \sum_{j=1}^{m} E\left(\frac{m}{n}\left(\frac{\sigma_{2}}{2 \pi}+k+n s\right)+j, i \sigma_{1}\right) \widehat{\phi}_{j}\left(\frac{\sigma_{2}}{2 \pi}+k+n s+\frac{n j}{m}\right) .
$$

The ordered exponential [36] is defined for two variables whose commutator is a $c$-number

$$
E(A, B)=\frac{1}{1-[A, B]} \sum_{l=0}^{\infty} \frac{1}{l !} A^{l} B^{l}
$$

The normalization is such that

$$
E(-B, A) E(A, B)=1
$$

and it has the following desirable properties similar to the usual exponential

$$
\begin{aligned}
& E(A+c, B)=E(A, B) e^{c B}, \\
& E(A, B+c)=e^{c A} E(A, B) .
\end{aligned}
$$

The $\widehat{\phi}_{j}$ functions are defined on the whole real axis and are unrestricted except for the behavior at infinity. They should be considered as vectors in a Hilbert space on which all the elements of the algebra are represented.

Next we explain in some detail how to obtain this result. First define

$$
\phi\left(\sigma_{1}, \sigma_{2}\right) \stackrel{\text { def }}{=} \Phi_{k}\left(\sigma_{1}, \sigma_{2}-2 \pi(k-1)\right) .
$$

The second boundary condition (2.15) implies that the definition of $\phi$ is consistent, i.e. $k$-independent. Using $V^{n}=1$ one also finds that $\phi$ is a periodic function in $\sigma_{2}$

$$
\phi\left(\sigma_{1}, \sigma_{2}+2 \pi n\right)=\phi\left(\sigma_{1}, \sigma_{2}\right)
$$


The other boundary condition gives

$$
\phi\left(\sigma_{1}+2 \pi, \sigma_{2}\right)=e^{i m\left(\sigma_{2}+2 \pi\right) / n} \phi\left(\sigma_{1}, \sigma_{2}\right) .
$$

It is convenient to separate out a factor to eliminate the above twist

$$
\phi\left(\sigma_{1}, \sigma_{2}\right)=f\left(\sigma_{1}, \sigma_{2}\right) \check{\phi}\left(\sigma_{1}, \sigma_{2}\right)
$$

and to require a simpler periodicity condition for $\check{\phi}$

$$
\check{\phi}\left(\sigma_{1}+2 \pi, \sigma_{2}\right)=\check{\phi}\left(\sigma_{1}, \sigma_{2}\right)
$$

Then the function $f$ must satisfy

$$
f\left(\sigma_{1}+2 \pi, \sigma_{2}\right)=e^{i m\left(\sigma_{2}+2 \pi\right) / n} f\left(\sigma_{1}, \sigma_{2}\right)
$$

This is satisfied exactly for

$$
f\left(\sigma_{1}, \sigma_{2}\right)=E\left(\frac{m}{n}\left(\frac{\sigma_{2}}{2 \pi}+1\right), i \sigma_{1}\right)
$$

where the ordered exponential defined above was used in the right hand side. One can Fourier transform $\check{\phi}$ in $\sigma_{1}$

$$
\check{\phi}\left(\sigma_{1}, \sigma_{2}\right)=\sum_{p \in \mathbf{Z}} e^{i p \sigma_{1}} \phi_{p}\left(\sigma_{2}\right)
$$

and using the property (2.21) of the ordered exponential one obtains

$$
\phi\left(\sigma_{1}, \sigma_{2}\right)=\sum_{p \in \mathbf{Z}} E\left(\frac{m}{n}\left(\frac{\sigma_{2}}{2 \pi}+1\right)+p, i \sigma_{1}\right) \phi_{p}\left(\sigma_{2}\right) .
$$

Let $p=m s+j$ with $j=1, \ldots, m$ and $s$ is an integer. Then the solution can be written as

$$
\phi\left(\sigma_{1}, \sigma_{2}\right)=\sum_{s \in \mathbf{Z}} \sum_{j=1}^{m} E\left(\frac{m}{n}\left(\frac{\sigma_{2}}{2 \pi}+1\right)+m s+j, i \sigma_{1}\right) \phi_{s, j}\left(\sigma_{2}\right),
$$


where $\phi_{s, j} \stackrel{\text { def }}{=} \phi_{m s+j}$. Periodicity in $\sigma_{2}$ then implies $\phi_{s-1, j}\left(\sigma_{2}+2 \pi n\right)=\phi_{s, j}\left(\sigma_{2}\right)$ so that using this recursively one has $\phi_{s, j}\left(\sigma_{2}\right)=\phi_{0, j}\left(\sigma_{2}+2 \pi n s\right)$. Finally after defining $\widetilde{\phi}_{j}(x) \stackrel{\text { def }}{=} \phi_{0, j}(2 \pi(x-1))$ one obtains

$$
\Phi_{k}\left(\sigma_{1}, \sigma_{2}\right)=\sum_{s \in \mathbf{Z}} \sum_{j=1}^{m} E\left(\frac{m}{n}\left(\frac{\sigma_{2}}{2 \pi}+k+n s\right)+j, i \sigma_{1}\right) \widetilde{\phi}_{j}\left(\frac{\sigma_{2}}{2 \pi}+k+n s\right) .
$$

This is the result mentioned above up to another redefinition

$$
\widetilde{\phi}_{j}(x)=\widehat{\phi}_{j}\left(x+\frac{n}{m} j\right)
$$

While the solutions for the sections of the fundamental bundle given in [36] are suitable for showing the equivalence to the projective modules of [27] as will be discussed in Section 3.3, the appearance of the ordered exponential is somewhat inconvenient. Using the special form of the transition functions one can rewrite the solution in an equivalent but simpler form. The transition functions in this gauge do not contain $\sigma_{1}$ and it is convenient to order all $\sigma_{1}$ to the right in the solution. Using $V^{n}=1$ in the second condition (2.15) one can express all $n$ components of $\Phi$ in terms of a single function with period $2 \pi n$ in $\sigma_{2}$. After Fourier transforming in $\sigma_{2}$ and imposing both boundary conditions (2.15) one obtains the general solution

$$
\Phi_{k}\left(\sigma_{1}, \sigma_{2}\right)=\sum_{p \in \mathbf{Z}} e^{2 \pi i\left(\sigma_{2} / 2 \pi+k\right) p / n} e^{2 \pi i\left(\sigma_{1} / 2 \pi-p / m\right) m / n} \widehat{\varphi}_{p}\left(\sigma_{1} / 2 \pi-p / m\right)
$$

where only $m$ of the $\widehat{\varphi}_{p}$ functions are independent, since

$$
\widehat{\varphi}_{p+m}(x)=\widehat{\varphi}_{p}(x) .
$$

Using the same technique one can show that an arbitrary adjoint section has 
the following expansion

$$
\Psi\left(\sigma_{1}, \sigma_{2}\right)=\sum_{s, t \in \mathbf{Z}} c_{s t} Z_{1}^{s} Z_{2}^{-t}
$$

Here $c_{s, t}$ are $c$-numbers and

$$
Z_{1}=e^{i \sigma_{1} /(n+m \theta)} V^{b}, \quad Z_{2}=e^{i \sigma_{2} / n} U^{-b},
$$

where $b$ is an integer, such that one can find another integer $a$ satisfying $a n-b m=$ 1. For $n$ and $m$ relatively prime one can always find integer solutions to this equation. Again, let me emphasize that the $Z_{i}$ 's commute with the $U_{i}$ 's. They are generators of the algebra of functions on a new quantum torus

$$
Z_{1} Z_{2}=e^{2 \pi i \theta^{\prime}} Z_{2} Z_{1}
$$

where $\theta^{\prime}$ is obtained by an $S L(2, \mathbf{Z})$ fractional transformation from $\theta$

$$
\theta^{\prime}=\frac{a \theta+b}{m \theta+n}
$$

Now we outline how to obtain this result. First note that

$$
\Psi\left(\sigma_{1}+2 \pi n, \sigma_{2}\right)=\Omega_{1}^{n} \Psi\left(\sigma_{1}, \sigma_{2}\right) \Omega_{1}^{-n}=\Psi\left(\sigma_{1}-2 \pi \theta m, \sigma_{2}\right)
$$

In the last equality we used the fact that $U^{n}=1$, and the exponential formula to shift $\sigma_{1}$. Using both boundary conditions one has

$$
\begin{gathered}
\Psi\left(\sigma_{1}+2 \pi(n+m \theta), \sigma_{2}\right)=\Psi\left(\sigma_{1}, \sigma_{2}\right), \\
\Psi\left(\sigma_{1}, \sigma_{2}+2 \pi n\right)=\Psi\left(\sigma_{1}, \sigma_{2}\right) .
\end{gathered}
$$


Next, expand the section as

$$
\Psi\left(\sigma_{1}, \sigma_{2}\right)=\sum_{s, t \in \mathbf{Z}} e^{i s \sigma_{1} /(n+m \theta)} e^{-i t \sigma_{2} / n} \Psi_{s, t},
$$

where $\Psi_{s, t}$ is a $n \times n$ matrix and can be expanded as

$$
\Psi_{s, t}=\sum_{i=i_{0}}^{n+i_{0}} \sum_{j=j_{0}}^{n+j_{0}} c_{s, t, i, j} V^{\prime i} U^{\prime j}
$$

Here $i_{0}, j_{0}$ are two arbitrary integers, allowing us to freely shift the summation limits assuming that $c_{s, t, i+n, j}=c_{s, t, i, j+n}=c_{s, t, i, j}$. Then one can obtain further restrictions on the $c_{s, t, i, j}$ coefficients using the boundary conditions (2.16). For example using the first equation (2.16) and comparing like coefficients in the Fourier expansion one has

$$
c_{s, t, i, j} e^{2 \pi i s /(n+m \theta)}=c_{s, t, i, j} e^{-2 \pi i m i / n} e^{-2 \pi i s m \theta /[n(n+\theta)]} .
$$

From this and the similar relation obtained by imposing the second equation (2.16) one sees that $c_{s, t, i, j}$ vanish unless $(s+m i) / n=k$ and $(t+j) / n=s$ for $k$ and $s$ two integers. These equations have multiple solutions. However, if $(i, j)$ and $\left(i^{\prime}, j^{\prime}\right)$ are two solutions then $i-i^{\prime} \in n \mathbf{Z}$ and $j-j^{\prime} \in n \mathbf{Z}$. This ensures that only one term survives in the sum (2.23) over $i$ and $j$. Choosing for later convenience $i_{0}=s b$ and $j_{0}=m b t$ one has

$$
\Psi\left(\sigma_{1}, \sigma_{2}\right)=\sum_{s, t \in \mathbf{Z}} e^{i s \sigma_{1} /(n+m \theta)} e^{-i t \sigma_{2} / n} \sum_{i=s b}^{n+s b} \sum_{j=m b t}^{n+m b t} c_{s, t, i, j} V^{\prime i} U^{\prime j} .
$$

Since $n$ and $m$ are relatively prime let $a, b \in \mathbf{Z}$ such that $a n-b m=1$. Then

$$
k=a s, l=a t, i=b s, j=m b t
$$


is an integer solution inside the $i$ and $j$ summation range. Dropping the $i, j$ indices since they are determined by $s$ and $t$ one has

$$
\Psi\left(\sigma_{1}, \sigma_{2}\right)=\sum_{s, t \in \mathbf{Z}} c_{s, t}\left(e^{i \sigma_{1} /(n+m \theta)} V^{\prime b}\right)^{s}\left(e^{i \sigma_{2} / n} U^{\prime-m b}\right)^{-t}
$$

which is just (2.22) after an additional phase redefinition of $c_{s, t}$ to accommodate the phase difference between $U$ and $U^{\prime m}$.

\subsection{Twisted Quantum Bundles on Tori}

In this section we construct quantum $U(n)$ bundles on $d$-dimensional noncommutative tori which admit constant curvature connections with vanishing $s u(n)$ curvature. This is done by finding explicit transition functions compatible with such a connection. We employ a method which is a straightforward generalization

of $[36,40]$. Using a gauge transformation the constant curvature connection can be brought into the form

$$
\nabla^{i}=\partial^{i}+i F^{i j} \sigma_{j}
$$

where $F$ is an antisymmetric matrix. This differs from the gauge used in the previous section, but is very convenient for the higher dimensional cases. From now on this gauge will be used throughout the thesis unless otherwise stated. Define the constant curvature to be

$$
\mathcal{F}_{(0)}^{j k}=i\left[\nabla^{j}, \nabla^{k}\right]
$$

Then, using the commutation relations (2.11) one can calculate

$$
\mathcal{F}_{(0)}=(2 F+2 \pi F \Theta F)
$$


In general, such a connection can only exist on a non-trivial bundle. One can introduce transition functions $\Omega_{i}$ such that the connection satisfies the twisted boundary conditions

$$
\nabla^{i}\left(\sigma_{m}+2 \pi \delta_{m}^{j}\right)=\Omega_{j}\left(\sigma_{m}\right) \nabla^{i}\left(\sigma_{m}\right) \Omega_{j}^{-1}\left(\sigma_{m}\right) .
$$

One can try to find solutions for the transition functions of the form

$$
\Omega_{j}=e^{i P^{j l} \sigma_{l}} W_{j},
$$

where $P$ is an arbitrary constant $d$-dimensional matrix and the $W_{i}$ 's are constant, unitary $n$-dimensional matrices. The boundary conditions (2.25) imply the following equivalent relations

$$
\begin{gathered}
P=(1+2 \pi F \Theta)^{-1} 2 \pi F=2 \pi F(1+\Theta 2 \pi F)^{-1}, \\
2 \pi F=P(1-\Theta P)^{-1}=(1-P \Theta)^{-1} P .
\end{gathered}
$$

Note that $P$ must be antisymmetric because of our gauge choice. Consistency of the transition functions of the bundle is the cocycle condition

$$
\Omega_{j}\left(\sigma_{m}+2 \pi \delta_{m}^{i}\right) \Omega_{i}\left(\sigma_{m}\right)=\Omega_{i}\left(\sigma_{m}+2 \pi \delta_{m}^{j}\right) \Omega_{j}\left(\sigma_{m}\right) .
$$

In our case it implies

$$
W_{i} W_{j}=e^{-2 \pi i M^{i j} / n} W_{j} W_{i},
$$

where the antisymmetric matrix $M$ is given by

$$
M=n(2 P-P \Theta P) .
$$


By taking the determinant of both sides of (2.27) one finds that $M$ must have integer entries. In the classical case $M^{i j}$ corresponds to the value of the first Chern class on the $(i j)$ two-cycle of the torus. In the auxiliary Type IIA string theory, $M$ is interpreted as D2 brane winding. This interpretation remains true in the quantum case.

Let $q$ be the greatest common divisor of $n$ and the nonvanishing entries of $M$

$$
q=\operatorname{gcd}\left(n, M^{i j}\right)
$$

Next one defines $\tilde{n}$ and $\widetilde{M}$ which have relatively prime entries

$$
n=q \tilde{n}, \quad M=q \widetilde{M} .
$$

It is convenient to consider $W_{i}$ 's which have block diagonal form with $q$ identical blocks along the diagonal

$$
W_{i}=\left(\begin{array}{ccc}
\widetilde{W}_{i} & & \\
& \ddots & \\
& & \widetilde{W}_{i}
\end{array}\right) .
$$

Here $\widetilde{W}_{i}$ are $\tilde{n}$-dimensional matrices. Alternatively one can write this in tensor product notation

$$
W_{i}=I_{q} \otimes \widetilde{W}_{i}
$$

The transition functions are also block diagonal and can be written

$$
\Omega_{i}=I_{q} \otimes \omega_{i}
$$

To find explicit boundary conditions, following 't Hooft [69], one makes the ansatz

$$
\widetilde{W}_{i}=U^{a^{i}} V^{b^{i}}
$$


where $a^{i}$ and $b^{i}$ are integers and $U$ and $V$ are the clock and shift matrices $[68,69]$

$$
U_{k l}=e^{2 \pi i(k-1) / \tilde{n}} \delta_{k, l}, \quad V_{k l}=\delta_{k+1, l}, \quad k, l=1, \ldots, \tilde{n},
$$

and the subscripts are identified with period $\tilde{n}$. They satisfy

$$
U V=e^{-2 \pi i / \tilde{n}} V U
$$

Then (2.27) leads to the following relation

$$
\widetilde{M}^{i j}=\left(a^{i} b^{j}-b^{i} a^{j}\right) \bmod (\tilde{n})
$$

For two or three dimensional tori, one can find integers $a^{i}$ and $b^{i}$ such that $(2.30)$ holds for arbitrary $M$, as will be shown in Section 2.5. In higher dimensional cases the ansatz is not sufficiently general to describe arbitrary bundles. In particular, one can always perform a change of lattice basis such that the only nonvanishing

components of $M$ are $M^{d-1, d}=-M^{d, d-1}$, while in general, an arbitrary antisymmetric matrix can not be brought into such a form. Furthermore, for $d>3$, even in the commutative case, generic bundles do not admit connections with vanishing $s u(n)$ constant curvature. A more general construction could be obtained by allowing for an arbitrary constant curvature connection.

\subsection{Adjoint Sections on Twisted Bundles}

In this section we analyze the structure of adjoint sections on twisted bundles. The scalar and fermion fields are examples of such sections. We also write the 
connection as a sum of a constant curvature connection $\nabla^{i}$, and a fluctuating part $A^{i}$

$$
D^{i}=\nabla^{i}-i A^{i}
$$

Note that $A^{i}$ is also an adjoint section. Since it is the difference between two connections it transforms covariantly under gauge transformations. It should not be confused with a gauge potential. Adjoint sections are $n$-dimensional matrices with entries which are elements of the quantum plane algebra (2.11) and obey the twisted boundary conditions

$$
\Psi\left(\sigma_{i}+2 \pi \delta_{i}^{j}\right)=\Omega_{j} \Psi\left(\sigma_{i}\right) \Omega_{j}^{-1}
$$

Next we try to find the general solution of (2.31) and write it in unconstrained form, reflecting the global properties of the bundle. First consider the simpler example of a $U(n)$ NCSYM on a trivial bundle over a two-torus. Since $\Omega_{i}=1$ one has

$$
\Psi=\sum_{a, b=1}^{n} E^{a b} \otimes\left[\sum_{i_{1} i_{2} \in \mathbf{Z}} \Psi_{i_{1} i_{2}}^{a b} U_{1}^{i_{1}} U_{2}^{i_{2}}\right]
$$

where $E^{a b}$ are $n$-dimensional matrices with one nonzero entry, $\left(E^{a b}\right)_{i j}=\delta_{i}^{a} \delta_{j}^{b}$, and $\Psi_{i_{1} i_{2}}^{a b}$ are $c$-numbers. In other words, each matrix element of the adjoint section is an arbitrary function on the quantum torus. For a twisted $U(n)$ bundle with magnetic flux $m$, such that $n$ and $m$ are relatively prime, one can show $[27,36,40]$ that the adjoint sections have the expansion

$$
\Psi=\sum_{i_{1} i_{2} \in \mathbf{Z}} \Psi_{i_{1} i_{2}} Z_{1}^{i_{1}} Z_{2}^{i_{2}}
$$


where now the coefficients $\Psi_{i_{1} i_{2}}$ are $c$-numbers, and $Z_{i}$ are $n$-dimensional matrices with noncommutative entries satisfying

$$
Z_{1} Z_{2}=e^{2 \pi i \theta^{\prime}} Z_{2} Z_{1}
$$

Thus the $Z_{i}$ 's satisfy the commutation relations of a generators of the quantum torus. This shows that the set of sections is isomorphic to the set of functions on a dual torus, and is very similar to the set of adjoint sections of a $U(1)$ NCSYM theory. For two and three dimensional adjoint bundles with arbitrary magnetic fluxes, we will show that the general solution takes the form

$$
\Psi=\sum_{a b=1}^{q} E^{a b} \otimes\left[\sum_{i_{1} i_{2} \ldots i_{d} \in \mathbf{Z}} \Psi_{i_{1} i_{2} \ldots i_{d}}^{a b} Z_{1}^{i_{1}} Z_{2}^{i_{2}} \ldots Z_{d}^{i_{d}}\right], \quad d=2,3 .
$$

Here $E^{a b}$ are $q$ dimensional.

Begin by writing $\Psi$ in tensor notation

$$
\Psi\left(\sigma_{i}\right)=\sum_{a, b=1}^{q} E^{a b} \otimes \Psi^{a b}\left(\sigma_{i}\right)
$$

where $\Psi^{a b}\left(\sigma_{i}\right)$ are $\tilde{n}$-dimensional matrices with noncommutative entries. Imposing the boundary conditions (2.31) and using (2.28) one obtains

$$
\Psi^{a b}\left(\sigma_{i}+2 \pi \delta_{i}^{j}\right)=\omega_{j} \Psi^{a b}\left(\sigma_{i}\right) \omega_{j}^{-1}
$$

A less restrictive but very convenient constraint is obtained by shifting $\sigma_{i}$ by $2 \pi \tilde{n}$ using (2.33)

$$
\Psi^{a b}\left(\sigma_{i}+2 \pi \tilde{n} \delta_{i}^{j}\right)=\omega_{j}^{\tilde{n}} \Psi^{a b}\left(\sigma_{i}\right) \omega_{j}^{-\tilde{n}}
$$

In (2.34) all the matrix factors disappear since $U^{\tilde{n}}=V^{\tilde{n}}=1$. The $\sigma_{i}$ dependent exponential of (2.26) survives and acts like a translation operator due to the 
commutation relations (2.11). This implies the following periodicity relation

$$
\Psi^{a b}\left(\sigma_{i}+2 \pi \tilde{n}\left(Q^{-1}\right)_{i}^{j}\right)=\Psi^{a b}\left(\sigma_{i}\right)
$$

where

$$
Q^{-1}=1-P \Theta
$$

Next we try to find solutions of the form

$$
Z_{i}=e^{i \sigma_{j} Q_{k}^{j} N_{i}^{k} / \tilde{n}} U^{s_{i}} V^{t_{i}}, \quad i=1 \ldots d
$$

Here $s_{j}$ and $t_{j}$ are integers and the exponent was chosen so that it is compatible with the constraint (2.35) if the matrix $N$ has integer entries. One can show that $Z_{i}$ is compatible with the boundary conditions $(2.33)$ if

$$
N_{j}^{i}=\left(b^{i} s_{j}-a^{i} t_{j}\right) \bmod (\tilde{n})
$$

where $a^{i}$ and $b^{i}$ are defined by (2.30). In the next two sections we will consider in detail the two and three dimensional cases, and find $a^{i}, b^{i}, s_{j}$ and $t_{j}$ such that (2.30) and (2.37) hold. Furthermore, for properly chosen integers $a^{i}, b^{i}, s_{j}$ and $t_{j}$, one can show that an arbitrary adjoint section can be expanded in terms of the $Z_{i}$ 's as in (2.32). For a proof of this statement in two dimensions see [40]. It is convenient to define another matrix which will be used shortly,

$$
L_{i j}=\left(s_{i} t_{j}-t_{i} s_{j}\right) \bmod (\tilde{n}) .
$$

In the remainder of this section we calculate the commutation relations satisfied by the $Z_{i}$ 's and the constant curvature connection (2.24). Using their explicit 
form (2.36) one finds, after some matrix algebra,

$$
Z_{i} Z_{j}=e^{2 \pi i \Theta_{i j}^{\prime}} Z_{j} Z_{i}
$$

where

$$
\Theta^{\prime}=\tilde{n}^{-2} N^{T} Q^{T} \Theta Q N-\tilde{n}^{-1} L
$$

From (2.39) one can see that the algebra generated by the $Z_{i}$ 's is the algebra of functions on the quantum torus with deformation parameters given by $\Theta^{\prime}$. After some further matrix algebra and using the following identities,

$$
\begin{aligned}
Q & =1+2 \pi F \Theta, \\
Q^{2} & =1+2 \pi \mathcal{F}_{(0)} \Theta=(1-\widetilde{M} \Theta / \widetilde{n})^{-1}, \\
Q^{T} \Theta & =\Theta Q,
\end{aligned}
$$

one can rewrite $\Theta^{\prime}$ as a fractional transformation

$$
\Theta^{\prime}=\Lambda(\Theta) \stackrel{\text { def }}{=}(\mathcal{A} \Theta+\mathcal{B})(\mathcal{C} \Theta+\mathcal{D})^{-1}
$$

Here

$$
\Lambda=\left(\begin{array}{ll}
\mathcal{A} & \mathcal{B} \\
\mathcal{C} & \mathcal{D}
\end{array}\right)
$$

and the $d$-dimensional block matrices are given by

$$
\mathcal{A}=\tilde{n}^{-1}\left(N^{T}+L N^{-1} \widetilde{M}\right), \mathcal{B}=-L N^{-1}, \mathcal{C}=-N^{-1} \widetilde{M}, \mathcal{D}=\tilde{n} N^{-1}
$$

One can check that

$$
\mathcal{A}^{T} \mathcal{D}+\mathcal{C}^{T} \mathcal{B}=1, \mathcal{A}^{T} \mathcal{C}+\mathcal{C}^{T} \mathcal{A}=0, \mathcal{B}^{T} \mathcal{D}+\mathcal{D}^{T} \mathcal{B}=0
$$


and thus $\Lambda$ is an element of $O(d, d \mid \mathbf{R})$, i.e. it satisfies

$$
\Lambda^{T} J \Lambda=J
$$

where

$$
J=\left(\begin{array}{cc}
0 & I_{d} \\
I_{d} & 0
\end{array}\right)
$$

In the two and three dimensional examples that will be discussed later, $\Lambda$ is in fact an element of $S O(d, d \mid \mathbf{Z})$. This is the subgroup with determinant one and integer valued entries in the basis where the metric is given by (2.45). The Weyl spinor representations of $S O(d, d \mid \mathbf{Z})$ are also integral, that is the representation matrices have integer entries. This statement, which is implicit in papers discussing Tduality of Type II string theory, will be proven in the Appendix A. Since the spinor representation of $S O(d, d \mid \mathbf{Z})$ will be used extensively in the following sections, recall that the vector and spinor representations are related by

$$
\mathcal{S}^{-1} \gamma_{s} \mathcal{S}=\Lambda_{s}^{p} \gamma_{p}
$$

and the gamma matrices satisfy

$$
\left\{\gamma_{s}, \gamma_{p}\right\}=2 J_{s p}
$$

Finally, one can show by direct calculation that the commutation relations of the constant curvature connection and $Z_{i}$ have the form

$$
\left[\nabla^{i}, Z_{j}\right]=i H_{j}^{i} Z_{j},
$$


where there is no sum over $j$ and $H=(\tilde{n}-\widetilde{M} \Theta)^{-1} N$. Note that $H$ can also be written in terms of $\Theta$ and some of the block components of $\Lambda$

$$
H^{-1}=\mathcal{C} \Theta+\mathcal{D}
$$

Finally, the following identities will be useful in later sections

$$
\begin{aligned}
H & =\tilde{n}^{-1} Q^{2} N, \\
\operatorname{det} H & =(q \operatorname{det}(Q) / n)^{2}, \\
\operatorname{det}\left(Q^{2}\right) & =\left(1-\frac{\operatorname{tr}(\widetilde{M} \Theta)}{2 \tilde{n}}\right)^{-2} . \\
M & =2 \pi n Q^{-1} \mathcal{F}_{(0)} Q^{-T}, \\
M^{i j} \varepsilon_{i j l} & =M^{i j} \varepsilon_{i j k} Q_{l}^{k} .
\end{aligned}
$$

Note that with the exception of the last relation all the others are valid for tori of arbitrary dimension provided one works on the bundles discussed in Section 2.3.

\subsection{Two and Three Dimensional Solutions}

Although the twisted two dimensional case has been discussed extensively in the literature $[27,35,36,40]$, we will review it here in a form that readily admits generalization to higher dimensional compactifications. I will then give a complete description of the three dimensional adjoint bundles.

In the two dimensional case the antisymmetric matrices $\Theta$ and $M$ have the form

$$
\Theta=\left(\begin{array}{cc}
0 & \theta \\
-\theta & 0
\end{array}\right), \quad M=\left(\begin{array}{cc}
0 & m \\
-m & 0
\end{array}\right)
$$


where $\theta$ is the deformation parameter and $m$ is the magnetic flux, which is interpreted as the number of D2 branes wrapping the two-torus.

One can verify that the integers

$$
\left(a^{i}\right)=(\tilde{m}, 0),\left(b^{i}\right)=(0,1),
$$

where $n=q \tilde{n}$ and $m=q \tilde{m}$, satisfy (2.30). Then choosing $s_{i}=(0,1)$ and $t_{i}=(b, 0)$, where $b$ is an integer such that $a \tilde{n}-b \tilde{m}=1$, one has $N=I_{2}$. One can now use (2.38) and (2.43) to find

$$
\Lambda=\left(\begin{array}{cc}
a I_{2} & b \varepsilon \\
-\tilde{m} \varepsilon & \tilde{n} I_{2}
\end{array}\right),
$$

where $\varepsilon$ is a two dimensional matrix with the only nonvanishing entries given by $\varepsilon_{12}=-\varepsilon_{21}=1$. Group elements of the form above are in an $S L(2, \mathbf{Z})$ subgroup of $S O(2,2 \mid \mathbf{Z})$. This subgroup is isomorphic with one of the Weyl spinor representations of $S O(2,2 \mid \mathbf{Z})$. This feature is not generic for higher dimensional compactifications and reflects the fact that $S O(2,2 \mid \mathbf{Z}) \sim S L(2, \mathbf{Z}) \times S L(2, \mathbf{Z})$, so that it is not simple.

The algebra of the $Z_{i}^{\prime}$ 's is then determined by $\Theta^{\prime}$ which is given by the fractional transformation (2.41). In two dimensions, the $S O(2,2 \mid \mathbf{Z})$ fractional transformation (2.41) can also be written in the more familiar form, used in $[27,36]$, as a $S L(2, \mathbf{Z})$ fractional transformation acting on $\theta$

$$
\theta^{\prime}=\frac{a \theta+b}{\tilde{m} \theta+\tilde{n}}
$$

One can also check that the other $S L(2, \mathbf{Z})$ subgroup, made of elements of the 
form

$$
\left(\begin{array}{cc}
R & 0 \\
0 & \left(R^{T}\right)^{-1}
\end{array}\right),
$$

acts trivially on $\theta$. This subgroup is generalized to $S L(d, \mathbf{Z})$ in compactifications on a $d$-dimensional torus, and will play in important role later, but only for the two dimensional compactification it leaves $\Theta$ invariant. The $Z_{i}$ 's then obey the following algebra

$$
Z_{1} Z_{2}=e^{2 \pi i \theta^{\prime}} Z_{2} Z_{1}
$$

As will be shown shortly, the rank of the gauge group and the magnetic flux transform in an integral Weyl spinor representation of $S O(2,2 \mid \mathbf{Z})$. Using the creation and annihilation operators introduced in the Appendix A one can write such a spinor as

$$
n|0\rangle+m a_{1}^{\dagger} a_{2}^{\dagger}|0\rangle
$$

Using (2.46) one can show that the spinor representation of (2.51) transforms the above state into $q|0\rangle$. In the Weyl basis one can write the action as

$$
\left(\begin{array}{l}
q \\
0
\end{array}\right)=S\left(\begin{array}{l}
n \\
m
\end{array}\right),
$$

where

$$
S=\left(\begin{array}{cc}
a & -b \\
-\tilde{m} & \tilde{n}
\end{array}\right)
$$

In Section 3.1 we will show, employing the expansion of the adjoint section in terms of the $Z_{i}$ generators $(2.32)$, how to rewrite the original $U(n)$ NCSYM action on a twisted bundle as a $U(q)$ NCSYM action on a trivial quantum bundle over a torus 
with deformation parameter $\Theta^{\prime}$. The $S L(2, \mathbf{Z})$ transformation, which relates the deformation parameters and the spinors (2.53) of these two NCSYM, can then be interpreted as a duality transformation inherited from T-duality of Type II string theory. This can be seen as follows. The rank and the bundle of the NCSYM theory determine the D brane charges in string theory. These charges transform in a chiral spinor representation of the target space duality group [13]. Given $n$ and $m$ with greatest common divisor $q$, one can perform a T-duality transformation which takes the original D brane configuration into $q$ D0 branes.

Of course the metric and antisymmetric tensor also transform under this duality, and in the proper limit, which we will explain in detail later, the antisymmetric tensor $B$ transforms separately by fractional transformation just as in (2.41). Since the parameters $\Theta_{i j}$ of the NCSYM theory are identified with $B_{i j}$, the background expectation value of the NS antisymmetric tensor of the compactified auxiliary string theory, the expected transformation under target space dualities is (2.41).

Next we turn to the three dimensional case which will be solved by first performing an $S L(3, \mathbf{Z})$ transformation $R$ to bring $M$ in canonical form ${ }^{3}$

$$
M=R M^{0} R^{T},
$$

where

$$
M^{0}=\left(\begin{array}{ccc}
0 & 0 & 0 \\
0 & 0 & m \\
0 & -m & 0
\end{array}\right) .
$$

\footnotetext{
${ }^{3}$ It is always possible to bring an antisymmetric matrix in canonical form using $S L(3, \mathbf{R})$ but here one has to do this using an integral matrix.
} 
While it is always possible to find such a transformation, (2.55) does not define it uniquely. We first find the solution corresponding to $M^{0}$, and then obtain the general solution by using such an $R$.

First note that $M^{0}$ corresponds to a background magnetic field with flux only through the (23) plane, which suggests that the solution should closely resemble the two dimensional one. As before,

$$
\left(a_{0}^{i}\right)=(0, \tilde{m}, 0),\left(b_{0}^{i}\right)=(0,0,1)
$$

satisfy (2.30). Similarly if one sets

$$
\left(s_{i}^{0}\right)=(0,0,1),\left(t_{i}^{0}\right)=(0, b, 0),
$$

one can satisfy (2.37) with the $N^{0}$ matrix given by

$$
N^{0}=\left(\begin{array}{ccc}
\tilde{n} & 0 & 0 \\
0 & 1 & 0 \\
0 & 0 & 1
\end{array}\right) .
$$

The diagonal entries of $N^{0}$ divided by $\tilde{n}$ have the interpretation of wave numbers. Thus one can see that twisting the boundary conditions allows for fractional wave numbers in the second and third directions. Using (2.57) one finds

$$
L^{0}=\left(\begin{array}{ccc}
0 & 0 & 0 \\
0 & 0 & -b \\
0 & b & 0
\end{array}\right) .
$$


One can now use (2.43) to find the $S O(3,3 \mid \mathbf{Z})$ matrix

$$
\Lambda^{0}=\left(\begin{array}{cccccc}
1 & 0 & 0 & 0 & 0 & 0 \\
0 & a & 0 & 0 & 0 & b \\
0 & 0 & a & 0 & -b & 0 \\
0 & 0 & 0 & 1 & 0 & 0 \\
0 & 0 & -\tilde{m} & 0 & \tilde{n} & 0 \\
0 & \tilde{m} & 0 & 0 & 0 & \tilde{n}
\end{array}\right) .
$$

Everything so far is just as in the two dimensional case. Note however that in general $\Theta$ will not be in canonical form, that is, it will not have a form similar to $(2.56)$.

Now, write the general solution for an arbitrary $M$ as

$$
\begin{gathered}
a^{i}=R_{j}^{i} a_{0}^{j}, \quad b^{i}=R_{j}^{i} b_{0}^{j}, \\
s_{i}=s_{j}^{0}, \quad t_{i}=t_{j}^{0}, \\
N=R N^{0}, \\
\Lambda=\Lambda^{0}\left(\begin{array}{cc}
R^{T} & 0 \\
0 & R^{-1}
\end{array}\right) .
\end{gathered}
$$

Just as in the two dimensional case one finds, using (2.46), the Weyl spinor representation matrices corresponding to (2.58) and (2.59)

$$
\begin{gathered}
S^{0}=\left(\begin{array}{cccc}
a & -b & 0 & 0 \\
-\tilde{m} & \tilde{n} & 0 & 0 \\
0 & 0 & 1 & 0 \\
0 & 0 & 0 & 1
\end{array}\right), \\
S=S^{0}\left(\begin{array}{cc}
1 & 0 \\
0 & R^{T}
\end{array}\right) .
\end{gathered}
$$


The rank of the group and the magnetic flux matrix $M$ define a state in the Weyl spinor Fock space

$$
n|0\rangle+\frac{1}{2} M^{i j} a_{i}^{\dagger} a_{j}^{\dagger}|0\rangle .
$$

Now one can check that $S$ acts on this spinor as

$$
\left(\begin{array}{l}
q \\
0 \\
0 \\
0
\end{array}\right)=S\left(\begin{array}{c}
n \\
M^{23} \\
M^{31} \\
M^{12}
\end{array}\right) .
$$

It will be convenient to denote the components of the spinor as

$$
\eta=\left(\begin{array}{c}
n \\
M^{23} \\
M^{31} \\
M^{12}
\end{array}\right) .
$$

As will be shown later (2.60) can be used to relate the original theory to a $U(q)$ theory on a trivial bundle. In Appendix A we show that the Weyl spinor representation of $S O(3,3 \mid \mathbf{Z})$ is in fact isomorphic to $S L(4, \mathbf{Z})$. In this case, in the auxiliary Type IIA string theory, the D0 and D2 branes form $q$ bound states, and the transformation above corresponds to a T-duality transformation that maps the original D brane configuration into a $q \mathrm{D} 0$ branes.

\subsection{Noncommutative Super Yang-Mills Action}

After discussing how to perform integration on a noncommutative torus we will be ready to write the noncommutative Super Yang-Mills action. In the classical case the integral is a linear map that associates to a function its zero mode Fourier 
coefficient. Similarly for an element of $\mathcal{A}_{\Theta}$ of the form $a=\sum a_{i_{1} i_{2} \ldots i_{d}} U_{1}^{i_{1}} U_{2}^{i_{2}} \ldots U_{d}^{i_{d}}$ define the integral as

$$
\int d^{d} \sigma a \stackrel{\text { def }}{=}(2 \pi)^{d} a_{00 \ldots 0}
$$

One can check that this definition has all the desirable properties of the classical integral, such as linearity and translation invariance in $\sigma_{i}$. For definiteness, in the remainder of this section we discuss the three dimensional case.

When twisted $U(n)$ theories are considered, it was found in $[27,36]$ that the integral must be normalized in a particular way to find a duality invariant spectrum. The normalization can also be obtained directly as the Jacobian of a change of integration variables. Note that the integrand, which is the trace of an adjoint section, obeys the following periodicity

$$
\operatorname{tr} \Psi\left(\sigma_{i}\right)=\operatorname{tr} \Psi\left(\sigma_{i}+2 \pi\left(Q^{-1}\right)_{i}^{j}\right)
$$

Since $\operatorname{tr} \Psi\left(\sigma_{i}\right)$ does not have periodicity $2 \pi$ in $\sigma_{i}$ it can not be expanded in terms of the $U_{i}$ variables. One can define new variables $\widehat{\sigma}_{i}=\sigma_{j} Q_{k}^{j} R_{i}^{k}$ and $\widehat{U}_{i}=e^{\widehat{\sigma}_{i}}$, where $R$ is an arbitrary $S L(3, \mathbf{Z})$ transformation. In the following sections we take $R$ to be the matrix that brings $M$ into canonical form (2.55). Then

$$
\int d^{3} \sigma \operatorname{tr} \Psi(\sigma)=\int d^{3} \widehat{\sigma}\left|\operatorname{det}\left(Q^{-1}\right)\right| \operatorname{tr} \Psi\left(\widehat{\sigma} Q^{-1}\right)
$$

where $\operatorname{det}\left(Q^{-1}\right)$ is the Jacobian of the coordinate transformation, and the second integral can now be performed as discussed above, since the integrand has an expansion in terms of the $\widehat{U}_{i}$ variables. Using the expansion (2.32) of $\Psi$ one 
obtains

$$
\int d^{3} \sigma \operatorname{tr} \Psi(\sigma)=(2 \pi)^{3} \tilde{n}\left|\operatorname{det}\left(Q^{-1}\right)\right| \sum_{a=1}^{q} \Psi_{000}^{a a}
$$

The Super Yang-Mills action on a noncommutative three-torus is given by

$$
\begin{aligned}
& \mathcal{S}^{U(n)}= \frac{1}{g_{S Y M}^{2}} \int d t \int d^{3} \sigma \sqrt{\operatorname{det}\left(G^{i j}\right)} \operatorname{tr}\left(\frac{1}{2} G_{i j} \mathcal{F}^{0 i} \mathcal{F}^{0 j}-\right. \\
& \frac{1}{4} G_{i j} G_{k l}\left(\mathcal{F}^{i k}-\mathcal{F}_{(0)}^{i k}\right)\left(\mathcal{F}^{j l}-\mathcal{F}_{(0)}^{j l}\right)+ \\
& \frac{1}{2} \sum_{a} \dot{X}^{a} \dot{X}^{a}-\frac{1}{2} \sum_{a} G_{i j}\left[D^{i}, X^{a}\right]\left[D^{j}, X^{a}\right]+ \\
& \\
&\left.\frac{1}{4} \sum_{a, b}\left[X^{a}, X^{b}\right]\left[X^{a}, X^{b}\right]+\text { fermions }\right),
\end{aligned}
$$

where $\mathcal{F}^{i j}=i\left[D^{i}, D^{j}\right]$ and $\mathcal{F}_{0}^{i j}=i\left[\nabla^{i}, \nabla^{j}\right]$. We have subtracted the constant part of the field strength in the second line of equation (2.63). This is equivalent to adding a constant to the Lagrangian, or equivalently to the Hamiltonian, and has the effect of setting the vacuum energy to zero. The noncommutative pure gauge theory action was written first in [49] and the maximally supersymmetric $U(n)$ NCSYM gauge theory action was written in [27, 38].

For the compactification of the auxiliary Type IIA string theory without wrapped D2 branes, the above action can be obtained directly from the Matrix action. One has to show that the trace over infinite dimensional matrices reduces to a finite dimensional trace and an integral. A formal argument for the commutative case was given in [25] and discussed in detail in [70]. The same argument extends to the noncommutative case. A brief argument was given in [27] showing how to extend this construction when there are D2 branes wrapped on the torus 
in the auxiliary Type IIA string theory, corresponding to magnetic fluxes in the NCSYM gauge theory. Here we just make the assumption that the NCSYM action is independent of the D2 brane charges and that adding D2 branes only results in changing the quantum adjoint bundle. We will provide evidence for this in Section 3.2. 


\section{Chapter 3}

\section{Dualities of the Matrix Model from T-Duality of the Type II String}

In Section 3.1, we will start with the $U(n)$ NCSYM action (2.63) on a twisted quantum bundle with magnetic fluxes $M$ and deformation parameter $\Theta$, and show that after a sequence of field redefinitions it can be rewritten as a $U(q)$ NCSYM action on a trivial bundle over a quantum torus with deformation parameter $\Theta^{\prime}$.

Then, in Section 3.2, we will take the small $\alpha^{\prime}$ and small compactification volume limit in the auxiliary Type IIA string theory and obtain the transformation properties of the metric, antisymmetric tensor, and string coupling constant. These are then compared with the transformation in Section 3.1 using the standard relations between String theory and SYM gauge theory.

Section 3.3 shows the relationship between the physical language used in these constructions and the more abstract mathematical language of Connes and Rieffel. The last section contains some gauge equivalent formulations closely resembling standard constructions in the commutative case.

\section{1 $S O(3,3 \mid \mathbf{Z})$ Duality of Super Yang-Mills}


Using the matrix $H$ defined in (2.48), one can make the following constant curvature and field redefinitions

$$
\begin{gathered}
\widehat{\nabla}^{i} \stackrel{\text { def }}{=}\left(H^{-1}\right)_{j}^{i} \nabla^{j}, \widehat{A}^{i} \stackrel{\text { def }}{=}\left(H^{-1}\right)_{j}^{i} A^{j}, \\
\widehat{D}^{i} \stackrel{\text { def }}{=}\left(H^{-1}\right)_{j}^{i} D^{j}, \\
\widehat{\mathcal{F}}^{k l}=\left[\widehat{\nabla}^{k}, \widehat{A}^{l}\right]-\left[\widehat{\nabla}^{l}, \widehat{A}^{k}\right]-i\left[\widehat{A}^{k}, \widehat{A}^{l}\right] .
\end{gathered}
$$

In terms of the new variables, the commutator of the constant curvature connection and the $Z_{i}$ 's takes the simple form,

$$
\left[\widehat{\nabla}^{i}, Z_{j}\right]=i \delta_{j}^{i} Z_{j}
$$

and the curvature can be expressed as

$$
\mathcal{F}^{i j}=\mathcal{F}_{(0)}^{i j}+H_{k}^{i} H_{l}^{j} \widehat{\mathcal{F}}^{k l}
$$

One can now rewrite the action in terms of the hatted variables and perform the change of coordinates $(2.62)$

$$
\begin{aligned}
\mathcal{S}^{U(n)}= & \frac{1}{g^{\prime 2}{ }_{S Y M}} \int d t \int d^{3} \widehat{\sigma} \sqrt{\operatorname{det}\left(G^{\prime i j}\right)} \frac{1}{\tilde{n}} \operatorname{tr}\left(\frac{1}{2} G_{i j}^{\prime} \widehat{\mathcal{F}}^{0 i} \widehat{\mathcal{F}}^{0 j}-\right. \\
& \frac{1}{4} G_{i j}^{\prime} G_{k l}^{\prime} \widehat{\mathcal{F}}^{i k} \widehat{\mathcal{F}}^{j l}+ \\
& \frac{1}{2} \sum_{a} \dot{X}^{a} \dot{X}^{a}-\frac{1}{2} \sum_{a} G_{i j}^{\prime}\left[\widehat{D}^{i}, X^{a}\right]\left[\widehat{D}^{j}, X^{a}\right]+ \\
& \left.\frac{1}{4} \sum_{a, b}\left[X^{a}, X^{b}\right]\left[X^{a}, X^{b}\right]+\text { fermions }\right) .
\end{aligned}
$$

We have introduced a new gauge coupling and metric given by

$$
g_{S Y M}^{\prime 2}=\tilde{n}\left|\operatorname{det}\left(Q^{-1}\right)\right| g_{S Y M}^{2}
$$




$$
G^{i j}=\left(H^{-1}\right)_{k}^{i}\left(H^{-1}\right)_{l}^{j} G^{k l}
$$

and used (2.50) to make these substitutions.

Next we introduce primed variables $\sigma_{i}^{\prime}, U_{i}^{\prime}$ and partial derivatives $\partial^{\prime i}$ satisfying

$$
\begin{gathered}
{\left[\sigma_{i}^{\prime}, \sigma_{j}^{\prime}\right]=-2 \pi i \Theta_{i j}^{\prime},} \\
{\left[\partial^{\prime i}, \sigma_{j}^{\prime}\right]=\delta_{j}^{i}, \quad\left[\partial^{\prime i}, \partial^{\prime j}\right]=0,} \\
U_{i}^{\prime} \stackrel{\text { def }}{=} e^{i \sigma_{i}^{\prime}} \\
U_{i}^{\prime} U_{j}^{\prime}=e^{2 \pi i \Theta_{i j}^{\prime}} U_{j}^{\prime} U_{j}^{\prime} .
\end{gathered}
$$

Comparing the algebra satisfied by $Z_{i}$ and $\widehat{\nabla}^{i}$ on one hand and $U_{i}^{\prime}$ and $\partial_{i}^{\prime}$ on the other, one can see that all the commutation relations are the same except that the $\widehat{\nabla}^{i}$ s do not commute while the $\partial^{i}$ s do. The dynamical variables of the theory are the $c$-number coefficients appearing in the expansion (2.32) of the adjoint sections in terms of $Z_{i}$ 's. Since in the action, the constant curvature covariant derivatives only appear in commutators with the $Z_{i}$ 's and not with each other, substituting $U_{i}^{\prime}$ and $\partial^{\prime i}$ for $Z_{i}$ and $\widehat{\nabla}^{i}$ leaves the dynamics invariant. A similar construction was also considered in [38]. The integral and trace of the $U(n)$ theory can be translated to a $U(q)$ integral using the definition of the integral (2.61)

$$
\int d^{3} \widehat{\sigma} \frac{1}{\tilde{n}} \operatorname{tr} \Psi\left(Z_{i}\right)=\int d^{3} \sigma^{\prime} \operatorname{tr}_{q} \Psi\left(U_{i}^{\prime}\right)=(2 \pi)^{3} \sum_{a=1}^{q} \Psi_{000}^{a a}
$$

Making these substitutions one obtains the $U(q)$ action

$$
\mathcal{S}^{U(q)}=\frac{1}{g_{S Y M}^{\prime 2}} \int d t \int d^{3} \sigma^{\prime} \sqrt{\operatorname{det}\left(G^{\prime i j}\right)} \operatorname{tr}_{q}\left(\frac{1}{2} G_{i j}^{\prime} \mathcal{F}^{\prime 0 i} \mathcal{F}^{\prime 0 j}-\right.
$$




$$
\begin{gathered}
\frac{1}{4} G_{i j}^{\prime} G_{k l}^{\prime} \mathcal{F}^{i k} \mathcal{F}^{\prime j l}+ \\
\frac{1}{2} \sum_{a} \dot{X}^{a} \dot{X}^{a}-\frac{1}{2} \sum_{a} G_{i j}^{\prime}\left[D^{i}, X^{a}\right]\left[D^{\prime j}, X^{a}\right]+ \\
\left.\frac{1}{4} \sum_{a, b}\left[X^{a}, X^{b}\right]\left[X^{a}, X^{b}\right]+\text { fermions }\right),
\end{gathered}
$$

where

$$
D^{\prime i} \stackrel{\text { def }}{=} \partial^{\prime i}-i A^{\prime i}, \mathcal{F}^{\prime i j}=i\left[D^{\prime i}, D^{\prime j}\right]
$$

are the $U(q)$ connection and curvature. This shows that the original $U(n)$ theory is equivalent to a $U(q)$ NCSYM theory with gauge coupling given by (3.1) and defined on a trivial adjoint bundle over a noncommutative torus with deformation parameter $\Theta^{\prime}$ and metric given by (3.2).

In general two NCSYM theories are dual to each other if there exists an element $\Lambda$ of $S O(3,3 \mid \mathbf{Z})$ with Weyl spinor representation matrix $S$, such that their defining parameters are related as follows

$$
\begin{gathered}
\bar{\Theta}=(\mathcal{A} \Theta+\mathcal{B})(\mathcal{C} \Theta+\mathcal{D})^{-1}, \\
\left(\begin{array}{c}
\bar{n} \\
\bar{M}^{23} \\
\bar{M}^{31} \\
\bar{M}^{12}
\end{array}\right)=S\left(\begin{array}{c}
n \\
M^{23} \\
M^{31} \\
M^{12}
\end{array}\right), \\
\bar{G}^{i j}=(\mathcal{C} \Theta+\mathcal{D})_{k}^{i}(\mathcal{C} \Theta+\mathcal{D})^{j}{ }_{l} G^{k l}, \\
\bar{g}_{S Y M}^{2}=\sqrt{|\operatorname{det}(\mathcal{C} \Theta+\mathcal{D})|} g_{S Y M}^{2},
\end{gathered}
$$

where we used (2.49) in the last two equations. While $\Theta$ in (3.3) and the rank and magnetic flux numbers in (3.4) transform separately and the duality group action 
can be seen explicitly, the transformation of the gauge coupling and the metric also depends on $\Theta$. Note that $\mathcal{C} \Theta+\mathcal{D}$ satisfies a group property. If $\Lambda_{3}=\Lambda_{2} \Lambda_{1}$ and $\Theta^{\prime}=\Lambda_{1}(\Theta)$ then

$$
\mathcal{C}_{3} \Theta+\mathcal{D}_{3}=\left(\mathcal{C}_{2} \Theta^{\prime}+\mathcal{D}_{2}\right)\left(\mathcal{C}_{1} \Theta+\mathcal{D}_{1}\right)
$$

For a nonvanishing $\bar{n}$, the sign ambiguity that exists when one tries to associate to a $S O(3,3 \mid \mathbf{Z})$ transformation its spinor representation matrix, can be removed by requiring that $\bar{n}$ is positive. Strictly speaking, one should not consider duality transformations for which $\bar{n}$ vanishes since in this case the description in terms of gauge theories becomes singular. 


\subsection{Target Space Duality}

Next we show that the $S O(3,3 \mid \mathbf{Z})$ duality discussed in the previous section is the realization in NCSYM gauge theories of T-duality in the auxiliary Type IIA string theory. This relation is described by the following diagram.

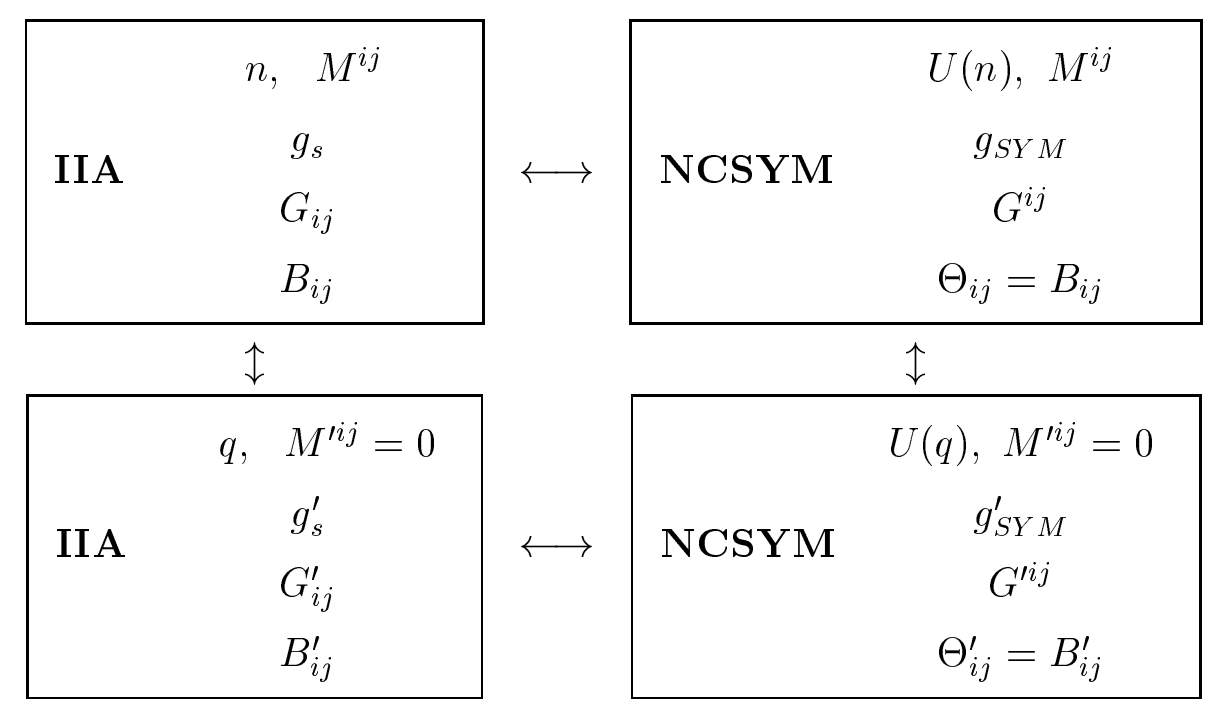

The right side of the diagram shows the equivalence described in Section (3.1). The horizontal arrows represent the Connes, Douglas and Schwarz conjecture [27]. The left side of the diagram contains the string coupling, D brane charges, and compactification moduli of the two auxiliary Type IIA string theories corresponding to the NCSYM's on the right. The additional moduli corresponding to Ramond-Ramond backgrounds are set to zero in this chapter and will be considered separately in Chapter 4. Note that the NCSYM metric is the inverse of 
the Type IIA metric as indicated by the index position, the deformation parameter equals the NS antisymmetric tensor, and the rank and magnetic flux numbers translate into D0 brane number and D2 brane winding. Finally the SYM and string coupling are related by (2.5).

For compactification on $T^{2}$, the duality transformation (2.54) can be written as an $S L(2, \mathbf{Z})$ mapping class group transformation conjugated by a T-duality in the $x^{1}$ direction ${ }^{1}$. The sequence of duality transformations is shown in figure 3.1. Under T-duality in the $x^{1}$-direction the two D0-branes are mapped into D1-strings wrapping the horizontal cycle and the D2-brane into a D1 string wrapping the oblique cycle. The T-dual torus is not rectangular for a nonvanishing $\theta$. In fact the D1-strings can be in a lower energy state obtained by minimizing their total length. This corresponds to the fact that the original D0 and D2-branes form a bound state. Under the $S L(2, \mathbf{Z})$ mapping class group transformation we can arrange the D1-string along the horizontal axis of the torus. This is just a relabeling of the defining 1-cycle of the torus. Finally after another T-duality in the $x^{1}$-direction we arrive at the final configuration which contains a single D0-branes and the final NS-NS modulus given by $\theta^{\prime}$.

In the remainder of this section we calculate the relation between the parameters of the two auxiliary Type IIA string theories. First we describe how the metric, antisymmetric tensor and the string coupling transform under an arbitrary

\footnotetext{
${ }^{1}$ The T-duality in the $x^{1}$-direction is an element of $O(2,2 \mid \mathbf{Z})$ of negative determinant.
} 

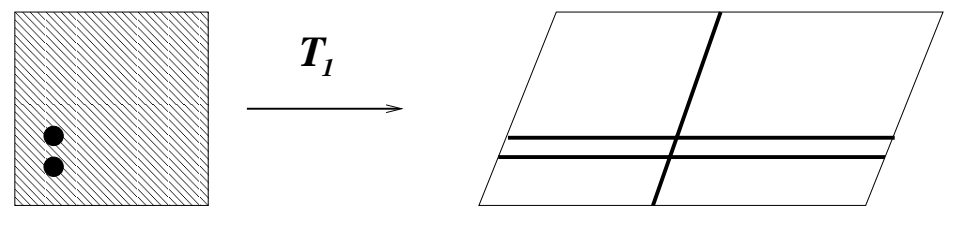

$$
n=2 \quad m=1
$$

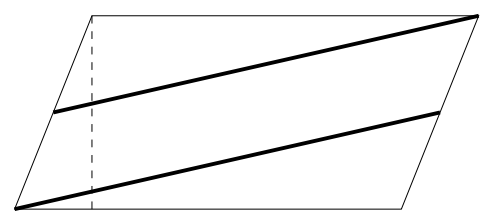

$\Theta$ $S L(2, Z)$

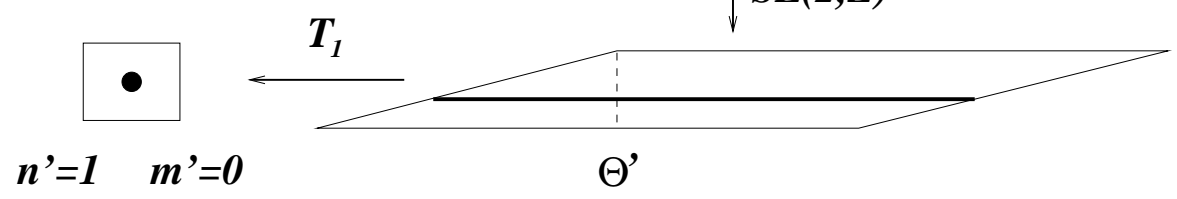

Figure 3.1: The covering space of $T^{2}$ with one D0-brane per cell

T-duality transformation, and then take the limit

$$
\alpha^{\prime} \rightarrow 0, \quad G_{i j} \rightarrow 0
$$

keeping $\alpha^{\prime-2} G_{i j}$ constant. This is the limit proposed by Seiberg and Sen [18, 17] and briefly discussed in the introduction. However, in this limit the auxiliary Type IIA string metric vanishes. Instead we calculate directly the inverse metric of the NCSYM theory which, after including factors of $\alpha^{\prime}$, is given by $\alpha^{\prime-2} G_{i j}$.

Under the T-duality group $S O(d, d \mid \mathbf{Z})$, the metric and NS antisymmetric tensor $^{2}$ transform together by fractional transformations [71]

$$
G^{\prime}+B^{\prime}=(\mathcal{A}(G+B)+\mathcal{B})(\mathcal{C}(G+B)+\mathcal{D})^{-1}
$$

Using the identification between $\Theta$ and $B$ one obtains $H^{-1}=\mathcal{C} B+\mathcal{D}$. Then,

\footnotetext{
${ }^{2}$ Hopefully, there is no confusion between $B$, denoting the NS tensor, and $\mathcal{B}$, which is the
} upper right block of $\Lambda$. 
after some matrix algebra, one can write the symmetric and antisymmetric part of (3.9) as

$$
\begin{aligned}
G^{\prime} & =H^{T} G\left(1-(H \mathcal{C} G)^{2}\right)^{-1} H \\
B^{\prime} & =(\mathcal{A} B+\mathcal{B})(\mathcal{C} B+\mathcal{D})^{-1}-H^{T} G H \mathcal{C} G\left(1-(H \mathcal{C} G)^{2}\right)^{-1} H
\end{aligned}
$$

One derives this using the fact that $H \mathcal{C}$ is antisymmetric. This can be shown using

$$
(\mathcal{C} B+\mathcal{D})^{-1}=\left(\mathcal{A}-(\mathcal{A} B+\mathcal{B})(\mathcal{C} B+\mathcal{D})^{-1} \mathcal{C}\right)^{T}
$$

which follows from (2.44). Note that (3.10) and (3.11) have simple expansions in $G$. For an elementary T-duality in the $x^{1}$ direction the string coupling constant transforms as

$$
g_{S}^{\prime}=g_{S} G_{11}^{-1 / 2}
$$

Taking the limit (3.8) in (3.11) one can see that the antisymmetric tensor itself transforms by fractional transformation ${ }^{3}$

$$
B^{\prime}=(\mathcal{A} B+\mathcal{B})(\mathcal{C} B+\mathcal{D})^{-1}
$$

To find the duality transformation of the metric, we reinstate factors of $\alpha^{\prime}$ in (3.10) since the $S O(3,3 \mid \mathbf{Z})$ transformations are defined to act on dimensionless fields. Now, take the limit (3.8) and to first order in the dimensionless metric $\alpha^{\prime-1} G_{i j}$ one has

$$
\alpha^{\prime-2} G^{\prime}=(\mathcal{C} B+\mathcal{D})^{-T}\left(\alpha^{\prime-2} G\right)(\mathcal{C} B+\mathcal{D})^{-1} .
$$

\footnotetext{
${ }^{3}$ This is consistent with the fact that the action by fractional transformations preserves the antisymmetry of the matrices.
} 
Making the identification $B=\Theta$, one recognizes above the $H$ matrix defined in $(2.49)$.

Finally, using (3.12), one can also calculate how the string coupling transforms under duality. It was shown in [34] that the $S O(d, d \mid \mathbf{Z})$ group is generated by a set of simple elements. These are written explicitly in the Appendix A. For each of these generators, one can check using (3.12) that the string coupling transforms as

$$
g_{S}^{\prime}=g_{S}|\operatorname{det}(\mathcal{C} B+\mathcal{D})|^{-1 / 2} \text {. }
$$

In fact (3.15) is true for an arbitrary transformation because $\mathcal{C} B+\mathcal{D}$ satisfies the group property $(3.7)$.

Comparing the T-duality relations (3.13), (3.14) and (3.15) with the NCSYM duality relations (3.3), (3.5) and (3.6), using (2.5) to relate the string and gauge couplings, one sees that indeed the two dualities coincide.

\subsection{Projective Modules and Morita Equivalence}

A quantum vector bundle is a projective $\mathcal{A}$-module $\mathcal{E}$. First, consider the classical commutative picture. The set $\mathcal{E}$ of global sections of a vector bundle over a base space $X$ has the structure of a projective module over the algebra $C(X)$. Having a module essentially means that one can add sections and can multiply them by functions. Not all modules over a commutative algebra are vector bundles. For example, the set of sections on a space consisting of a collection of fibers of 
different dimensions over a base space also form a module. However, projective modules over the algebra of functions on a topological space are in one to one correspondence with vector bundles over that space. By definition, a projective module is a direct summand in a free module. A free module $\mathcal{E}_{0}$ over an algebra $\mathcal{A}$ is a module isomorphic to a direct sum of a finite number of copies of the algebra

$$
\mathcal{E}_{0}=\mathcal{A} \oplus \ldots \oplus \mathcal{A} .
$$

Trivial bundles correspond to free modules since the description of their sections in terms of components is global, and each component is an element of $C(X)$. For every vector bundle, one can find another one such that their direct sum is a trivial bundle. In dual language this implies that the module of sections $\mathcal{E}$ is projective

$$
\mathcal{E}_{0}=\mathcal{E} \oplus \mathcal{E}^{\prime}
$$

Again it is nontrivial to show the converse, that every projective module is isomorphic to the set of sections of some vector bundle. Finally, projective modules over noncommutative algebras are the quantum version of vector bundles.

In the noncommutative case, one distinguishes between left and right projective modules. Multiplying fundamental sections from the right with elements of $\mathcal{A}_{\Theta}$ preserves the boundary conditions (2.15), while multiplication on the left gives something that is no longer a global section. Thus the set of sections of the fundamental bundle form a right projective module over the $\mathcal{A}_{\Theta}$ algebra which I will denote $\mathcal{F}_{\eta}^{\ominus}$. This is no longer true for the adjoint sections, since in (2.16) the 
transition functions multiply from both the left and right. However, one can check that the adjoint sections are both left and right projective modules over the $\mathcal{A}_{(-\Theta)}$ algebra. Also a fundamental section is a left projective modules over the $\mathcal{A}_{(-\Theta)}$ algebra. This is because the exponents of the $\mathcal{U}_{i}$ 's satisfy

$$
\left[i \sigma_{i}-2 \pi \Theta_{i j} \partial^{j}, \sigma_{k}\right]=0
$$

thus the $\mathcal{U}_{i}$ 's can be commuted over the transition functions in (2.15) and (2.16). Additionally, the fact that $\mathcal{F}_{n, m}^{\Theta}$ is both a left $\mathcal{A}_{(-\Theta)}$-module and a right $\mathcal{A}_{\Theta}$-module can be understood as follows. Since $\left[\mathcal{U}_{i}, \sigma_{j}\right]=0$ one also has

$$
\mathcal{U}_{i} \Phi\left(\sigma_{i}\right)=\Phi\left(\sigma_{i}\right) U_{i}
$$

where we dropped the derivatives when there was nothing to their right. Let $a$ be an element of $\mathcal{A}_{(-\Theta)}$

$$
a=\sum_{i_{1} \ldots i_{d} \in \mathbf{Z}} a_{i_{1} \ldots i_{d}} \mathcal{U}_{1}^{i_{1}} \ldots \mathcal{U}_{d}^{i_{d}}
$$

Thus multiplying on the left with $a$ is equivalent to multiplying on the right with $\widetilde{a}$

$$
a \Phi=\Phi \widetilde{a},
$$

where $\widetilde{a}=\sum_{i_{1} \ldots i_{d} \in \mathbf{Z}} a_{i_{1} \ldots i_{d}} U_{d}^{i_{d}} \ldots U_{1}^{i_{1}}$ is the same function as a but with $\mathcal{U}_{i}$ 's as arguments and with all the factors written in reversed order.

In the remainder of this chapter, we only consider the two dimensional case and use the gauge (2.14). The construction in Chapter 2 is equivalent to the projective modules discussed in [27]. By solving the boundary conditions one goes 
from a local to a global description. Here we present explicit formulae for this equivalence. First one has to express the left actions on the fundamental sections as actions on the Hilbert space [36]. For example the action of the $Z_{i}$ generators is given by

$$
\left(Z_{1} \widehat{\phi}\right)_{j}(x)=\widehat{\phi}_{j-a}\left(x-\frac{1}{m}\right), \quad\left(Z_{2} \widehat{\phi}\right)_{j}(x)=e^{-2 \pi i j / m} e^{2 \pi i x /(n+m \theta)} \widehat{\phi}_{j}(x) .
$$

This can be written as

$$
Z_{1}=W_{1}^{a} V_{1}, \quad Z_{2}=W_{2} V_{2}
$$

where $V_{i}$ and $W_{i}$ are operators acting on the Hilbert space as

$$
\begin{gathered}
\left(V_{1} \widehat{\phi}\right)_{j}(x)=\widehat{\phi}_{j}\left(x-\frac{1}{m}\right), \quad\left(V_{2} \widehat{\phi}\right)_{j}(x)=e^{2 \pi i x /(n+m \theta)} \widehat{\phi}_{j}(x), \\
\left(W_{1} \widehat{\phi}\right)_{j}(x)=\widehat{\phi}_{j-1}(x), \quad\left(W_{2} \widehat{\phi}\right)_{j}(x)=e^{-2 \pi i j / m} \widehat{\phi}_{j}(x) .
\end{gathered}
$$

These operators satisfy the following relations

$$
V_{1} V_{2}=e^{-2 \pi i /[m(n+m \theta)]} V_{2} V_{1}, \quad W_{1} W_{2}=e^{2 \pi i / m} W_{2} W_{1}, \quad\left[V_{i}, W_{j}\right]=0
$$

and can be used to express other operators acting in the Hilbert space. For example $\mathcal{U}_{1}=W_{1} V_{1}^{n+m \theta}$ and $\mathcal{U}_{2}=W_{2}^{n} V_{2}^{n+m \theta}$.

Now we present the correspondence between [27] and the approach followed here ${ }^{4}$. The two integers $p$ and $q$ and the angular variable $\theta_{\text {CDS }}$ labeling the projective module $\mathcal{H}_{p, q}^{\theta_{\mathrm{CDS}}}$ of [27], and $\theta_{\mathrm{CDS}}^{\prime}$ can be expressed in terms of the quantities used here

$$
p=n, \quad q=-m, \quad \theta_{\mathrm{CDS}}=\Theta_{12}, \quad \theta_{\mathrm{CDS}}^{\prime}=\Theta_{12}^{\prime} .
$$

\footnotetext{
${ }^{4}$ We follow here the same notation as in $[36,40]$ except for an overall minus sign in the definition of $\theta$.
} 
Then $\mathcal{F}_{n, m}^{\Theta} \cong \mathcal{H}_{n,-m}^{\Theta_{12}}$. The Hilbert space representation of [27] written in terms of the function $f(s, k)$ with $s \in \mathbf{R}$ and $k \in \mathbf{Z}_{q}$ is linearly related to the $\widehat{\phi}_{k}(x)$ representation

$$
\widehat{\phi}_{k}(x)=\sum_{l=1}^{m} \mathcal{K}_{k l}\left(\mathcal{S}_{\left(-\frac{m}{n+m \theta}\right)} f\right)(x, l) .
$$

Here $\mathcal{K}$ is an $m \times m$ representation changing matrix defined as in (2.20) but for $m$-dimensional 't Hooft matrices, and $\mathcal{S}_{\lambda}$ is the rescaling operator $\left(\mathcal{S}_{\lambda} f\right)(x, k)=$ $f(\lambda x, k)$ which can be expressed using the ordered exponential

$$
\mathcal{S}_{\lambda}=\lambda E\left((\lambda-1) x, \partial_{x}\right)
$$

Also, using lower case to distinguish them from our current notation which follows [36], the operators in [27] represented in the $\hat{\phi}_{k}(x)$ basis are given by

$$
\begin{gathered}
v_{0}=V_{2}^{n+m \theta}, \quad v_{1}=V_{1}^{n+m \theta}, \quad w_{0}=e^{2 \pi i n / m} W_{2}^{n}, \quad w_{1}=e^{2 \pi i / m} W_{1} \\
z_{0}=e^{2 \pi i / m} Z_{2}, \quad z_{1}=e^{-2 \pi i a / m} Z_{1}^{-1}, \quad u_{0}=e^{2 \pi i n / m} \mathcal{U}_{2}, \quad u_{1}=e^{2 \pi i / m} \mathcal{U}_{1} .
\end{gathered}
$$

Next we introduce the Morita equivalence of two algebras [42, 43, 34, 38], which can be used to describe a subgroup of the T-duality group of the M-theory compactification in the language of noncommutative SYM gauge theory.

Two $C^{*}$-algebras $\mathcal{A}$ and $\mathcal{A}^{\prime}$ are Morita equivalent if there exists a right $\mathcal{A}$ module $\mathcal{E}$ such that the algebra $E n d_{\mathcal{A}} \mathcal{E}$ is isomorphic to $\mathcal{A}^{\prime}$. Here $E n d_{\mathcal{A}} \mathcal{E}$ denotes the set of endomorphisms of the $\mathcal{A}$-module $\mathcal{E}$. It consists of linear maps $T$ on $\mathcal{E}$ where linearity is not only with respect to $c$-numbers but also with respect to right multiplication by elements of $\mathcal{A}$

$$
T(\Phi f)=T(\Phi) f, \quad \Phi \in \mathcal{E}, \quad f \in \mathcal{A} .
$$


An example of Morita equivalent algebras is $\mathcal{A}_{\Theta}$ and $\mathcal{A}_{\Theta^{\prime}}$. As discussed above, the projective module associated to the quantum fundamental bundle $\mathcal{F}_{n, m}^{\Theta}$ is a right $\mathcal{A}_{\Theta}$-module. One can prove that $E n d_{\mathcal{A}_{\Theta}} \mathcal{F}_{n, m}^{\Theta}$ is isomorphic to $\mathcal{A}_{\Theta^{\prime}}$. Here we just show that the two algebras have the same generators. Using (3.17) one has $T(\Phi \widetilde{a})=T(a \Phi)$ and $T(\Phi) \widetilde{a}=a T(\phi)$ and since $T$ is an endomorphism one obtains $T(a \Phi)=a T(\phi)$, which can also be written as $[T, a]=0$. But the $Z_{i}$ 's were found exactly by requiring that they commute with $\mathcal{U}_{i}^{\prime}$ 's so $T \in \mathcal{A}_{\Theta^{\prime}}$.

The physical interpretation of Morita equivalence is that a $U(n)$ SYM gauge theory on the twisted bundle with magnetic flux $m$ is equivalent to a $U(1)$ gauge theory on a dual quantum torus $\mathcal{A}_{\Theta^{\prime}}$. This can be seen as a consequence of the discussion following equation (2.13). The gauge field components $A^{i}$, the scalar fields $X^{a}$, and the components of $\Theta$ are not matrix valued, rather they are onedimensional. The final result is a matrix because the $Z_{i}$ 's are matrices. On the other hand, one can ignore the internal structure of the $Z_{i}$ 's and just regard them as the generators of $\mathcal{A}_{\Theta^{\prime}}$, thus allowing us to reinterpret the original theory as a noncommutative $U(1)$ gauge theory on the quantum torus $\mathcal{A}_{\Theta^{\prime}}$.

Since $\Theta$ is a continuous variable, one can interpolate continuously, through noncommutative SYM theories, between two commutative SYM theories with gauge groups of different rank and appropriate magnetic fluxes. This $S L(2, \mathbf{Z})$ duality subgroup has a nice geometric interpretation in the T-dual picture of [28] where it corresponds to a change of basis of the dual torus lattice [32]. 


\subsection{Gauge Transformations}

In this section, we consider a gauge equivalent formulation of the previous results closely following the treatment of Taylor in [70] of the corresponding commutative case. In that paper a gauge transformation was considered so as to change the standard 't Hooft transition function into trivial transition function in the $X^{2}$ direction. When the transition functions are trivial T-duality has the standard form, i.e. the gauge field translates directly into the position of a Dstring on the dual torus. A similar gauge transformation can be performed in the noncommutative case.

First let us consider a general gauge transformation $g\left(\sigma_{1}, \sigma_{2}\right)$. Just as in the classical case the covariant derivatives transform as $D_{i}^{\prime}=g^{-1} D_{i} g$ resulting in the following transformation for the gauge fields

$$
A^{i i}=g^{-1} A^{i} g+i g^{-1} \partial^{i} g .
$$

As a result the new transition functions are given by

$$
\begin{aligned}
& \Omega_{1}^{\prime}\left(\sigma_{1}, \sigma_{2}\right)=g^{-1}\left(\sigma_{1}+2 \pi, \sigma_{2}\right) \Omega_{1}\left(\sigma_{1}, \sigma_{2}\right) g\left(\sigma_{1}, \sigma_{2}\right), \\
& \Omega_{2}^{\prime}\left(\sigma_{1}, \sigma_{2}\right)=g^{-1}\left(\sigma_{1}, \sigma_{2}+2 \pi\right) \Omega_{2}\left(\sigma_{1}, \sigma_{2}\right) g\left(\sigma_{1}, \sigma_{2}\right) .
\end{aligned}
$$

Again all this is just as in the classical case except that one has to take into account the noncommutativity of the $\sigma_{i}$ 's.

It will be useful to consider first the $\Theta=0$ commutative case. Then one knows 
both the original gauge fields (2.14) and the transformed ones

$$
A^{\prime 1}=0, \quad A^{\prime 2}=\frac{m}{n} \frac{\sigma_{1}}{2 \pi}+Q
$$

where $Q=\frac{1}{n} \operatorname{diag}(0,1, \ldots, n-1)$, and I use primes for all variables in the new gauge. Than the differential equation for the gauge transformation is

$$
\partial^{1} g=0, \quad i \partial^{2} g=g A^{\prime 2}-A^{2} g=g Q
$$

which can be integrated to give

$$
g=K e^{-i \sigma_{2} Q}
$$

where the integration constant $K$ is the $n \times n$ matrix (2.20). It was fixed by requiring a trivial $\Omega_{2}^{\prime}$ as given by (3.19). Using the gauge transformation (3.20) one can can now calculate both transition functions

$$
\Omega_{1}^{\prime}=e^{2 \pi i m / n} e^{i \sigma_{2} T_{m}} V^{m}, \quad \Omega_{2}^{\prime}=1
$$

where $T_{k}=\operatorname{diag}(0, \ldots, 0,1, \ldots, 1), k=1, \ldots, n$ with the first $n-k$ entries vanishing and the last $k$ equal to unity.

Next we discuss the noncommutative case. The first thing to notice is that the original quantum transition functions (2.18) are $\theta$ independent and only contain the $\sigma_{2}$ variable. Similarly the classical gauge transformation (3.20) only depends on $\sigma_{2}$ so that the classical computation of the new transition functions is also valid in the quantum case. Using (3.18) the new gauge fields are given by

$$
A^{\prime 1}=0, \quad A^{\prime 2}=\frac{m}{n+m \theta} \frac{\sigma_{1}}{2 \pi}+\frac{n}{n+m \theta} Q
$$


Since (3.16) implies $\left[\mathcal{U}_{i}, g\right]=0$ one can see that the gauge transformation is compatible with the quotient conditions (2.2). One can use the gauge transformation to obtain the generators of the sections of the adjoint bundle

$$
Z_{1}^{\prime}=e^{i \sigma_{1} /(n+m \theta)} e^{-2 \pi i n \theta^{\prime} Q}, \quad Z_{2}^{\prime}=e^{2 \pi i / n} V e^{i \sigma_{2}\left(1-T_{n-1}\right)} .
$$

The explicit formulae for the fundamental sections in the new gauge is

$$
\Phi_{k}^{\prime}\left(\sigma_{1}, \sigma_{2}\right)=\sum_{r \in \mathbf{Z}} e^{i \sigma_{2} r} \chi_{k-n r}\left(\frac{\sigma_{1}}{2 \pi}+\frac{k-n r}{m}\right) .
$$

The $\chi_{s}$ functions are defined over the real axis and must satisfy

$$
\chi_{s+m}(x)=e^{-2 \pi i m / n} \chi_{s}(x),
$$

so that only $m$ of them are independent. Again, note that since the transition functions only contain $\sigma_{2}$ and all were ordered to the left of $\sigma_{1}$ in the solution for the sections of the fundamental bundle, they have the same form in the noncommutative and in the classical case. 


\section{Chapter 4}

\section{Adding Ramond-Ramond Backgrounds}

For $d \geq 2$, in addition to the compactification metric there are moduli which, in terms of the auxiliary Type IIA string theory $[17,18]$, correspond to the 2form of the NS-NS (Neveu Schwarz-Neveu Schwarz) sector and the R-R (RamondRamond) forms. Next we will extend the result of Chapter 2 by allowing arbitrary R-R backgrounds.

In Section 4.1, we review the transformation properties of the R-R moduli under the duality group $[72,73,74]$. The dimensionally reduced action of Type IIA supergravity is invariant under the T-duality group ${ }^{1} S O(d, d)$. By deriving the nonlinear sigma model which describes the scalar fields of the supergravity, one can extract the transformation properties of the R-R backgrounds under the duality group. In particular, we will show that appropriately defined fields, which are combinations of the R-R forms and the NS-NS two-form, transform in a spinor representation of the duality group.

In Section 4.2, we will identify the Chern-Simon parameters of the gauge theory with the R-R moduli and then show that the duality transformations relating different NCSYM theories can be extended to include these terms. In the pro-

\footnotetext{
${ }^{1}$ The equations of motion are invariant under the full U-duality group $E_{d+1(d+1)}$.
} 
cess, we will also obtain the transformation properties of the parameters and show that they coincide with the transformations expected from string theory and derived in Section 4.1 using the dimensional reduction of 10-dimensional Type IIA supergravity.

Finally, in Appendix B we present some results, used in the main text, regarding transformation properties under the T-duality group in the limit of small compactification volume and decoupling of string excitations.

A similar proposal for the additional terms in the noncommutative action was made in [50].

\subsection{Duality of Seven Dimensional Supergravity}

Type IIA superstring theory compactified on a d-dimensional torus is invariant under the T-duality group $S O(d, d \mid \mathbf{Z})$. The low energy supergravity effective action describing this compactification is in fact invariant under the continuous group $S O(d, d)$. This action can be obtained directly from the 10-dimensional Type IIA supergravity by dimensional reduction. In this section we derive the transformation properties of the R-R moduli under the discrete duality group. Since this is a subgroup of the corresponding continuous group which is a symmetry of the low energy 10-dimensional supergravity action, one can obtain these transformation properties by analyzing the symmetries of the the nonlinear sigma model which describes the dynamics of the scalars in the supergravity action.

The NS-NS scalars are described locally by an $O(d, d) / O(d) \times O(d)$ nonlinear 
sigma model. Taking into account the T-duality group, the NS-NS nonlinear sigma model is in fact defined on

$$
O(d, d \mid \mathbf{Z}) \backslash O(d, d) / O(d) \times O(d) .
$$

On the other hand simple counting arguments suggest that the R-R scalar fields transform in a chiral spinor representation of the duality group. This statement is almost correct except that the fields which transform in the spinor representation are some redefined fields involving not only the R-R fields but also the NS-NS two form.

The 10-dimensional supergravity action written in terms of the string metric is given by

$$
\begin{gathered}
\mathcal{S}=\int d^{10} x \sqrt{g} e^{-2 \phi}\left(R+4(\nabla \phi)^{2}-\frac{1}{2 \cdot 3 !} H^{2}\right) \\
-\int d^{10} x \sqrt{g}\left(\frac{1}{2 \cdot 2 !} F^{2}+\frac{1}{2 \cdot 4 !} F^{2}\right) \\
-\frac{1}{4} \int F_{(4)} \wedge F_{(4)} \wedge B+\ldots,
\end{gathered}
$$

where we have not written the terms containing the fermionic fields. The first line contains only NS-NS fields while the second contains the kinetic terms of the R-R forms. The various field strengths are defined as follows

$$
\begin{aligned}
H & =d B, \\
F & =d A_{(1)}, \\
F_{(4)} & =d A_{(3)}, \\
F^{\prime} & =F_{(4)}+A_{(1)} \wedge d B,
\end{aligned}
$$


where the subscript indicates the rank of the form. Note that R-R fields couple to the NS-NS fields through the metric and through the $F^{\prime 2}$ term, which depends on the antisymmetric NS-NS two-form.

Next we perform the dimensional reduction along coordinates $x^{i}$ for $i=1,2,3$. The massless scalars from the NS sector can be organized in the symmetric ma$\operatorname{trix}[75]$

$$
\mathcal{M}=\left(\begin{array}{cc}
G^{-1} & -G^{-1} B \\
B G^{-1} & G-B G^{-1} B
\end{array}\right) .
$$

Note that $\mathcal{M}$ is also an element of the group $S O(3,3)$. Using a result from Appendix $\mathrm{B}$, one can obtain the Weyl spinor representation of $\mathcal{M}$

$$
S(\mathcal{M})=\left(\begin{array}{cc}
\operatorname{det} G^{-1 / 2} & \operatorname{det} G^{-1 / 2} b^{T} \\
\operatorname{det} G^{-1 / 2} b & \operatorname{det} G^{1 / 2} G^{-1}+\operatorname{det} G^{-1 / 2} b b^{T}
\end{array}\right)
$$

where $b=* B$, and the star denotes the operator which transforms an antisymmetric matrix into its dual column matrix. The star operator always dualizes only with respect to the compactified coordinates.

One obtains additional scalars from the dimensional reduction of R-R forms. As mentioned above these fields do not have simple transformation properties under the T-duality group but one can define the following odd rank forms

$$
\begin{aligned}
& C_{(1)}=A_{(1)}, \\
& C_{(3)}=A_{(3)}-A_{(1)} \wedge B,
\end{aligned}
$$

and organize them in a column matrix which, as will be seen shortly, transforms 
in a chiral spinor representation of the duality group

$$
\chi=\left(\begin{array}{c}
C_{123} \\
C_{1} \\
C_{2} \\
C_{3}
\end{array}\right) .
$$

The other fields can also be organized in representations of the duality group such that the action obtained by dimensional reduction from 10-dimensional supergravity is explicitly invariant. The six vectors obtained from the dimensional reduction of NS-NS fields transform in the fundamental representation while the 7-dimensional dilaton and the 7-dimensional space-time metric and 2-form are singlets. The four vectors obtained from the R-R forms transform in a chiral spinor representation and, after dualizing the 3-form, the rest of the bosonic fields form a chiral spinor of 2-forms.

For our purpose, it will be enough to consider the nonlinear sigma model part of the action containing the kinetic terms of the scalar fields of the theory

$$
\mathcal{S}=\frac{1}{2} \int d^{7} x \sqrt{\widetilde{g}}\left(e^{-2 \Phi} \widetilde{g}^{\mu \nu} \operatorname{tr}\left(\partial_{\mu} \mathcal{M}^{-1} \partial_{\nu} \mathcal{M}\right)+\widetilde{g}^{\mu \nu} \partial_{\mu} \chi^{T} S(\mathcal{M}) \partial_{\nu} \chi\right)+\ldots
$$

where $\widetilde{g}_{\mu \nu}$ and $\Phi$ are the 7-dimensional metric and dilaton, and we have not written the kinetic term for the dilaton. The nonlinear sigma model part of the action is written in a form that is explicitly invariant under $S O(3,3)$ and in fact the whole supergravity action could be written in invariant form. The duality transformations of the scalar fields are given by

$$
\begin{aligned}
\overline{\mathcal{M}} & =\Lambda^{-T} \mathcal{M} \Lambda^{-1}, \\
\bar{\chi} & =S(\Lambda) \chi .
\end{aligned}
$$


To prove the invariance of the action we used $S\left(\Lambda^{T}\right)=S(\Lambda)^{T}$.

The main purpose of this section was to obtain the relations (4.2) which show how the fields $\chi$ with simple transformations properties under the T-duality group are related to the R-R forms.

\subsection{T-duality of the Chern-Simon Type Terms}

In this section, we discuss how to modify the NCSYM action so that it describes the DLCQ of M-theory in the presence of arbitrary moduli. In the auxiliary Type IIA string theory the additional moduli are constant R-R backgrounds corresponding to generalized Wilson lines. Then we show that the action which includes the new terms is also invariant under the duality group $S O(3,3 \mid \mathbf{Z})$ and that the parameters of the new terms transform exactly as expected from string theory.

First we will guess the form of these terms using our experience with the commutative case which corresponds to a vanishing NS-NS background 2-form $B$. In this case the compactified Matrix model corresponding to $n$ D0-branes is described by a $U(n)$ supersymmetric Yang-Mills theory. This is obtained by performing a T-duality transformation along all the compact directions. However,

for nonvanishing R-R moduli, the action contains an additional Chern-Simon type term $[76,77,78,79,80]$

$$
\mathcal{S}_{C S}=\frac{1}{4(2 \pi)^{3}} \int \operatorname{tr}\left(e^{2 \pi \mathcal{F}} \sum_{k \text { odd }} A^{(k)}\right),
$$


where $A^{(k)}$ are the the T-dual R-R fields. Note also that under T-duality in all directions the dual of $B$ also vanishes if $B$ was zero. This is why only $\mathcal{F}$ appears in the exponent while in general one would also subtract the dual of $B$.

Next, consider the effect of a nonvanishing $B$ on this action. If $\gamma^{i j}$ represents a two cycle wrapped around directions $x^{i}$ and $x^{j}$, then the deformation parameters are defined by

$$
\Theta_{i j}=\frac{1}{(2 \pi)^{2}} \int_{\gamma^{i j}} B
$$

In the super Yang-Mills part of the action the only change required by a nonvanishing $B$ was to make the coordinates noncommutative with deformation parameter $\Theta$. The metric and gauge coupling constant are the same as those obtained by T-duality from the Matrix model for a vanishing NS-NS 2-form. We emphasize that the metric of the NCSYM gauge theory is not the T-dual metric obtained by first taking the inverse of $E=G+\Theta$ and then extracting the symmetric part. The NCSYM metric $G^{i j}$ is just the inverse of the original metric. Thus one must distinguish between a T-duality in all directions and the noncommutative Fourier transformation relating the Matrix model and the NCSYM gauge theory.

Let us explain why the NCSYM metric is $\Theta$ independent. To compactify the Matrix model on a torus we will first consider the Matrix model on the covering space and then impose a quotient condition. If the $B$ modulus is nonvanishing, after going to the topologically trivial covering space, it can be gauged away. However this gauge transformation does not leave the wave functions of strings invariant and thus one must transform the translation operators implementing 
the quotient condition. The new translation operators do not commute and their noncommutativity is measured by $\Theta$.

Imposing the new quotient conditions on the Matrix action results directly in the NCSYM gauge theory. The only difference with the $B=0$ case is that one has to use noncommutative Fourier transformations instead of the standard Fourier transformations when going from the Matrix model to the NCSYM gauge theory. This however does not result in a different metric and gauge coupling constant. The main point of this discussion was to show that one can trade a nonvanishing $B$ field for noncommutative coordinates on the dual super Yang-Mills gauge theory.

We assume that the parameters of the Chern-Simon terms are also the same as for vanishing $\Theta$, except that the new terms are defined on a noncommutative torus. In particular for compactification on a three torus one has

$$
\mathcal{S}_{C S}=\frac{1}{4(2 \pi)^{3}} \int \operatorname{tr}\left(2 \pi \mathcal{F} \wedge A^{(2)}+\frac{1}{2} 2 \pi \mathcal{F} \wedge 2 \pi \mathcal{F} \wedge A^{(0)}\right)
$$

Just as in the commutative case these terms are topological, supersymmetric and gauge invariant. In this action $\Theta$ only appears through the noncommutativity of the coordinates and $A^{(0)}$ and $A^{(2)}$ are the T-dual R-R forms ${ }^{2}$ calculated as if the NS-NS 2-form vanishes

$$
A^{(0)}=* A_{(3)}, \quad A^{(2)}=-* A_{(1)}
$$

The 1-form R-R field $A_{(1)}$ has a lower index and should not be confused with

\footnotetext{
${ }^{2}$ When we write the R-R forms in components we will drop the rank of the form as it is possible to identify the form from the position and number of indices.
} 
the Yang-Mills gauge field $A^{i}$. With this distinction in mind we will write the action (4.4) in the dual Matrix theory language using the R-R backgrounds on the original torus

$$
\mathcal{S}_{C S}=\int d t \operatorname{Tr}\left(\dot{X}^{i} A_{i}+\frac{i}{2 \pi} \dot{X}^{i} X^{j} X^{k} A_{i j k}\right)
$$

where $\operatorname{Tr}$ is the formal trace over infinite dimensional matrices divided by the infinite order of the quotient group [25]. It is convenient to write the action in component notation

$$
\mathcal{S}_{C S}=\frac{1}{2} \int d t \int \frac{d^{3} \sigma}{(2 \pi)^{3}} \operatorname{tr}\left(\varepsilon_{i j k}\left(2 \pi \mathcal{F}^{0 i} A^{j k}+(2 \pi)^{2} \mathcal{F}^{0 i} \mathcal{F}^{j k} A\right)\right)
$$

where the magnetic and electric field strengths in the temporal gauge are

$$
\mathcal{F}^{0 i}=i\left[\partial^{0}, D^{i}\right], \quad \mathcal{F}^{i j}=i\left[D^{i}, D^{j}\right]
$$

In the original conjecture [15], the large $N$ limit of Matrix theory describes the infinite momentum frame of M-theory. Large $N$ corresponds to a decompactification of the light-cone direction and in this limit the $\Theta$ and $A_{(1)}$ can be set to zero. Note that in M-theory these moduli correspond to $C_{-i j}$ and $g_{-i}$ and can be eliminated by a gauge transformation and a reparametrization when $x^{-}$is noncompact. In that case only the last term in (4.5) survives, the action becomes commutative and reduces to the action considered in [80, 81].

The action (4.5) is invariant under the $S O(3,3 \mid \mathbf{Z})$ duality group of the auxiliary string theory. Consider a Chern-Simon type action defined on a $\eta$-bundle. Here $\eta$ is a $S O(3,3 \mid \mathbf{Z})$ spinor containing the rank of the group and the magnetic flux numbers. We perform the same sequence of field redefinitions used in [47], where it 
was shown explicitly for the case of vanishing R-R moduli, that the $U(n)$ NCSYM action is equivalent to a $U(q)$ NCSYM action on a trivial bundle, where $q$ is the greatest common divisor of $n$ and the magnetic fluxes $M$. Let $H=(\mathcal{C} \Theta+\mathcal{D})^{-1}$ be the matrix defined in [47], where $\mathcal{C}$ and $\mathcal{D}$ are the lower block components of the $S O(3,3 \mid \mathbf{Z})$ transformation relating the original NCSYM gauge theory to the theory on the trivial bundle with $U(q)$ gauge group. Then one can make the following constant curvature connection and field redefinitions

$$
\begin{gathered}
\widehat{\nabla}^{i} \stackrel{\text { def }}{=}\left(H^{-1}\right)_{j}^{i} \nabla^{j}, \widehat{A}^{i} \stackrel{\text { def }}{=}\left(H^{-1}\right)_{j}^{i} A^{j}, \\
\widehat{D}^{i} \stackrel{\text { def }}{=}\left(H^{-1}\right)_{j}^{i} D^{j}, \\
\widehat{\mathcal{F}}^{k l}=\left[\widehat{\nabla}^{k}, \widehat{A}^{l}\right]-\left[\widehat{\nabla}^{l}, \widehat{A}^{k}\right]-i\left[\widehat{A}^{k}, \widehat{A}^{l}\right] .
\end{gathered}
$$

The curvature can be split into a constant term and a fluctuating piece

$$
\begin{gathered}
\mathcal{F}^{i j}=\mathcal{F}_{(0)}^{i j}+H_{k}^{i} H_{l}^{j} \widehat{\mathcal{F}}^{k l}, \\
\mathcal{F}^{0 k}=H_{l}^{k} \widehat{\mathcal{F}}^{0 l} .
\end{gathered}
$$

Using the matrices $Q$ and $R$ defined in [47] one can perform a change of integration variables $\widehat{\sigma}=\sigma Q R$, which introduces a Jacobian factor

$$
\int d^{3} \sigma \operatorname{tr} \Psi(\sigma)=\int d^{3} \widehat{\sigma} \operatorname{det}\left(Q^{-1}\right) \operatorname{tr} \Psi\left(\widehat{\sigma}(Q R)^{-1}\right)
$$

Making the substitutions (4.6), (4.7) and collecting similar terms one finds

$$
\mathcal{S}_{C S}=\frac{1}{2(2 \pi)^{2}} \int d t \int d^{3} \widehat{\sigma} \frac{q}{n} \operatorname{tr}\left(\varepsilon_{i j k}\left(\widehat{\mathcal{F}}^{0 i} A^{\prime j k}+2 \pi \widehat{\mathcal{F}}^{0 i} \widehat{\mathcal{F}}^{j k} A^{\prime}\right)\right)
$$


where

$$
\begin{gathered}
q A^{\prime}=\left(n \operatorname{det} Q^{-1} \operatorname{det} H\right) A \\
\varepsilon_{i j k} q A^{i j}=H_{k}^{l} \varepsilon_{i j l} n \operatorname{det} Q^{-1}\left(A^{i j}+2 \pi \mathcal{F}_{(0)}^{i j} A\right) .
\end{gathered}
$$

One can now rewrite the action in terms of new operators $\sigma_{i}^{\prime}, \partial^{\prime i}$, and $U^{\prime}$, and a $q$ dimensional trace. See [47] for a more detailed discussion of this substitution.

$$
\mathcal{S}_{C S}=\frac{1}{4(2 \pi)^{3}} \int \operatorname{tr}_{q}\left(2 \pi \mathcal{F}^{\prime} \wedge A^{\prime(2)}+\frac{1}{2} 2 \pi \mathcal{F}^{\prime} \wedge 2 \pi \mathcal{F}^{\prime} \wedge A^{\prime(0)}\right)
$$

More generally the action is invariant under duality transformations if the Chern-Simon parameters are related as follows

$$
\begin{gathered}
\bar{A}_{(0)}=|\operatorname{det}(\mathcal{C} \Theta+\mathcal{D})|^{-1 / 2} A_{(0)}, \\
*\left(\bar{A}^{(2)}+2 \pi \overline{\mathcal{F}}_{(0)} \bar{A}^{(0)}\right) \bar{n} \operatorname{det} \bar{Q}^{-1}=(\mathcal{C} \Theta+\mathcal{D})^{-T} *\left(A^{(2)}+2 \pi \mathcal{F}_{(0)} A^{(0)}\right) n \operatorname{det} Q^{-1},
\end{gathered}
$$

where $\mathcal{C}$ and $\mathcal{D}$ are the lower block components of the $S O(3,3 \mid \mathbf{Z})$ matrix relating the two theories, and the star operator is the duality operator acting only with respect to the compact coordinates.

Next we write the Chern-Simon parameters in term of the fields $C$ discussed in Section 4.1

$$
\begin{gathered}
A^{(0)}=*\left(C_{(3)}+C_{(1)} \wedge \Theta\right), \\
A^{(2)}=-* C_{(1)} .
\end{gathered}
$$

To obtain a compact form first define the column matrices $u$ and $v$ with components

$$
u_{i}=\frac{1}{2} M^{j k} C_{i j k}-n C_{i},
$$




$$
v^{i}=-M^{i j} C_{j}
$$

If $\chi$ transforms as a spinor, $u$ and $v$ are the block components of a $S O(3,3 \mid \mathbf{Z})$ vector as shown in the Appendix (B.8). Then using the identities (2.50) listed in Chapter 2, the transformation (4.8) can be written as

$$
\begin{gathered}
\bar{C}_{(3)}+\bar{C}_{(1)} \wedge \bar{\Theta}=|\operatorname{det}(\mathcal{C} \Theta+\mathcal{D})|^{-1 / 2}\left(C_{(3)}+C_{(1)} \wedge \Theta\right), \\
(\bar{u}-\bar{\Theta} \bar{v})=(\mathcal{C} \Theta+\mathcal{D})^{-T}(u-\Theta v) .
\end{gathered}
$$

Comparing (4.11) and (4.12) with (B.6) and (B.4) in Appendix B one sees that the R-R fields must transform in a spinor representation of $S O(3,3 \mid \mathbf{Z})$

$$
\bar{\chi}=S(\Lambda) \chi
$$

Thus the duality transformations of all the parameters of the NCSYM, including those of the Chern-Simon type terms, coincide with the transformation of moduli of the Type IIA strings compactified on a torus in the limit of vanishing $\alpha^{\prime}$ and $G_{i j}$.

Using the transformation properties of $g_{S Y M}^{-2}$ and $A^{(0)}$ it follows that the complex coupling

$$
\tau=A^{(0)}+\frac{4 \pi i}{g_{S Y M}^{2}}
$$

also transforms simply under the T-duality group with the same $\Theta$ dependent factor appearing in (4.8).

Finally note that the BPS spectrum corresponding to the electric fluxes ob- 
tained in [52] is modified in the presence of nonvanishing R-R moduli

$$
\begin{gathered}
\mathcal{E}^{U(n)}=\frac{g_{S}}{2}\left|n-\frac{1}{2} \operatorname{tr}(M \Theta)\right|^{-1} \\
\times\left(\left(n_{i}-u_{i}\right)-\Theta_{i k}\left(m^{k}-v^{k}\right)\right) G^{i j}\left(\left(n_{j}-u_{j}\right)-\Theta_{j l}\left(m^{l}-v^{l}\right)\right) .
\end{gathered}
$$

This result agrees with the small volume limit of the spectrum formula in [50] and reduces for vanishing $\Theta_{i j}$ to the result of [82, 81]. In [83] it was shown that shifts in the electric flux spectrum correspond to inequivalent geometric quantizations. These different quantizations are equivalent to the standard canonical quantization if one also includes topological terms in the action. 


\section{Chapter 5}

\section{T-duality of the BPS Spectrum}

As shown in Chapter 2, the action of a $U(n)$ NCSYM, with magnetic fluxes $M^{i j}$, can be written as the action of a $U(q)$ NCSYM on a trivial quantum bundle, where $q$ is the greatest common divisor of $n$ and $M^{i j}$. Thus these two theories must have identical spectra. The action contains magnetic backgrounds which we chose as in [47] so that the vacuum energy vanishes.

$$
\begin{aligned}
\mathcal{S}^{U(n)}= & \frac{1}{g_{S Y M}^{2}} \int d t \int d^{d} \sigma \sqrt{\operatorname{det}\left(G^{k l}\right)} \operatorname{tr}\left(\frac{1}{2} G_{i j} \mathcal{F}^{0 i} \mathcal{F}^{0 j}-\right. \\
& \frac{1}{4} G_{i j} G_{k l}\left(\mathcal{F}^{i k}-\mathcal{F}_{(0)}^{i k}\right)\left(\mathcal{F}^{j l}-\mathcal{F}_{(0)}^{j l}\right)+ \\
& \frac{1}{2} \sum_{a} \dot{X}^{a} \dot{X}^{a}-\frac{1}{2} \sum_{a} G_{i j}\left[D^{i}, X^{a}\right]\left[D^{j}, X^{a}\right]+ \\
& \left.\frac{1}{4} \sum_{a, b}\left[X^{a}, X^{b}\right]\left[X^{a}, X^{b}\right]+\text { fermions }\right) .
\end{aligned}
$$

All the equations in this chapter where $d$ is unspecified, are valid for the two and three dimensional case, but some may have to be modified in higher dimensions. For simplicity we will consider the case when $n$ and $M^{i j}$ are relatively prime. Then one can find a duality transformation $\Lambda$ such that $\bar{n}=1$ and $\bar{M}=0$ as was shown in [47]. From this point on, when we discuss the $U(n)$ theory we will use the the $d$-dimensional block matrices (2.42), with $\Lambda$ the particular transformation that takes the $U(n)$ theory into a $U(1)$ theory. For example the constant background 
field strength can be expressed in terms of the block components of $\Lambda$ as

$$
\mathcal{F}_{(0)}=\frac{-1}{2 \pi}(\mathcal{C} \Theta+\mathcal{D})^{-1} \mathcal{C}
$$

One can write the connection as a sum of a constant curvature $U(1)$ connection $\nabla_{i}$, a zero mode $A_{(0)}^{i}$, and a fluctuating part $A^{i}$

$$
\nabla^{i}-i A_{(0)}^{i} \mathbf{1}-i A^{i}\left(Z_{j}\right)=\partial^{i}+i F^{i j} \sigma_{j}-i A_{(0)}^{i} \mathbf{1}-i A^{i}\left(Z_{j}\right) .
$$

Note that $A^{i}$ does not contain the zero mode. The $Z_{i}$ 's are $n$-dimensional matrices which generate the algebra of adjoint sections. For example, in the two dimensional case one has $[49,27,36,40,47]$

$$
Z_{1}=e^{i \sigma_{i} Q_{1}^{i} / n} V^{b}, Z_{2}=e^{i \sigma_{i} Q_{2}^{i} / n} U,
$$

where $U$ and $V$ are the clock and shift matrices and $Q$ is a two dimensional matrix which reduces to the identity in the commutative case. Substituting this in the action gives

$$
\mathcal{S}^{U(n)}=\frac{1}{g_{S Y M}^{2}} \int d t \int d^{d} \sigma \sqrt{\operatorname{det}\left(G^{k l}\right)} \operatorname{tr} \frac{1}{2} G_{i j} \partial^{0} A_{(0)}^{i} \partial^{0} A_{(0)}^{j}+\ldots,
$$

where the dots stand for terms containing only $A^{i}$. Thus classically the zero modes decouple, and the action is just that of a free particle

$$
\mathcal{S}_{(0)}^{U(n)}=\int d t \frac{(2 \pi)^{2}}{2} \mathcal{M}_{i j} \dot{A}_{(0)}^{i} \dot{A}_{(0)}^{j},
$$

where the mass matrix is given by

$$
\mathcal{M}_{i j}=\left|n-\frac{1}{2} \operatorname{tr}(M \Theta)\right| \frac{(2 \pi)^{d-2} \sqrt{\operatorname{det}\left(G^{k l}\right)}}{g_{S Y M}^{2}} G_{i j} .
$$


In the commutative case the first factor on the right hand side of (5.3) reduces to $n$ and arises from taking the trace. The origin of this factor in the noncommutative case was discussed in $[27,47]$. The corresponding Hamiltonian is then ${ }^{1}$

$$
\mathcal{H}_{(0)}^{U(n)}=\frac{1}{2} \mathcal{M}^{i j} E_{i}^{(0)} E_{j}^{(0)},
$$

where $\mathcal{M}^{i j}$ is the inverse mass matrix and $E_{i}^{(0)}$ is the momentum conjugate to $A_{(0)}^{i}$

$$
E_{i}^{(0)}=\frac{1}{2 \pi i} \frac{\partial}{\partial A_{(0)}}
$$

Note that $E_{i}^{(0)}$ correspond to zero modes of the electric field.

\subsection{The $U(1)$ BPS Spectrum}

Before calculating the spectrum of (5.4) directly, we will use the duality invariance of the spectrum and obtain it by using the simpler dual $U(1)$ theory. We will use primes for all the variables in the $U(1)$ theory. In this case the mass matrix takes the form

$$
\mathcal{M}_{i j}^{\prime}=\frac{(2 \pi)^{d-2} \sqrt{\operatorname{det}\left(G^{\prime k l}\right)}}{g^{\prime 2}} G_{i j M}^{\prime} .
$$

Just as in the commutative $U(1)$ supersymmetric gauge theory [84] the zero modes live on a torus. To see this consider the gauge transformations

$$
U_{i}^{\prime}=e^{i \sigma_{i}^{\prime}}
$$

These gauge transformations are single valued and leave the trivial transition functions invariant. Under these gauge transformations the connection transforms

\footnotetext{
${ }^{1}$ This only includes the energy coming form the electric zero modes.
} 
as

$$
U_{j}^{\prime-1}\left(\partial^{\prime i}-i A_{(0)}^{\prime i}-i A^{\prime i}\left(U_{k}^{\prime}\right)\right) U_{j}^{\prime}=\partial^{\prime i}-i\left(A_{(0)}^{\prime i}-\delta_{j}^{i}\right)-i A^{\prime i}\left(e^{-2 \pi i \Theta_{j k}^{\prime}} U_{k}^{\prime}\right) .
$$

For vanishing $\Theta^{\prime}$ the effect of these gauge transformations is just a shift of the zero mode and one has the following gauge equivalences ${A^{\prime}}_{(0)}^{i} \sim{A^{\prime}}_{(0)}^{i}+\delta_{j}^{i}$. Note that $\delta_{(j)}^{i}$ for $j=1, \ldots, d$ form a basis for a lattice $L^{\prime}$ and the configuration space is $\mathbf{R}^{d} / L^{\prime}$. The conjugate momenta are then quantized

$$
E_{i}^{\prime(0)}=n_{i}^{\prime}
$$

and the spectrum of zero modes is then given by

$$
\mathcal{E}^{U(1)}=\frac{1}{2} \mathcal{M}^{\prime i j} n_{i}^{\prime} n_{j}^{\prime}
$$

However in the noncommutative case one can see that the above gauge transformations also produces a translation in the $k$ direction proportional to $\Theta_{j k}^{\prime}$. This results in a modification of the spectrum similar to the Witten-Olive effect [53]. Let as define the total momentum operator operators $P_{i}^{\prime}$ such that

$$
\left[P^{\prime i}, \Psi\right]=-i \partial^{\prime i} \Psi
$$

where $\Psi$ is an arbitrary field of the theory. The momentum $P^{\prime i}$ defined by $(5.6)$ is not the standard gauge invariant total momentum but the difference between the two is the generator of a gauge transformation with the gauge parameter equal to the $i$-component of the gauge field. Thus on gauge invariant states the total momentum defined above and the gauge invariant momentum have the same effect. 
The operator generating the gauge transformation is $[50,51]$

$$
\exp \left(2 \pi i\left(E_{j}^{\prime(0)}+\Theta_{j k}^{\prime} P^{\prime k}\right)\right)
$$

Translation by an integral number of periods on a trivial bundle must leave the physical system invariant. The operators generating these translations are given by

$$
\exp \left(2 \pi i P^{\prime k}\right)
$$

The operators (5.7) and (5.8) act as the identity on physical states so one obtains the quantization

$$
E_{j}^{(0)}+\Theta_{j k}^{\prime} P^{\prime k}=n_{j}^{\prime}, \quad P^{\prime j}=m^{\prime j},
$$

where $n_{j}$ and $m^{j}$ are integers. The spectrum of zero modes is then given by

$$
\mathcal{E}^{U(1)}=\frac{1}{2} \mathcal{M}^{\prime i j}\left(n_{i}^{\prime}-\Theta_{i k}^{\prime} m^{\prime k}\right)\left(n_{j}^{\prime}-\Theta_{j l}^{\prime} m^{\prime l}\right)
$$

This result has the following geometric interpretation. In the sectors of nonvanishing momentum the wave function for the zero modes is not strictly speaking a function but rather a section on a twisted bundle over the torus $\mathbf{R}^{d} / L^{\prime}$ with twists given by $\exp \left(\Theta_{i k}^{\prime} m^{\prime k}\right)$.

\subsection{Dual $U(n)$ BPS Spectrum}

Using the duality transformations (1.5) we can express the spectrum in terms of the $U(n)$ parameters

$$
\mathcal{E}^{U(n)}=\frac{1}{2} \frac{g_{S Y M}^{2}}{(2 \pi)^{d-2} \sqrt{\operatorname{det}\left(G^{i j}\right)}}|\operatorname{det}(\mathcal{C} \Theta+\mathcal{D})|^{-1 / 2}
$$




$$
\times G^{i j}\left(n_{i}-\Theta_{i k} m^{k}\right)\left(n_{j}-\Theta_{j k} m^{k}\right),
$$

where we also performed a duality transformation on the quantum numbers $[50,51]$

$$
\left(\begin{array}{c}
n_{i}^{\prime} \\
m^{\prime i}
\end{array}\right)=\left(\begin{array}{ll}
\mathcal{A} & \mathcal{B} \\
\mathcal{C} & \mathcal{D}
\end{array}\right)\left(\begin{array}{c}
n_{i} \\
m^{i}
\end{array}\right)
$$

Next consider in more detail the two dimensional case. The parameters of the $U(1)$ and $U(n)$ NCSYM are related by the $S O(2,2 \mid \mathbf{Z})$ transformation [47]

$$
\Lambda=\left(\begin{array}{cc}
a I_{2} & b \varepsilon \\
-m \varepsilon & n I_{2}
\end{array}\right)
$$

where $\varepsilon$ is a two dimensional matrix with the only nonvanishing entries given by $\varepsilon_{12}=-\varepsilon_{21}=1$. In this case $(\mathcal{C} \Theta+\mathcal{D})_{j}^{i}=(n+\theta m) \delta_{j}^{i}$ and the spectrum is

$$
\mathcal{E}^{U(n)}=\frac{1}{2} \frac{g_{S Y M}^{2}}{(2 \pi)^{d-2}|n+\theta m| \sqrt{\operatorname{det}\left(G^{k l}\right)}} G^{i j}\left(n_{i}+\theta m_{i}\right)\left(n_{j}+\theta m_{j}\right),
$$

where $m^{i}=\varepsilon^{i j} m_{j}$. This result ${ }^{2}$ has the expected factor of $|n+\theta m|$ in the denominator. In the DLCQ formulation of M theory this factor is proportional to the kinetic momentum in the compact light-like direction and is expected to appear in the denominator of the DLCQ Hamiltonian.

Next the spectrum is obtained directly in the $U(n)$ theory. We will do this in two ways. First, consider the generators of the adjoint algebra, the $Z_{i}$ 's. These generators satisfy

$$
Z_{k}\left(\sigma_{i}+2 \pi \delta_{i}^{j}\right)=\Omega_{j} Z_{k}\left(\sigma_{i}\right) \Omega_{j}^{-1}
$$

\footnotetext{
${ }^{2}$ Expressed in terms of the string coupling constant of the auxiliary string theory the spectrum takes the simpler form $E^{U(n)}=\frac{1}{2} g_{s}|n+\theta m|^{-1} G^{k l}\left(n_{k}+\theta m_{k}\right)\left(n_{l}+\theta m_{l}\right)$.
} 
Besides having the privileged role of generators for the sections of the adjoint bundle, the $Z_{i}$ 's can also be used to perform gauge transformations since they are unitary. Next, rewrite (5.12) as

$$
\Omega_{j}=Z_{k}\left(\sigma_{i}+2 \pi \delta_{i}^{j}\right)^{-1} \Omega_{j} Z_{k}\left(\sigma_{i}\right)
$$

The right hand side of (5.13) gives the transformation of the transition functions under the $Z_{i}$ gauge transformation. One can see that, just as in the $U(1)$ case where the gauge transformations $U_{i}^{\prime}$ left the transition functions trivial, the $Z_{i}^{\prime}$ s leave the transition functions invariant. Following the same strategy as in the $U(1)$ case, where we used the $U_{i}^{\prime}$ to find the configuration space of the zero modes, we will use here $Z_{i}$

$$
\begin{gathered}
Z_{j}^{-1}\left(\nabla^{i}-i A_{(0)}^{i} \mathbf{1}-i A^{i}\left(Z_{k}\right)\right) Z_{j}= \\
\nabla^{i}-i\left(A_{(0)}^{i}-\left((\mathcal{C} \Theta+\mathcal{D})^{-1}\right)_{j}^{i}\right) \mathbf{1}-i A^{i}\left(e^{-2 \pi i \Theta_{j k}^{\prime}} Z_{k}\right) .
\end{gathered}
$$

Note that again we have separated the zero mode of the gauge connection and we have used the identity [47]

$$
\left[\nabla^{i}, Z_{j}\right]=i\left((\mathcal{C} \Theta+\mathcal{D})^{-1}\right)_{j}^{i} Z_{j}
$$

One can express the gauge transformed connection as

$$
e^{-2 \pi(\mathcal{A} \Theta+\mathcal{B})_{j k} \nabla^{k}}\left(\nabla^{i}-i A_{(0)}^{i} \mathbf{1}-i A^{i}\left(Z_{k}\right)\right) e^{2 \pi(\mathcal{A} \Theta+\mathcal{B})_{j k} \nabla^{k}}+i \mathcal{A}_{j}^{i} \mathbf{1},
$$

where we used

$$
(\mathcal{C} \Theta+\mathcal{D})^{-1}=\left(\mathcal{A}-\Theta^{\prime} \mathcal{C}\right)^{T}
$$


and (5.2) to rewrite the extra shift in the zero mode.

Next we define the momentum operator by its action on the fields of the theory. For example on the gauge fields $P^{i}$ acts as

$$
\left[P^{i}, A_{(0)}^{j} \mathbf{1}+A^{j}\left(Z_{k}\right)\right]=-i\left[\nabla^{i}, A^{j}\left(Z_{k}\right)\right]-i \mathcal{F}_{(0)}^{i j} .
$$

Note that $P^{i}$ also acts on the zero mode $A_{(0)}^{i}$. This can be understood as follows. When defining the momentum there is a choice whether to include as part of the system the magnetic background $\mathcal{F}_{(0)}^{i j}$. The standard gauge invariant momentum for which the momentum density is $\operatorname{tr}\left(F^{i j} E_{j}\right)$ can be written as the sum of two terms. The first is just the momentum translating the part of the system that does not include the magnetic background and whose momentum density is $\operatorname{tr}\left(\left(F^{i j}-\right.\right.$ $\left.\left.F_{(0)}^{i j}\right) E_{j}\right)$. The second term is an operator shifting the zero mode of the gauge field as in (5.15). Then our $P^{i}$ can be identified, up to the generator of a gauge transformation, with the total momentum that includes the magnetic background. Furthermore, one can identify, up to the generator of a gauge transformation, the first term on the right hand side of (5.15) as the action of the momentum operator that translates only the fluctuating part. As will be seen later it is the momentum whose density is $\operatorname{tr}\left(F^{i j} E_{j}\right)$ that appears in the $S O(d, d \mid \mathbf{Z})$ duality transformation. A convenient way of writing the action of $P^{i}$ on the gauge field is

$$
\left[P^{i},-i A_{(0)}^{j} \mathbf{1}-i A^{j}\left(Z_{k}\right)\right]=-i\left[\nabla^{i}, \nabla^{j}-i A_{(0)}^{j} \mathbf{1}-i A^{j}\left(Z_{k}\right)\right]
$$

Then using (5.14) one sees that the quantum operator which implements the gauge 
transformation above is given by

$$
\exp \left(2 \pi i\left(\mathcal{A}_{j}^{k}\left(E_{k}^{(0)}+\Theta_{k l} P^{l}\right)+\mathcal{B}_{j k} P^{k}\right)\right)
$$

The momentum operator $P^{i}$ has integer eigenvalues since the space is a torus with lengths $2 \pi$. One can also see this by considering the operator

$$
\exp \left(2 \pi i\left(\mathcal{C}^{j i} E_{i}^{(0)}+\left(\mathcal{C}^{j k} \Theta_{k i}+\mathcal{D}_{i}^{j}\right) P^{i}\right)\right)
$$

This acts trivially on every operator in the $U(n)$ theory. In particular the combination of operators in the exponent has no effect on the zero mode. The condition that (5.16) and (5.17) should act as the identity on the physical Hilbert space is equivalent to the quantization

$$
\begin{aligned}
& \mathcal{A}_{j}^{k}\left(E_{k}^{(0)}+\Theta_{k l} P^{l}\right)+\mathcal{B}_{j k} P^{k}=n_{j}^{\prime}, \\
& \mathcal{C}^{j k}\left(E_{k}^{(0)}+\Theta_{k l} P^{l}\right)+\mathcal{D}_{k}^{j} P^{k}=m^{\prime j} .
\end{aligned}
$$

Since the matrices $\mathcal{A}, \mathcal{B}, \mathcal{C}$, and $\mathcal{D}$ are the block components of an element of $S O(d, d \mid \mathbf{Z})$ this is equivalent to

$$
E_{j}^{(0)}+\Theta_{j k} P^{k}=n_{j}, \quad P^{j}=m^{j},
$$

where $n_{j}$ and $m^{j}$ are integers. Using the Hamiltonian (5.4) and the above quantization the electric flux spectrum is

$$
\begin{aligned}
\mathcal{E}^{U(n)}= & \frac{1}{2} \frac{g_{S Y M}^{2}}{(2 \pi)^{d-2} \sqrt{\operatorname{det}\left(G^{i j}\right)}}\left|n-\frac{1}{2} \operatorname{tr}(M \Theta)\right|^{-1} \\
& \times G^{i j}\left(n_{i}-\Theta_{i k} m^{k}\right)\left(n_{j}-\Theta_{j k} m^{k}\right)
\end{aligned}
$$


which is identical to the result (5.9) obtained by duality.

Finally we present an alternative derivation of the spectrum using the gauge transformations $\exp \left(i \bar{\sigma}_{i}\right)$ where $\bar{\sigma}_{i}=\sigma_{j} Q_{i}^{j}$ and $Q_{i}^{j}$ is a matrix defined in [47] and equals the identity for vanishing deformation parameter or magnetic background. This derivation is closely related to the derivation of the spectrum in $[50,51]$. As discussed in [47] gauge invariant quantities such as the Lagrangian density have periodicity $2 \pi$ in the $\bar{\sigma}_{i}$ variables. Then one can use $\bar{U}_{i}=\exp \left(i \bar{\sigma}_{i}\right)$ as a gauge transformation just as one used $U_{i}^{\prime}$ in the $U(1)$ theory. Note first that $\bar{U}_{i}$ is a globally defined gauge transformation. It is convenient to write it as $\bar{U}_{i}=\mathcal{U}_{i} e^{2 \pi \Theta_{i j} \nabla^{j}}$. Here $\mathcal{U}_{i}=e^{i \sigma_{i}-2 \pi \Theta_{i j} \partial^{j}}$ and is the variable implementing the quotient condition [27]. The effect of this gauge transformation is

$$
\begin{gathered}
\bar{U}_{j}^{-1}\left(\nabla^{i}-i A_{(0)}^{i} \mathbf{1}-i A^{i}\left(Z_{k}\right)\right) \bar{U}_{j}= \\
e^{-2 \pi \Theta_{i j} \nabla^{j}}\left(\nabla^{i}-i A_{(0)}^{i} \mathbf{1}-i A^{i}\left(Z_{k}\right)\right) e^{2 \pi \Theta_{i j} \nabla^{j}}+i \delta_{j}^{i} .
\end{gathered}
$$

The operator implementing this gauge transformation in the Hilbert space is

$$
\exp \left(-2 \pi i\left(E_{k}^{(0)}+\Theta_{k l} P^{l}\right)\right)
$$

Again, on gauge invariant states this operator acts trivially and together with the quantization of the momentum results in the same spectrum (5.18) as using $Z_{i}$. Note that the second method of deriving the $U(n)$ spectrum is similar in spirit to the derivation of the $U(1)$ spectrum. For example the gauge transformation is an element of the $U(1)$ subgroup. However, the first derivation is instructive since it exhibits inside the $U(n)$ theory the dual $U(1)$ theory variables such as $P^{\prime i}$ and $E_{i}^{\prime}$. 


\section{Chapter 6}

\section{Duality Invariance}

In the remainder of the thesis, we will be discussing self-duality of the type first noticed in the Maxwell electromagnetic theory, which is invariant when one replaces the field strength with its Hodge dual. Very few theories with this type of invariance are known. The $U(1)$ Born-Infeld is one example. Although in certain limits it reduces to the Maxwell theory, the Born-Infeld theory is non-linear. We will, in fact, find a number of generalizations of this theory which possess nonlinear self-duality. In this chapter, we describe how the theory of self-duality introduced in $[3,4]$ is modified when we consider complex abelian gauge fields.

We begin by considering a linear action of the duality group which mixes the field strengths and their duals but not their complex conjugates. We will refer to this as a holomorphic action. Under these conditions, the largest allowed duality group is $U(n, n)$ where $n$ is the number of complex gauge fields. If we do not require a holomorphic action, $n$ complex gauge fields are equivalent to $2 n$ real gauge fields in which case the largest possible duality group is $S p(4 n, \mathbf{R})$. Later, in Section 8.1, we will also introduce a Born-Infeld action with real gauge fields which we show to have the largest allowed duality group given the number of gauge

fields. However, the arguments leading to this involve Lagrangians with complex 
gauge fields.

Consider a theory of $n$ complex abelian gauge fields and a scalar field $S$ which is an $n$-dimensional complex matrix. Here we do not require $S$ to be symmetric and as a result we find a larger duality group than the one appearing in [54]. The gauge fields only enter in the Lagrangian through their field strengths $F^{a}$, where $a=1, \ldots, n$, and their complex conjugates $\bar{F}^{a}$

$$
L=L\left(F^{a}, \bar{F}^{a}, S, \ldots\right)
$$

The dots in (6.1) represent possible auxiliary fields which could also be present in $L$. As we will show later, with the scalar field $S$ present the duality group is noncompact while without the scalar field only the maximal compact subgroup survives. We can also add to this Lagrangian a kinetic term for the scalar field $S$. As explained in [3] additional physical fields, e.g. spinors, can also be introduced, but we shall not consider them explicitly in this paper except in Section 8.2 where the supersymmetric Born-Infeld theory is discussed.

The dual field strength, or rather the Hodge dual of the dual field strength, $\widetilde{G}_{\mu \nu}^{a}=\frac{1}{2} \varepsilon_{\mu \nu \rho \sigma} G^{a \rho \sigma}$, is defined as

$$
\tilde{G}_{\mu \nu}^{a} \equiv 2 \frac{\partial L}{\partial \bar{F}^{a \mu \nu}}, \quad \tilde{\bar{G}}_{\mu \nu}^{a} \equiv 2 \frac{\partial L}{\partial F^{a \mu \nu}}
$$

Throughout this paper we will assume that we are in four space-time dimensions, except in Section 8.3, where we will show how to generalize our results to theories in even space-time dimensions.

The equations of motion and Bianchi identities transform covariantly under 
the following holomorphic infinitesimal transformations

$$
\delta\left(\begin{array}{l}
G \\
F
\end{array}\right)=\left(\begin{array}{ll}
A & B \\
C & D
\end{array}\right)\left(\begin{array}{l}
G \\
F
\end{array}\right) .
$$

Let $\phi$ denote all the scalar fields appearing in the Lagrangian and $\phi_{\mu}=\partial_{\mu} \phi$. The infinitesimal transformations of the scalar fields are given by

$$
\delta \phi^{i}=\xi^{i}(\phi)
$$

where $\xi^{i}$ are components of a vector field on the scalar field space. The most general Lagrangian, neglecting possible fermionic fields, has the form $L\left(F, \bar{F}, \phi, \phi_{\mu}\right)$. Its variation under $(6.3)(6.4)$ can be written as

$$
\delta L=\left[\delta_{\phi}+\left(G C^{T}+F D^{T}\right) \frac{\partial}{\partial F}+\left(\bar{G} C^{\dagger}+\bar{F} D^{\dagger}\right) \frac{\partial}{\partial \bar{F}}\right] L,
$$

where $\delta_{\phi} L$ is given by

$$
\delta_{\phi} L=\left(\xi^{i} \frac{\partial}{\partial \phi^{i}}+\phi_{\mu}^{j} \frac{\partial \xi^{i}}{\partial \phi^{j}} \frac{\partial}{\partial \phi_{\mu}^{i}}\right) L
$$

The variation of the Lagrangian must satisfy certain consistency conditions. First note that

$$
\frac{\partial}{\partial F}(\delta L)=\delta\left(\frac{\partial L}{\partial F}\right)+\frac{\partial G}{\partial F} C^{T} \frac{\partial L}{\partial F}+D^{T} \frac{\partial L}{\partial F}+\frac{\partial \bar{G}}{\partial F} C^{\dagger} \frac{\partial L}{\partial \bar{F}}
$$

Using (6.2) we obtain

$$
\delta \widetilde{\bar{G}}=2 \frac{\partial}{\partial F}(\delta L)-\bar{G} C \frac{\partial \widetilde{G}}{\partial F}-\frac{\partial \bar{G}}{\partial F} C^{\dagger} \widetilde{G}-\widetilde{\bar{G}} D
$$

and this should be consistent with the variation obtained from (6.3)

$$
\delta \widetilde{\bar{G}}=\widetilde{\bar{G}} A^{\dagger}+\widetilde{\bar{F}} B^{\dagger}
$$


Equating (6.5) and (6.6) we obtain the consistency condition

$$
\begin{gathered}
\frac{\partial}{\partial F}\left(\delta L-\frac{1}{4} \bar{G}\left(C^{\dagger}+C\right) \widetilde{G}-\frac{1}{4} \bar{F}\left(B^{\dagger}+B\right) \widetilde{F}\right)= \\
\frac{\partial L}{\partial F}\left(D+A^{\dagger}\right)+\frac{1}{4} \bar{G}\left(C-C^{\dagger}\right) \frac{\partial \widetilde{G}}{\partial F}-\frac{1}{4} \frac{\partial \bar{G}}{\partial F}\left(C-C^{\dagger}\right) G+\frac{1}{4} \widetilde{\bar{F}}\left(B^{\dagger}-B\right) .
\end{gathered}
$$

The right hand side of the above equation must be a total derivative since the left hand side is one. This is possible if

$$
A^{\dagger}+D=\varepsilon I, \quad B^{\dagger}=B, \quad C^{\dagger}=C,
$$

where $\varepsilon$ is a real parameter. These are the relations of the fundamental representation of the $U(n, n) \times \mathbb{R}^{\star}$ Lie algebra ${ }^{1}$. We will only consider the case when $\varepsilon$ vanishes. Thus we assume

$$
A^{\dagger}=-D, \quad B^{\dagger}=B, \quad C^{\dagger}=C
$$

The relations (6.8) define the fundamental representation of the Lie algebra of $U(n, n)$. However, in general the transformations (6.4) of the scalar fields can be implemented only for a subgroup $H$ of $U(n, n)$. The duality group $H$ depends both on the field content and the nature of the interactions of the scalar fields.

Using (6.8) the consistency condition (6.7) can be written as

$$
\frac{\partial}{\partial F}\left(\delta L-\frac{1}{2} \bar{F} B \widetilde{F}-\frac{1}{2} \bar{G} C \widetilde{G}\right)=0
$$

Another consistency condition is obtained by applying the Euler operator

$$
\frac{\partial}{\partial \phi^{i}}-\partial_{\mu} \frac{\partial}{\partial \phi_{\mu}^{i}}
$$

\footnotetext{
${ }^{1} \mathbb{R}^{\star}$ denotes the group of nonvanishing real numbers.
} 
on the variation of the Lagrangian. Just as in the derivation of [3], by assuming (6.8) we obtain

$$
\left(\frac{\partial}{\partial \phi^{i}}-\partial_{\mu} \frac{\partial}{\partial \phi_{\mu}^{i}}\right)\left(\delta L-\frac{1}{2} \bar{F} B \widetilde{F}-\frac{1}{2} \bar{G} C \widetilde{G}\right)=\delta E_{i}+\frac{\partial \xi^{j}}{\partial \phi^{i}} E_{j}
$$

where $E_{i}$ is the left hand side of the equation of motion for the field $\phi^{i}$

$$
E_{i}=\frac{\partial L}{\partial \phi^{i}}-\partial_{\mu} \frac{\partial L}{\partial \phi_{\mu}^{i}}
$$

A sufficient condition to satisfy the consistency equation (6.9) is given by

$$
\delta L=\frac{1}{2}(\bar{F} B \widetilde{F}+\bar{G} C \widetilde{G})
$$

This is equivalent to the invariance of the following combination

$$
L-\frac{1}{4} \bar{F} \widetilde{G}-\frac{1}{4} F \widetilde{\bar{G}}
$$

Using (6.11) in (6.10) we obtain

$$
\delta E_{i}=-\frac{\partial \xi^{j}}{\partial \phi^{i}} E_{j}
$$

showing that the equations of motion for the scalar fields form a multiplet under the duality group $H$. In the examples discussed in this paper the duality group will be $U(n, n)$ for complex gauge fields and $S p(2 n, \mathbb{R})$ for real gauge fields. Ignoring a possible $\mathbb{R}^{\star}$ factor, present only for a nonvanishing $\varepsilon$, we will refer to these as the maximal noncompact duality groups.

The corresponding finite duality transformations are given by

$$
\left(\begin{array}{c}
G^{\prime} \\
F^{\prime}
\end{array}\right)=M\left(\begin{array}{c}
G \\
F
\end{array}\right)
$$


Here $M$ is an $U(n, n)$ matrix satisfying

$$
M^{\dagger} \mathbb{K} M=\mathbb{K},
$$

where $M$ and $\mathbb{K}$ have the block form

$$
M=\left(\begin{array}{ll}
a & b \\
c & d
\end{array}\right), \quad \mathbb{K}=\left(\begin{array}{rr}
0 & 1 \\
-1 & 0
\end{array}\right) .
$$

Note that the invariant $\mathbb{K}$ defining $U(n, n)$ is the usual off diagonal symplectic form. This explains the similarity of our results with the real case discussed in [3]. One can check that (6.15) implies the following relations for the block components of $M$

$$
c^{\dagger} a=a^{\dagger} c, \quad b^{\dagger} d=d^{\dagger} b, \quad d^{\dagger} a-b^{\dagger} c=1 .
$$

The infinitesimal relations (6.8) can be obtained from the finite relations (6.16) using

$$
a \approx 1+A, \quad b \approx B, \quad c \approx C, d \approx 1+D .
$$

In much of this paper we consider Lagrangians which do not depend on the scalar field $S$, i.e. they depend only on the gauge field strengths and perhaps auxiliary scalar fields, and are invariant only under the maximal compact subgroup $U(n) \times U(n)$ of $U(n, n)$. Then there is a way to introduce the scalar field $S$ which extends the duality group to $U(n, n)$. The maximal compact subgroup $U(n) \times U(n)$ is the subgroup of $U(n, n)$ obtained by requiring (6.16) and

$$
a=d, \quad b=-c .
$$


The corresponding infinitesimal relations are (6.8) and

$$
A=D, \quad B=-C
$$

Let $\mathcal{L}(F, \bar{F})$ be a Lagrangian describing a theory invariant under $U(n) \times U(n)$, where we suppress the dependence on the auxiliary fields. Then we define a new Lagrangian

$$
L\left(F, \bar{F}, S_{1}, R, R^{\dagger}\right) \equiv \mathcal{L}\left(R F, \bar{F} R^{\dagger}\right)+\frac{1}{2} \operatorname{Tr}\left(S_{1} \tilde{F} \bar{F}\right)
$$

where $S_{1}$ is a hermitian $n$-dimensional matrix and $R$ is a nondegenerate $n$ dimensional matrix. This Lagrangian describes a theory invariant under $U(n, n)$ if we transform the scalar fields $S_{1}$ and $R$ as discussed below. As we will see, the duality invariance of the theory described by $\mathcal{L}$ implies that $L$ depends on $R$ and $R^{\dagger}$ only through the hermitian positive definite matrix

$$
S_{2}=R^{\dagger} R
$$

We also define $S \equiv S_{1}+i S_{2}$. Under the duality group $S$ transforms by fractional transformation

$$
S^{\prime}=(a S+b)(c S+d)^{-1}
$$

whose infinitesimal form is

$$
\delta S=B+A S-S D-S C S .
$$

It is also convenient to write down the transformation of $S_{2}$

$$
S_{2}^{\prime}=(c S+d)^{-\dagger} S_{2}(c S+d)^{-1} .
$$


In (6.21) and below we use the notation $-\dagger$ for the hermitian conjugate of the inverse.

Next we show that the Lagrangian $L$ defined in (6.17) corresponds to a $U(n, n)$ duality invariant theory. We follow closely [64] where the case of real gauge fields was considered. The proof in [64] generalizes the introduction of a single complex scalar field for a $U(1)$ interacting theory discussed in $[9,10]$. Using the fact that $\mathcal{L}(F, \bar{F})$ satisfies $(6.11)$ with compact duality rotations we have

$$
\begin{gathered}
\bar{G}^{a} \widetilde{G}^{b}+\bar{F}^{a} \widetilde{F}^{b}=0, \\
\bar{G}^{a} \widetilde{F}^{b}-\bar{F}^{a} \widetilde{G}^{b}=0 .
\end{gathered}
$$

The relation (6.22) corresponds to transformations with $A=0$ while (6.23) is obtained by setting $C=0$. We now introduce some convenient notation

$$
\mathcal{F}=R F, \quad \widetilde{\mathcal{G}}=2 \frac{\partial \mathcal{L}(\mathcal{F}, \overline{\mathcal{F}})}{\partial \mathcal{F}}
$$

Given a Lagrangian $\mathcal{L}$ which depends on $F$ but not its derivatives, we may rewrite $(6.22)$ and $(6.23)$ as

$$
\begin{aligned}
& \overline{\mathcal{G}}^{a} \widetilde{\mathcal{G}}^{b}+\overline{\mathcal{F}}^{a} \widetilde{\mathcal{F}}^{b}=0, \\
& \overline{\mathcal{G}}^{a} \widetilde{\mathcal{F}}^{b}-\overline{\mathcal{F}}^{a} \widetilde{\mathcal{G}}^{b}=0 .
\end{aligned}
$$

We would like to show that under an infinitesimal $U(n, n)$ duality transformation the change in the Lagrangian $L$ defined in (6.17) satisfies the duality condition $(6.11)$

$$
\left(\delta_{F}+\delta_{\bar{F}}+\delta_{S_{1}}+\delta_{R}+\delta_{R^{\dagger}}\right) L=\frac{1}{2}(\bar{F} B \widetilde{F}+\bar{G} C \widetilde{G})
$$


A transformation law for $R$ which is consistent with the relation $R^{\dagger} R=S_{2}$ and the duality transformation (6.21) of $S_{2}$ is given by

$$
R^{\prime}=R(c S+d)^{-1}
$$

whose infinitesimal transformation is $\delta R=-R(C S+D)$. This choice is somewhat arbitrary since equation (6.23) is equivalent to the Lagrangian $\mathcal{L}$ being invariant under left multiplication of the gauge field strength by unitary matrices $U$

$$
\mathcal{L}\left(U F, \bar{F} U^{\dagger}\right)=\mathcal{L}(F, \bar{F})
$$

This ensures that left multiplication of $R$ by a unitary matrix leaves the Lagrangian $L$ invariant. It follows that the Lagrangian $L$ only depends on $S_{2}$ and not on the specific $R$ chosen $^{2}$, as we have already mentioned. Any variation of the form $\delta R=\Omega R-R(C S+D)$, where $\Omega$ is anti-hermitian, would still preserve the relation $R^{\dagger} R=S_{2}$

Using the above transformation of $R$ one can show that (6.27) is equivalent to the vanishing of the following expression

$$
\begin{gathered}
\widetilde{G}^{a} \bar{G}^{b}-\widetilde{G}^{a}\left(\bar{F} S_{1}\right)^{b}-\left(S_{1} \widetilde{F}\right)^{a} \bar{G}^{b}+\left(S_{1} \widetilde{F}\right)^{a}\left(\bar{F} S_{1}\right)^{b}+\left(S_{2} \widetilde{F}\right)^{a}\left(\bar{F} S_{2}\right)^{b}+ \\
-i\left(\left(S_{2} \widetilde{F}\right)^{a} \bar{G}^{b}-\left(S_{2} \widetilde{F}\right)^{a}\left(\bar{F} S_{1}\right)^{b}-\widetilde{G}^{a}\left(\bar{F} S_{2}\right)^{b}+\left(S_{1} \widetilde{F}\right)^{a}\left(\bar{F} S_{2}\right)^{b}\right)
\end{gathered}
$$

Using the relation $\mathcal{G}=R^{-\dagger}\left(G-S_{1} F\right)$, which follows from (6.2) and (6.24), the first and second lines of this expression are equivalent to the left hand side of (6.25)

\footnotetext{
${ }^{2}$ Note that $S_{2}$ is a positive definite hermitian metric and $R$ is a vielbein. The Lagrangian only depends on the metric and the arbitrariness in the choice of vielbein introduces a $U(n)$ gauge invariance.
} 
and (6.26) respectively. Thus (6.27) is satisfied concluding the proof that the theory with the Lagrangian $L$ is invariant under $U(n, n)$.

Conversely, if we are given a Lagrangian $L$ with equations of motion invariant under $U(n, n)$ we can obtain a theory without the scalar field $S$ by setting $S=$ $i$. Then the duality group is broken to the stability group of $S=i$ which is $U(n) \times U(n)$, the maximal compact subgroup. Thus we can easily move between the theory with a scalar field $S$ and the theory without $S$.

We also give the infinitesimal transformation of $\mathcal{F}$ and $\mathcal{G}$

$$
\begin{aligned}
\delta \mathcal{G} & =R C R^{\dagger} \mathcal{G}-i R C R^{\dagger} \mathcal{F} \\
\delta \mathcal{F} & =-R C R^{\dagger} \mathcal{F}-i R C R^{\dagger} \mathcal{G}
\end{aligned}
$$

The last term in (6.28) is a unitary transformation and could be canceled by using a different choice for the transformation of $R$. The first term is an infinitesimal duality transformation belonging to the maximal compact subgroup $U(n) \times U(n)$. Note however that it is a space-time dependent duality transformation.

Next we find the differential equation that a Lagrangian must satisfy if the equations of motion are invariant under the maximal compact duality group. We are therefore considering a Lagrangian without the scalar field $S$. We will also assume that the auxiliary fields have been eliminated, the field strengths appear in the Lagrangian only through the Lorentz invariant combinations

$$
\alpha^{a b} \equiv \frac{1}{2} F^{a} \bar{F}^{b}, \quad \beta^{a b} \equiv \frac{1}{2} \widetilde{F}^{a} \bar{F}^{b},
$$

and that the Lagrangian is a sum of traces (or of products of traces) of monomials 
in $\alpha$ and $\beta$. If the Lagrangian has such a form, equation (6.23) is satisfied. Then under a compact duality transformation the variation of the Lagrangian is

$$
\delta \mathcal{L}=\operatorname{Tr}\left(\mathcal{L}_{\alpha} \delta \alpha+\mathcal{L}_{\beta} \delta \beta\right)
$$

where we define

$$
\mathcal{L}_{\alpha} \equiv \frac{\partial \mathcal{L}}{\partial \alpha^{T}}, \quad \mathcal{L}_{\beta} \equiv \frac{\partial \mathcal{L}}{\partial \beta^{T}}
$$

Using the definitions (6.2) and (6.29), we find that (6.22) is equivalent to

$$
\mathcal{L}_{\beta} \beta \mathcal{L}_{\beta}-\mathcal{L}_{\alpha} \beta \mathcal{L}_{\alpha}+\mathcal{L}_{\alpha} \alpha \mathcal{L}_{\beta}+\mathcal{L}_{\beta} \alpha \mathcal{L}_{\alpha}+\beta=0
$$

This is a generalization of the differential equation introduced in [10] where the case of a single real gauge field was considered. Equation (6.30) is invariant under the following transformation

$$
\begin{aligned}
& \alpha^{\prime}=\alpha, \\
& \beta^{\prime}=-\beta .
\end{aligned}
$$

If one considers a self-dual theory with $n$ real field strengths $F_{R}$, where now $\alpha$ and $\beta$ are defined by $\alpha^{a b}=1 / 4 F_{R}^{a} F_{R}^{b}$ and $\beta^{a b}=1 / 4 F_{R}^{a} \tilde{F}_{R}^{b}$, equation (6.30) still holds. In this case one can extend the duality group from $U(n)$ to $S p(2 n, \mathbb{R})$ by introducing scalar fields as in [64]. Although these remarks will be central in later arguments, their proofs closely resemble those in the case of complex fields, so we omit them. 


\section{Chapter 7}

\section{The Abelian Born-Infeld Action}

In this chapter, we introduce a generalization of the $U(1)$ Born-Infeld action with complex gauge fields and with a $U(n, n)$ self-duality group. In Section 7.1, we write the action using auxiliary fields, which greatly simplifies the check of duality invariance. We go on to eliminate the auxiliary fields in Section 7.2. We are then able to write down an explicit action without auxiliary fields by using a mathematical result about unilateral matrix equations, which is proven in Section 7.3.

\subsection{Born-Infeld with Auxiliary Fields}

In this section, we describe a $U(n, n)$ duality invariant nonlinear gauge theory with $n$ complex gauge fields [54]. The use of auxiliary fields in the Lagrangian is inspired by the work of $[55,56]$ and simplifies the check of duality invariance.

We begin with the following Lagrangian introduced in [54]

$$
L=\operatorname{Re} \operatorname{Tr}\left[i(\lambda-S) \chi-\frac{i}{2} \lambda \chi S_{2} \chi^{\dagger}+i \lambda \mathcal{N}\right]
$$

where $\mathcal{N}=\alpha-i \beta$. As mentioned in Chapter 6 , here we do not require $S$ to be symmetric. The auxiliary fields $\chi$ and $\lambda$ are $n$ dimensional complex matrices. If we could solve their equations of motion and use the solution in the Lagrangian (7.1) 
we would find a Lagrangian which depends only on $\alpha, \beta$ and $S$. Obtaining this Lagrangian is the main thrust of this chapter.

If we set $S=i$ in the above Lagrangian, the theory is only self-dual under the maximal compact subgroup $U(n) \times U(n)$, as discussed in Chapter 6. However, if we now reintroduce the scalar field as in (6.17), the new Lagrangian is the same as (7.1) only after field redefinitions of $\chi$ and $\lambda$. We can also add a kinetic term for the scalar field $S$. This term must be duality invariant since, as we will see shortly, the rest of the Lagrangian already satisfies the self-duality condition (6.11). For example we can add a nonlinear $\sigma$-model Lagrangian defined on the coset space $U(n, n) / U(n) \times U(n)$ with the metric given by

$$
\operatorname{Tr}\left[\left(S^{\dagger}-S\right)^{-1} d S^{\dagger}\left(S-S^{\dagger}\right)^{-1} d S\right]
$$

The metric (7.2) is Kähler since it is obtained from the Kähler potential

$$
K=\operatorname{Tr} \ln \left(S_{2}\right)
$$

This Kähler potential changes by a Kähler transformation under (6.20); this ensures that the metric is duality invariant.

It will be convenient to decompose the auxiliary fields into hermitian matrices, as we have already done for $S$,

$$
S=S_{1}+i S_{2}, \quad \lambda=\lambda_{1}+i \lambda_{2}, \quad \chi=\chi_{1}+i \chi_{2} .
$$

To prove the duality of (7.1) we first note that the last term in the Lagrangian can be written as

$$
\operatorname{Re} \operatorname{Tr}[i \lambda(\alpha-i \beta)]=\operatorname{Tr}\left(-\lambda_{2} \alpha+\lambda_{1} \beta\right)
$$


If the field $\lambda$ transforms by fractional transformation and the $\lambda_{i}$ 's and the gauge fields are real this is the $U(1)^{n}$ Maxwell action, with the gauge fields interacting with the scalar field $\lambda$, and this term by itself has the correct transformation properties under the duality group [3]. Similarly for hermitian $\alpha, \beta$ and $\lambda_{i}$ this term by itself satisfies equation (6.11). It follows that the rest of the Lagrangian must be duality invariant. The duality transformations of the scalar and auxiliary fields are

$$
\begin{aligned}
S^{\prime} & =(a S+b)(c S+d)^{-1}, \\
\lambda^{\prime} & =(a \lambda+b)(c \lambda+d)^{-1}, \\
\chi^{\prime} & =(c \lambda+d) \chi\left(c S^{\dagger}+d\right)^{\dagger} .
\end{aligned}
$$

To show the invariance of $\operatorname{Tr}[i(\lambda-S) \chi]$ it is convenient to rewrite (7.4) as

$$
S^{\prime}=\left(c S^{\dagger}+d\right)^{-\dagger}\left(a S^{\dagger}+b\right)^{\dagger}
$$

The proof of invariance of the remaining term which can be written as

$$
\operatorname{Re} \operatorname{Tr}\left[-\frac{i}{2} \lambda \chi S_{2} \chi^{\dagger}\right]=\operatorname{Tr}\left[\frac{1}{2} \lambda_{2} \chi S_{2} \chi^{\dagger}\right]
$$

is straightforward using the following transformations obtained from (7.4), (7.5) and (7.6)

$$
\begin{aligned}
S_{2}^{\prime} & =\left(c S^{\dagger}+d\right)^{-\dagger} S_{2}\left(c S^{\dagger}+d\right)^{-1}, \\
\lambda_{2}^{\prime} & =(c \lambda+d)^{-\dagger} \lambda_{2}(c \lambda+d)^{-1}, \\
\chi^{\prime \dagger} & =\left(c S^{\dagger}+d\right) \chi^{\dagger}(c \lambda+d)^{\dagger} .
\end{aligned}
$$


The Lagrangian has also a discrete parity symmetry which acts on the fields as

$$
\begin{aligned}
\alpha^{\prime} & =\bar{\alpha}, \\
\beta^{\prime} & =-\bar{\beta}, \\
S^{\prime} & =-\bar{S}, \\
\chi^{\prime} & =\bar{\chi}, \\
\lambda^{\prime} & =-\bar{\lambda} .
\end{aligned}
$$

Although the theory of duality invariance presented in the previous section guarantees that this theory is self-dual, one can also check directly that the equations of motion obtained by varying the auxiliary fields are preserved under duality rotations. These equations of motion are

$$
\begin{aligned}
& L_{\lambda} \equiv \frac{\partial L}{\partial \lambda^{T}}=i\left(\chi-\frac{1}{2} \chi S_{2} \chi^{\dagger}+\alpha-i \beta\right)=0, \\
& L_{\chi} \equiv \frac{\partial L}{\partial \chi^{T}}=i\left(\lambda-S-i S_{2} \chi^{\dagger} \lambda_{2}\right)=0
\end{aligned}
$$

and indeed these two equations form a multiplet under duality transformations. Using the explicit forms (7.9) and (7.10) one can check that

$$
\begin{aligned}
& \delta L_{\lambda}=(C \lambda+D) L_{\lambda}+L_{\lambda}\left(\lambda C+D^{\dagger}\right)+\chi L_{\chi} C, \\
& \delta L_{\chi}=-\left(S C+D^{\dagger}\right) L_{\chi}-L_{\chi}(C \lambda+D) .
\end{aligned}
$$

Alternatively, one can obtain these equations directly from (6.13).

\subsection{Elimination of the Auxiliary Fields}


In this section, we study the equation of motion (7.9) and attempt to solve for $\chi$. We then give the form that the Lagrangian assumes after the elimination of the auxiliary fields. This form is a generalization of the well-known Born-Infeld Lagrangian to more than one gauge field.

Using the equation of motion (7.9) in the Lagrangian (7.1), we obtain

$$
L=\operatorname{Re} \operatorname{Tr}[-i S \chi]=\operatorname{Tr}\left[S_{2} \chi_{1}+S_{1} \chi_{2}\right],
$$

where $\chi$ is now a function of $\alpha, \beta$ and $S_{2}$ that solves (7.9). For $n=1$, we have to solve a second order algebraic equation and we obtain

$$
\chi=\frac{1-\sqrt{1+2 S_{2} \alpha-S_{2}^{2} \beta^{2}}}{S_{2}}+i \beta .
$$

Apart from the fact that the gauge fields are complex, the result is the Born-Infeld Lagrangian

$$
L=1-\sqrt{1+2 S_{2} \alpha-S_{2}^{2} \beta^{2}}+S_{1} \beta .
$$

In fact, for $n=1$ we could have taken the gauge fields to be real even in the formulation with auxiliary fields as in [56], in which case the duality group becomes the $S p(2, \mathbb{R})$ subgroup of $U(1,1)$ obtained by requiring $a, b, c$ and $d$ to satisfy (6.16) and to be real.

We now study equation (7.9) for arbitrary $n$. First notice that (7.9) can be simplified with the following field redefinitions

$$
\begin{aligned}
& \hat{\chi}=R \chi R^{\dagger}, \\
& \widehat{\alpha}=R \alpha R^{\dagger}, \\
& \widehat{\beta}=R \beta R^{\dagger},
\end{aligned}
$$


where, as in (6.18), $R^{\dagger} R=S_{2}$. The equation of motion for $\chi$ is then equivalent to

$$
\widehat{\chi}-\frac{1}{2} \widehat{\chi} \widehat{\chi}^{\dagger}+\widehat{\alpha}-i \widehat{\beta}=0 .
$$

This is the same equation we would have obtained for $\chi$ if we would have set the scalar field $S=i$ initially, and thus broken the duality group to $U(n) \times U(n)$. For the rest of this section we will take this point of view, unless otherwise stated. Since we can always reintroduce the scalar field via the prescription given in Chapter 6 we choose to drop the tildes in the above equation. Then the equation of motion obtained by varying $\lambda$ gives the following equation for $\chi$

$$
\chi-\frac{1}{2} \chi \chi^{\dagger}+\alpha-i \beta=0,
$$

and after solving this equation the Lagrangian reduces to

$$
L=\operatorname{Re} \operatorname{Tr} \chi .
$$

Let $\chi=\chi_{1}+i \chi_{2}$ where $\chi_{1}$ and $\chi_{2}$ are hermitian. The anti-hermitian part of (7.15) implies $\chi_{2}=\beta$, thus $\chi^{\dagger}=\chi-2 i \beta$. This can be used to eliminate $\chi$ from (7.15) and obtain a quadratic equation for $\chi^{\dagger}$. It is convenient to define $Q=\frac{1}{2} \chi^{\dagger}$ which then satisfies

$$
Q=q+(p-q) Q+Q^{2},
$$

where

$$
p \equiv-\frac{1}{2}(\alpha-i \beta), \quad q \equiv-\frac{1}{2}(\alpha+i \beta) .
$$

The Lagrangian is then

$$
L=2 \operatorname{Re} \operatorname{Tr} Q .
$$


If the degree of the matrices is one, we can solve for $Q$ in the quadratic equation (7.16) and then (7.17) reduces to the Born-Infeld Lagrangian.

For matrices of higher degree, equation (7.16) can be solved perturbatively and by analyzing the first few terms in the expansion we conjectured in $[54,85]$ that the trace of $Q$ can be obtained as follows. First, find the perturbative solution of equation (7.16) assuming $p$ and $q$ commute. Then the trace of $Q$ is the trace of the symmetrized expansion

$$
\operatorname{Tr} Q=\frac{1}{2} \operatorname{Tr}\left[1+q-p-\mathcal{S} \sqrt{1-2(p+q)+(p-q)^{2}}\right],
$$

where the symmetrization operator $\mathcal{S}$ will be discussed in the next section. We have also found an explicit formula for the coefficients of the expansion of the trace of $Q$

$$
\operatorname{Tr} Q=\operatorname{Tr}\left[q+\sum_{r, s \geq 1}\left(\begin{array}{c}
r+s-2 \\
r-1
\end{array}\right)\left(\begin{array}{c}
r+s \\
r
\end{array}\right) \mathcal{S}\left(p^{r} q^{s}\right)\right] .
$$

In the next section, we will prove that for a unilateral matrix equation of order $N$, the perturbative solution is a sum of terms which are symmetrized in all the matrix coefficients as well as terms which are commutators. Since equation (7.16) is a unilateral matrix equation, the trace of $Q$ will be symmetrized in the matrix coefficients $q$ and $p-q$. This is equivalent to symmetrization in $q$ and $p$, and also equivalent to symmetrization in $\alpha$ and $\beta$. Because we know the solution $Q$ when $\alpha$ and $\beta$ commute, and now we know the ordering prescription, our conjecture (7.18) follows. In terms of $\alpha$ and $\beta$, the Lagrangian becomes

$$
L=\operatorname{Tr}\left[1-\mathcal{S} \sqrt{1+2 \widehat{\alpha}-\widehat{\beta}^{2}}+S_{1} \beta\right],
$$


where we have reintroduced the scalar field $S$ and enhanced the duality group to $U(n, n)$.

\subsection{Unilateral Matrix Equations}

In this section, we prove a theorem regarding certain solutions of unilateral matrix equations. These are $N^{\text {th }}$ order matrix equations for the variable $\phi$ with matrix coefficients $A_{i}$ which are all on one side, e.g. on the left

$$
\phi=A_{0}+A_{1} \phi+A_{2} \phi^{2}+\ldots+A_{N} \phi^{N} .
$$

The matrices are all square and of arbitrary degree. We may equally consider the $A_{i}$ 's as generators of an associative algebra, and $\phi$ an element of this algebra which satisfies the above equation. We will prove that the formal perturbative solution of (7.21) around zero is a sum of symmetrized polynomials in the $A_{i}$ and of terms which are commutators ${ }^{1}$. The same is true for all the positive powers of the solution.

By repeatedly inserting $\phi$ from the left hand side of (7.21) into the right hand side we obtain the perturbative expansion of $\phi$ as a sum

$$
\phi=\sum_{M} D_{M}
$$

where each $D_{M}$ is a product of the $A_{i}$ matrices. Any ordered product of these matrices will be referred to as a word. However not every word appears in the

\footnotetext{
${ }^{1}$ If the degree of the matrices is one the perturbative solution is convergent if $A_{0}$ and $A_{1}$ are sufficiently small.
} 
perturbative expansion of $\phi$. We reserve the letter $D$ for words that do appear ${ }^{2}$.

Next we obtain the condition that a word must satisfy in order to be in the expansion. First note that because of (7.21) any word $D_{M}$ can be written as the following product

$$
D_{M}=A_{s} D_{M_{1}} \ldots D_{M_{s}}
$$

for some value of $s$, where the $D_{M_{i}}$ 's are also words in the expansion. Conversely, if all the $D_{M_{i}}$ 's are words in the expansion, $D_{M}$ defined in equation (7.22) is also a word in the expansion. By iterating (7.22) we obtain the following equivalent statement: for every splitting of $D_{M}$ into two words $D_{M}=W_{1} W_{2}$ the second word can be written as a product of terms in the expansion of $\phi$

$$
D_{M}=W_{1} D_{N_{1}} \ldots D_{N_{k}} .
$$

It is convenient to assign to every matrix a dimension $d$ such that $d(\phi)=-1$. Using (7.21), the dimension of the matrix $A_{i}$ is given by $d\left(A_{i}\right)=i-1$ and $d\left(D_{M}\right)=$ -1 . Then we obtain the following intrinsic characterization of a word in the expansion of $\phi$. It is a word $D$ such that for every splitting into two words $D=W_{1} W_{2}$, where $W_{2}$ has at least one letter, we have

$$
d\left(W_{1}\right) \geq 0 \quad \text { and } \quad d(D)=-1 .
$$

\footnotetext{
${ }^{2}$ This notation originated from an earlier version of the proof where the perturbative expansion of $\phi$ was calculated diagrammatically and the diagrams were denoted by $D$. Although we will not use diagrams here, note that they are very useful in calculating the perturbative expansion of the solution.
} 
Note that (7.23) is a necessary and sufficient condition for a word to be in the expansion of $\phi$.

Suppose that $W$ is an arbitrary word such that $d(W)=-1$. Then, as we will show, there is a unique cyclic permutation $D$ of $W$ such that $D$ is a term in the expansion of $\phi$. Let us write $W=D_{N_{1}} D_{N_{2}} \ldots D_{N_{k}} W_{1}$, where $D_{N_{1}}$ is the shortest word starting from the first letter such that $d\left(D_{N_{1}}\right)=-1 . D_{N_{i}}$ is defined in the same way, except we start from the first letter after the word $D_{N_{i-1}}$. Finally $W_{1}$ is whatever is left over. We use the notation $D_{N_{i}}$ since they correspond to terms in the $\phi$ expansion. To see this, note that the total dimension of a word can increase or decrease when a letter is added on the right, but if it decreases it can only do so by one unit. This is when the letter added is $A_{0}$. Combining this with the fact that $D_{N_{i}}$ is the shortest word which satisfies $d\left(D_{N_{i}}\right)=-1$ then implies that if $D_{N_{i}}$ is a product of two words the dimension of the first word is greater than or equal to zero. This is just the condition (7.23). Then using the fact that $d\left(W_{1}\right)=k-1$ one can check that the cyclic permutation of $W$ defined as $D=W_{1} D_{N_{1}} \ldots D_{N_{k}}$ satisfies (7.23), thus it belongs to the expansion of $\phi$. Note that all the other cyclic permutations lead to words that are not in the expansion. Assuming the converse implies that two distinct terms in the expansion can be related by a cyclic permutation. But this is impossible: if we write $D=W_{1} W_{2}$, then $d\left(W_{1}\right) \geq 0$ and thus $d\left(W_{2}\right) \leq-1$, so that its cyclic permutation $W_{2} W_{1}$ does not satisfy (7.23). A similar argument can be used to show that all different cyclic permutations of a term in the expansion of $\phi$ lead to distinct words. 
Consider the trace of the sum of all distinct words of dimension $d=-1$ and of order $a_{i}$ in $A_{i}$. We can group together all words that are cyclic permutations of each other, and replace each group by a single word with coefficient $\sum_{i=0}^{N} a_{i}$. Using the result of the previous paragraph, we can choose this word to satisfy (7.23). Thus we have

$$
\operatorname{Tr}\left(\sum_{\text {order }\left\{a_{i}\right\}} D_{M}\right)=\left(\sum_{i=0}^{N} a_{i}\right)^{-1} \operatorname{Tr}\left(\sum_{\text {order }\left\{a_{i}\right\}} W\right),
$$

where the sum in the right hand side is over all distinct words of some fixed order $\left\{a_{i}\right\}$ and of dimension $d(W)=-1$.

We define the symmetrization operator $\mathcal{S}$ as a linear operator acting on monomials as

$$
\mathcal{S}\left(A_{0}^{a_{0}} A_{1}^{a_{1}} \ldots A_{N}^{a_{N}}\right)=\frac{a_{0} ! a_{1} ! \ldots a_{N} !}{\left(\sum_{i=0}^{N} a_{i}\right) !}\left(\sum_{\text {order }\left\{a_{i}\right\}} W\right),
$$

where the sum is over distinct words of fixed order $\left\{a_{i}\right\}$. Equivalently, a word can be symmetrized by averaging over all permutations of its letters. Not all permutations give distinct words and this accounts for the numerator on the right side of equation (7.25). The normalization of $\mathcal{S}$ is such that on commutative $A_{i}$ 's $\mathcal{S}$ acts as the identity.

Combining (7.24) and (7.25), we can obtain the solution for the trace of $\phi$ to all orders

$$
\operatorname{Tr} \phi=\sum_{\substack{\left\{a_{i}\right\} \\ \sum(i-1) a_{i}=-1}} \frac{\left(\sum_{i=0}^{N} a_{i}-1\right) !}{a_{0} ! a_{1} ! \ldots a_{N} !} \operatorname{Tr} \mathcal{S}\left(A_{0}^{a_{0}} A_{1}^{a_{1}} \ldots A_{N}^{a_{N}}\right)
$$

where the sum is over all sets $\left\{a_{i}\right\}$ restricted to words of dimension $d=-1$. More generally, if the $A_{i}$ 's are considered to be the generators of an associative algebra, 
we can replace the trace in (7.26) with the cyclic average operator which was defined in [85]. This is true since in the proof we only used the cyclic property of the trace which also holds for the cyclic average operator. Therefore, the solution $\phi$ can be written as a sum of symmetric polynomials and terms which are commutators. This is the statement we set out to prove. Notice that our derivation implies that the coefficients in (7.26) are all integers.

Using the same kind of arguments we used to derive equation (7.26), we can also prove that the trace of positive powers of $\phi$ is given by

$$
\operatorname{Tr} \phi^{r}=r \sum_{\substack{\left\{a_{i}\right\} \\ \sum(i-1) a_{i}=-r}} \frac{\left(\sum_{i=0}^{N} a_{i}-1\right) !}{a_{0} ! a_{1} ! \ldots a_{N} !} \operatorname{Tr} \mathcal{S}\left(A_{0}^{a_{0}} A_{1}^{a_{1}} \ldots A_{N}^{a_{N}}\right) .
$$

Furthermore we can write a generating function for (7.27)

$$
\operatorname{Tr} \log (1-\phi)=\left.\operatorname{Tr} \log \left(1-\sum_{i=0}^{N} A_{i}\right)\right|_{d<0} .
$$

On the right hand side of (7.28) one must expand the logarithm and restrict the sum to words of negative dimension. Since $d\left(\phi^{r}\right)=-r$ we can obtain (7.27) by extracting the dimension $d=-r$ terms from the right hand side of (7.28). Note that all the terms in the expansion of $\operatorname{Tr} \log \left(1-\sum_{i=0}^{N} A_{i}\right)$ are automatically symmetrized.

It is possible to give a simple proof of (7.28) without going through the combinatoric arguments above, which however give a construction of the solution and its powers themselves, not only their trace. First note that we can rewrite equation $(7.21)$ as

$$
1-\sum_{i=0}^{N} A_{i}=1-\phi-\sum_{k=1}^{N} A_{k}\left(1-\phi^{k}\right)
$$


The right hand side factorizes

$$
1-\sum_{i=0}^{N} A_{i}=\left(1-\sum_{k=1}^{N} \sum_{m=0}^{k-1} A_{k} \phi^{m}\right)(1-\phi) .
$$

Under the trace we can use the fundamental property of the logarithm, even for noncommutative objects, and obtain

$$
\operatorname{Tr} \log \left(1-\sum_{i=0}^{N} A_{i}\right)=\operatorname{Tr} \log \left(1-\sum_{k=1}^{N} \sum_{m=0}^{k-1} A_{k} \phi^{m}\right)+\operatorname{Tr} \log (1-\phi)
$$

Using $d\left(A_{k}\right)=k-1$ and $d(\phi)=-1$ we have $d\left(A_{k} \phi^{m}\right)=k-m-1$ and we see that all the words in the argument of the first logarithm on the right hand side have semi-positive dimension. Since all the words in the expansion of the second term have negative dimension we obtain (7.28).

If the coefficient $A_{N}$ is unity, we have the following identity for the symmetrization operator

$$
\left.\mathcal{S}\left(A_{0}^{a_{0}} A_{1}^{a_{1}} \ldots A_{N}^{a_{N}}\right)\right|_{A_{N}=1}=\mathcal{S}\left(A_{0}^{a_{0}} A_{1}^{a_{1}} \ldots A_{N-1}^{a_{N-1}}\right)
$$

This is obviously true up to normalization; the normalization can be checked in the commutative case.

The trace of the solution of (7.16) can now be obtained from (7.26) by taking $N=2$ and setting $A_{2}$ to unity. The restriction on the sum of (7.26) in this case reads $a_{0}-a_{2}=1$. The sum can then be rewritten

$$
\operatorname{Tr} \phi=\sum_{a_{0}=1}^{\infty} \sum_{a_{1}=0}^{\infty} \frac{\left(2 a_{0}+a_{1}-2\right) !}{a_{0} ! a_{1} !\left(a_{0}-1\right) !} \operatorname{Tr} \mathcal{S}\left(A_{0}^{a_{0}} A_{1}^{a_{1}}\right)
$$

Using $\phi=Q, A_{0}=q, A_{1}=p-q$, the combinatoric identity

$$
\left(\begin{array}{c}
a+b \\
c
\end{array}\right)=\sum_{m=\max (0, c-b)}^{\min (a, c)}\left(\begin{array}{c}
a \\
m
\end{array}\right)\left(\begin{array}{c}
b \\
c-m
\end{array}\right)
$$


and the resummation identities

$$
\begin{aligned}
\sum_{r \geq 1} \sum_{a=0}^{r} & =\sum_{a=0}^{\infty} \sum_{r=\max (a, 1)}^{\infty}, \\
\sum_{r=\max (a, 1)}^{\infty} \sum_{b=r-a+1}^{\infty} & =\sum_{b=\max (1,2-a)}^{\infty} \sum_{r=\max (a, 1)}^{a+b-1}
\end{aligned}
$$

one can show that (7.29) reduces to (7.19). 


\section{Chapter 8}

\section{Extensions of the Born-Infeld Action}

In this final chapter, we discuss extensions of our results from Chapter 7 . In Section 8.1, we argue that by replacing the complex gauge fields of Chapter 7 with real ones, we obtain a Born-Infeld theory with the maximal duality group. We discuss supersymmetric extensions in Section 8.2. And finally, in Section 8.3, we find that similar Born-Infeld theories can be defined in flat spacetime of any even dimension.

\subsection{Real Field Strengths}

We now show that our results imply the existence of a Born-Infeld theory with $n$ real field strengths which is duality invariant under the maximal duality group $S p(2 n, \mathbb{R})$. We first study the case without scalar fields, i.e. $S_{1}=0$ and $S_{2}=R=1$. Consider a Lagrangian $\mathcal{L}=\mathcal{L}(\alpha, \beta)$ which describes a self-dual theory with complex gauge fields. We will assume that the Lagrangian is a sum of traces (or of products of traces) of monomials in $\alpha$ and $\beta$. It follows that this Lagrangian satisfies the self-duality equations (6.30). This remains true in the special case that $\alpha$ and $\beta$ are real. That is $\mathcal{L}=\mathcal{L}(\alpha, \beta)$ satisfies the selfduality equation (6.30) with $\alpha=\alpha^{T}=\bar{\alpha}$ and $\beta=\beta^{T}=\bar{\beta}$. We now recall that 
equation (6.30) is also the self-duality condition for Lagrangians with real gauge fields provided that $\alpha$ and $\beta$ are defined in the following way

$$
\alpha^{a b}=\frac{1}{4} F_{R}^{a} F_{R}^{b}, \quad \beta^{a b}=\frac{1}{4} \widetilde{F}_{R}^{a} F_{R}^{b},
$$

where $F_{R}^{a}$ denotes a real field strength. This implies that the theory described by the Lagrangian $\mathcal{L}_{R}=\mathcal{L}\left(\alpha\left(F_{R}^{a}\right), \beta\left(F_{R}^{a}\right)\right)$ is self-dual with duality group $U(n)$, the maximal compact subgroup of $S p(2 n, \mathbb{R})$. The duality group can be extended to the full noncompact $S p(2 n, \mathbb{R})$, the maximal duality group of $n$ real field strengths [3], by introducing the scalar fields $S$ via the prescription (6.17) which also applies to the real case provided $S$ is symmetric [64].

In our case the Lagrangian $L=\operatorname{Tr}\left[\chi_{1}(\widehat{\alpha}, \widehat{\beta})+S_{1} \beta\right.$, $]$, where $\chi(\widehat{\alpha}, \widehat{\beta})$ is the solution of (7.15), defines a duality invariant theory because it is obtained from the Lagrangian with auxiliary fields (7.1) that is explicitly self-dual. Therefore $L_{R}=\operatorname{Tr}\left[\chi_{1}(\widehat{\alpha}, \widehat{\beta})+S_{1} \beta,\right]$, with the field strengths taken real is also self-dual. Using (7.20) we obtain an explicit formula for the Born-Infeld Lagrangian with real gauge fields describing an $S p(2 n, \mathbb{R})$ duality invariant theory

$$
L_{R}=\operatorname{Tr}\left[1-\mathcal{S} \sqrt{1+2 \widehat{\alpha}-\widehat{\beta}^{2}}+S_{1} \beta\right]
$$

\subsection{Supersymmetric Theory}

In this section, we briefly discuss supersymmetric versions of some of the Lagrangians introduced. First, we discuss the supersymmetric form of the La-

grangian (7.1). Consider the superfields $V^{a}=\frac{1}{\sqrt{2}}\left(V_{1}^{a}+i V_{2}^{a}\right)$ and $\check{V}^{a}=\frac{1}{\sqrt{2}}\left(V_{1}^{a}-\right.$ 
$\left.i V_{2}^{a}\right)$ where $V_{1}^{a}$ and $V_{2}^{a}$ are real vector superfields, and define

$$
W_{\alpha}^{a}=-\frac{1}{4} \bar{D}^{2} D_{\alpha} V^{a}, \quad \check{W}_{\alpha}^{a}=-\frac{1}{4} \bar{D}^{2} D_{\alpha} \check{V}^{a}
$$

Both $W^{a}$ and $\check{W}^{a}$ are chiral superfields and can be used to construct a matrix of chiral superfields

$$
\mathcal{M}^{a b} \equiv W^{a} \check{W}^{b}
$$

The supersymmetric version of the Lagrangian (7.1) is then given by

$$
L=\operatorname{Re} \int d^{2} \theta\left[\operatorname{Tr}\left(i(\lambda-S) \chi-\frac{i}{2} \lambda \bar{D}^{2}\left(\chi S_{2} \chi^{\dagger}\right)-i \lambda \mathcal{M}\right)\right]
$$

where $S, \lambda$ and $\chi$ denote chiral superfields with the same symmetry properties as their corresponding bosonic fields. While the bosonic fields $S$ and $\lambda$ appearing in (7.1) are the lowest component of the superfields denoted by the same letter, the field $\chi$ in the action (7.1) is the highest component of the superfield $\chi$. A supersymmetric kinetic term for the scalar field $S$ can be written using the Kähler potential (7.3) as described in [86].

Just as in the bosonic Born-Infeld, one would like to eliminate the auxiliary fields. However we have not been able to do this exactly except for $n=1$, and unlike the bosonic case we do not even have a conjectured form of the Lagrangian without auxiliary fields. For $n=1$ just as in the bosonic case the theory with auxiliary fields also admits both a real and a complex version, i.e. we can also consider a Lagrangian with a single real superfield. Then we can integrate out the auxiliary superfields and obtain the supersymmetric version of (7.20)

$$
L=\int d^{4} \theta \frac{S_{2}^{2} W^{2} \bar{W}^{2}}{1-A+\sqrt{1-2 A+B^{2}}}+\operatorname{Re}\left[\int d^{2} \theta\left(-\frac{i}{2} S W^{2}\right)\right]
$$


where

$$
A=\frac{1}{4}\left(D^{2}\left(S_{2} W^{2}\right)+\bar{D}^{2}\left(S_{2} \bar{W}^{2}\right)\right), \quad B=\frac{1}{4}\left(D^{2}\left(S_{2} W^{2}\right)-\bar{D}^{2}\left(S_{2} \bar{W}^{2}\right)\right) .
$$

If we only want a $U(1)$ duality invariance we can set $S=i$ and then the action (8.3) reduces to the supersymmetric Born-Infeld action described in [57, 58, 59].

In the case of weak fields, the first term of (8.3) can be neglected and the Lagrangian is quadratic in the field strengths. Under these conditions, the combined requirements of supersymmetry and self duality can be used [87] to constrain the form of the weak coupling limit of the effective Lagrangian from string theory.

\subsection{Extension to Arbitrary Even Dimensions}

In a space-time of arbitrary even dimension, $D=2 p$ we define the matrices

$$
\alpha^{a b}=\frac{1}{p !} F_{\mu_{1} \ldots \mu_{p}}^{a} \bar{F}^{b \mu_{1} \ldots \mu_{p}}, \quad \beta^{a b}=\frac{1}{p !} \tilde{F}_{\mu_{1} \ldots \mu_{p}}^{a} \bar{F}^{b \mu_{1} \ldots \mu_{p}}
$$

where $\tilde{F}_{\mu_{1} \ldots \mu_{p}}^{a}=1 / p ! \varepsilon_{\mu_{1} \ldots \mu_{p} \nu_{1} \ldots \nu_{p}} F^{a \nu_{1} \ldots \nu_{p}}$ is the Hodge dual of $F^{a}$. The dual field strength is given by

$$
\tilde{G}_{\mu_{1} \ldots \mu_{p}}^{a} \equiv p ! \frac{\partial L}{\partial \bar{F}^{a \mu_{1} \ldots \mu_{p}}}, \quad \widetilde{\bar{G}}_{\mu_{1} \ldots \mu_{p}}^{a} \equiv p ! \frac{\partial L}{\partial F^{a \mu_{1} \ldots \mu_{p}}}
$$

Since $\widetilde{\widetilde{F}}=(-1)^{p+1} F$ and $\tilde{F} G=(-1)^{p} F \tilde{G}$, for all even dimensions the matrix $\alpha$ is hermitian, while $\beta$ is hermitian if $D=4 \nu$ and anti-hermitian if $D=4 \nu+2$. It is also convenient to define

$$
\mathcal{N}= \begin{cases}\alpha-i \beta, & \text { if } D=4 \nu \\ \alpha+\beta, & \text { if } D=4 \nu+2\end{cases}
$$


With these definitions the Lagrangian (7.1) gives a $U(n, n)$ duality invariant theory in arbitrary even dimensions.

However, if the dimension of space-time is $D=4 \nu+2$, where $\nu$ is integer it is convenient to make the following field redefinitions

$$
\Lambda=i \lambda, \quad \mathcal{S}=i S
$$

The new fields have the decomposition

$$
\Lambda=-\Lambda_{1}+\Lambda_{2}, \mathcal{S}=-\mathcal{S}_{1}+\mathcal{S}_{2}
$$

where $\Lambda_{1}$ and $\mathcal{S}_{1}$ are hermitian and $\Lambda_{2}$ and $\mathcal{S}_{2}$ are anti-hermitian. The minus sign was introduced so that we have

$$
\mathcal{S}_{1}=S_{2}
$$

Then $\mathcal{S}_{1}$ is positive definite and we can write $\mathcal{S}_{1}=R^{\dagger} R$ with $R$ an arbitrary nonsingular $n$-dimensional matrix.

We also perform a similarity transformation on the $U(n, n)$ duality group, such that the transformation properties of the new fields simplify. Let us define two $2 n$-dimensional matrices with the block form

$$
\mathbb{K}=\left(\begin{array}{rr}
0 & 1 \\
-1 & 0
\end{array}\right), \quad \mathbb{H}=\left(\begin{array}{ll}
0 & 1 \\
1 & 0
\end{array}\right)
$$

and let the matrices $T$ and $M$ have the block decomposition

$$
M=\left(\begin{array}{ll}
a & b \\
c & d
\end{array}\right), T=\left(\begin{array}{ll}
\underline{a} & \underline{b} \\
\underline{c} & \underline{d}
\end{array}\right) .
$$


Then one can define the $U(n, n)$ group as the group of matrices satisfying either one of the two relations

$$
M \mathbb{K} M^{\dagger}=\mathbb{K}, \quad T \mathbb{H} T^{\dagger}=\mathbb{H} .
$$

The two definitions are related by a unitary transformation $M=\mathcal{U}^{-1} T \mathcal{U}$ where

$$
\mathcal{U}=\left(\begin{array}{cc}
e^{i \pi / 4} & 0 \\
0 & e^{-i \pi / 4}
\end{array}\right) .
$$

The $n$-dimensional matrices $\underline{a}, \underline{b}, \underline{c}$ and $\underline{d}$ satisfy

$$
\underline{a}^{\dagger} \underline{d}+\underline{c}^{\dagger} \underline{b}=1, \quad \underline{c}^{\dagger} \underline{a}+\underline{a}^{\dagger} \underline{c}=0, \quad \underline{b}^{\dagger} \underline{d}+\underline{d}^{\dagger} \underline{b}=0 .
$$

The action of $U(n, n)$ on the scalar fields is given by

$$
\begin{aligned}
\mathcal{S}^{\prime} & =(\underline{a} \mathcal{S}+\underline{b})(\underline{c} \mathcal{S}+\underline{d})^{-1}, \\
\Lambda^{\prime} & =(\underline{a} \Lambda+\underline{b})(\underline{c} \Lambda+\underline{d})^{-1}, \\
\chi^{\prime} & =(\underline{c} \Lambda+\underline{d}) \chi\left(-\underline{c} \mathcal{S}^{\dagger}+\underline{d}\right)^{\dagger} .
\end{aligned}
$$

Note that the positivity of $\mathcal{S}_{1}$ is compatible with the above transformation law of $\mathcal{S}$.

The Lagrangian, written in terms of the redefined fields, takes the form

$$
L=\operatorname{Re}\left[\operatorname{Tr}\left((\Lambda-\mathcal{S}) \chi-\frac{1}{2} \Lambda \chi \mathcal{S}_{1} \chi^{\dagger}+\Lambda \mathcal{N}\right)\right] .
$$

Our conjecture regarding the Lagrangian without auxiliary fields is independent of the dimension of space-time and if it holds we can eliminate the auxiliary fields to obtain the Lagrangian

$$
L=\operatorname{Tr}\left[1-\mathcal{S} \sqrt{1+2 \widehat{\alpha}+\widehat{\beta}^{2}}+\mathcal{S}_{2} \beta\right],
$$


where

$$
\begin{aligned}
& \widehat{\alpha}=R \alpha R^{\dagger}, \\
& \widehat{\beta}=R \beta R^{\dagger} .
\end{aligned}
$$

Note also that $\mathcal{S}_{2}$ appears in the last term of the Lagrangian (8.11), and this is consistent with $\mathcal{S}_{2}$ and $\beta$ being anti-hermitian in space-times of odd half dimension. Also, there is a change of sign in front of the $\hat{\beta}^{2}$ term under the square root in (8.11) due to the change in the definition of $\mathcal{N}$.

If the half-dimension of space-time is odd it is consistent to take all the fields to be real in either the Lagrangian with auxiliary fields (8.10), or in the Lagrangian (8.11) where the auxiliary fields have been eliminated. Then we obtain a theory invariant under an $O(n, n)$ duality group. It was shown in $[60,64]$ that the maximal connected duality group for a theory of dimension $D=4 \nu+2$ with $n$ antisymmetric tensors is $S O(n, n)$. In the analysis of $[60,64]$ only infinitesimal duality transformations were considered, and from these one can only show duality under the connected component of the group. In $[62,63]$ the group $O(n, n)$ was considered. Note that, as discussed in Appendix C, $O(n, n)$ has four disjoint components embedded in $U(n, n)$ which is a connected group. Finally, one can also obtain a theory invariant under the $O(n) \times O(n)$ maximal compact subgroup of $O(n, n)$ by setting $\mathcal{S}=-1$ in the Lagrangian (8.11). 


\section{Appendix A}

\section{Chiral Spinor Representations of $S O(d, d \mid \mathbf{Z})$}

In the first part of this appendix it will be shown that the Weyl spinor representations of $S O(d, d \mid \mathbf{Z})$ are integral, i.e. have matrix elements which are integers.

In the final part, it is proven that for $d=3$ the Weyl spinor representation is in fact isomorphic to $S L(4, \mathbf{Z})$.

The gamma matrices obeying (2.47), where the metric has the form (2.45), are already, up to normalization, the standard creation and annihilation operators used to generate the Fock space for Dirac spinors in the Weyl basis. These are defined as

$$
a_{i}^{\dagger}=\gamma_{i} / \sqrt{2}, \quad a_{i}=\gamma_{d+i} / \sqrt{2}
$$

and satisfy the canonical anti-commutation relations

$$
\left\{a_{i}, a_{j}^{\dagger}\right\}=\delta_{i j}, \quad\left\{a_{i}, a_{j}\right\}=\left\{a_{i}^{\dagger}, a_{j}^{\dagger}\right\}=0, \quad i, j=1, \ldots, d .
$$

As usual, the Dirac spinor and vector representations are related through formula (2.46) in the main text

$$
\mathcal{S}^{-1} \gamma_{s} \mathcal{S}=\Lambda_{s}^{p} \gamma_{p}
$$

To prove that the Weyl spinor representations are integral we will use a theorem presented in [38] where it was shown that the whole group $S O(d, d \mid \mathbf{Z})$ is generated 
by a special subset of group elements. An explicit construction of the Weyl spinor representation matrices corresponding to the group elements in that subset is given and shown to be integral. The subset contains three types of elements. The first type are generators of the form

$$
\left(\begin{array}{cc}
I_{d} & n \\
0 & I_{d}
\end{array}\right), \quad n^{T}=-n .
$$

The second type of generators forming a $S L(d, \mathbf{Z}) \times \mathbf{Z}_{2}$ subgroup have the form

$$
\left(\begin{array}{cc}
R & 0 \\
0 & R^{T^{-1}}
\end{array}\right), \quad \operatorname{det} R= \pm 1 .
$$

These are the T-duality generators corresponding to a change of basis of the of the compactification lattice.

The final generator is given by

$$
\left(\begin{array}{llllll}
0 & & & 1 & & \\
& 0 & & & 1 & \\
& & I_{d-2} & & & 0_{d-2} \\
1 & & & 0 & & \\
& 1 & & & 0 & \\
& & & & & \\
& & 0_{d-2} & & & I_{d-2}
\end{array}\right) .
$$

It corresponds to T-duality along the $x^{1}$ and $x^{2}$ coordinates. The full duality group is in fact $O(d, d \mid \mathbf{Z})$ but here we will only consider its restriction to $S O(d, d \mid \mathbf{Z})$ which is the subgroup that does not exchange Type IIA and IIB. The full T-duality group is then obtained by adding to the above list one more generator corresponding to T-duality in a single direction.

Using (2.46) one can check that the Dirac spinor representation corresponding 
to the first type of generator (A.1) is

$$
\exp \left(\frac{1}{2} n_{i j} a_{i} a_{j}\right)
$$

This has a finite expansion and is manifestly integer valued in the standard Fock space basis obtained by acting with the creation operators on a vacuum state.

One can prove that the full $S L(d, \mathbf{Z})$ group is generated by its $S L(2, \mathbf{Z})_{i j}$ subgroups acting on the $x^{i}$ and $x^{j}$ coordinates. One can use this to find the spinor representation matrices corresponding to generators of the second type (A.2). Since each $S L(2, \mathbf{Z})_{i j}$ is generated by its $T_{i j}$ and $S_{i j}$ transformations, which in the $(i j)$ subspace where $i<j$ have the form

$$
\left(\begin{array}{ll}
1 & 1 \\
0 & 1
\end{array}\right), \quad\left(\begin{array}{cc}
0 & -1 \\
1 & 0
\end{array}\right),
$$

it is enough to find the spinor matrices for these generators. The spinor representation of $T_{i j}$ is given by

$$
\exp \left(a_{i} a_{j}^{\dagger}\right)
$$

The exponential (A.5) has a finite expansion and its matrix elements are integer valued. Similarly the spinor representation of $S_{i j}$ is given by

$$
\exp \left(\frac{\pi}{2}\left(a_{j}^{\dagger} a_{i}-a_{i}^{\dagger} a_{j}\right)\right)
$$

Let us define $A=a_{j}^{\dagger} a_{i}-a_{i}^{\dagger} a_{j}$ for fixed values of $i$ and $j$. In terms of number operators $N_{i}=a_{i}^{\dagger} a_{i}$ we have $A^{2}=-N_{i}-N_{j}+2 N_{i} N_{j}$. Since $N_{i}$ can be either zero or one, $A^{2}$ is zero or minus one. The Fock space can be split into a direct sum of two subspaces, defined by the eigenvalues of $A^{2}$. On the subspace defined by 
$A^{2}=0$, one also has $A=0$ and thus the spinor representation (A.6) reduces to the identity. On the subspace defined by $A^{2}=-1$, the exponential can be written as $\cos (\pi / 2)+A \sin (\pi / 2)=A$. On both subspaces, the representation matrix of the transformation is integer valued. A formula for the spinor representation of the $S_{i j}$ generators which is valid on both subspaces is given by, $1+A+A^{2}$. The second type of generator (A.2) also contains elements with $\operatorname{det} R=-1$. A spinor transformation corresponding to such a generator is given by

$$
1-2 a_{1}^{\dagger} a_{1}
$$

Finally, the generator (A.3) has the spinor representation

$$
\exp \left(\frac{\pi}{2}\left(a_{1}-a_{1}^{\dagger}\right)\left(a_{2}-a_{2}^{\dagger}\right)\right)
$$

It has a finite expansion given by $\left(a_{1}-a_{1}^{\dagger}\right)\left(a_{2}-a_{2}^{\dagger}\right)$, which can be obtained using $\left(\left(a_{1}-a_{1}^{\dagger}\right)\left(a_{2}-a_{2}^{\dagger}\right)\right)^{2}=-1$, and in this form it is manifestly integral.

Since the Fock space basis used splits into two subsets of definite chirality, it follows that the Weyl spinor representations of $S O(d, d \mid \mathbf{Z})$ are also integral.

In the remainder of the appendix it is shown that the Weyl spinor representation of $S O(3,3 \mid \mathbf{Z})$ is isomorphic to $S L(4, \mathbf{Z})$. First note that for the Lie algebra corresponding to the continuous Lie groups we have the equivalence so $(3,3 \mid \mathbf{R}) \cong \operatorname{sl}(4, \mathbf{R})$. The spinor representation of the first group is isomorphic to the fundamental of the second. Since in the first part of the appendix it was shown that the spinor representations are integral it is reasonable to expect that they form a subgroup of $S L(4, \mathbf{Z})$. In fact we will show that they are isomorphic 
to the whole $S L(4, \mathbf{Z})$ group.

One can represent the Weyl spinor state $n|0\rangle+\frac{1}{2} M^{i j} a_{i}^{\dagger} a_{j}^{\dagger}|0\rangle$ as the column

$$
\left(\begin{array}{c}
n \\
M^{23} \\
M^{31} \\
M^{12}
\end{array}\right)
$$

Using operators of the form (A.5) and (A.6) one generates an $S L(3, \mathbf{Z})$ subgroup of the form

$$
\left(\begin{array}{ll}
1 & 0 \\
0 & R
\end{array}\right)
$$

where $R$ is the same matrix appearing in (A.2). We will now show that the Weyl spinor representation also contains $S L(2, \mathbf{Z})_{1 i}$ subgroups which act on the first and the $i+1$ entries of the column spinor (A.9). These subgroups together with (A.10) generate the entire $S L(4, \mathbf{Z})$ group. The T-duality generator (A.8), denoted below $T_{12}$, has the Weyl spinor representation

$$
T_{12}=\left(\begin{array}{cccc}
0 & 0 & 0 & -1 \\
0 & 0 & -1 & 0 \\
0 & 1 & 0 & 0 \\
1 & 0 & 0 & 0
\end{array}\right) .
$$

Let us also consider a transformation $G$ given by

$$
G=\left(\begin{array}{cccc}
1 & 0 & 0 & 0 \\
0 & 1 & 0 & 0 \\
0 & 0 & a & b \\
0 & 0 & c & d
\end{array}\right), \quad a d-b c=1,
$$

which is an element of an $S L(2, \mathbf{Z})$ subgroup of elements of the form (A.10). By 
conjugating $G$ with the $T_{12}$ generator

$$
T_{12}^{-1} G T_{12}=\left(\begin{array}{cccc}
d & c & 0 & 0 \\
b & a & 0 & 0 \\
0 & 0 & 1 & 0 \\
0 & 0 & 0 & 1
\end{array}\right),
$$

one finds an $S L(2, \mathbf{Z})_{12}$ transformation acting on the first and second entries. All the other $S L(2, \mathbf{Z})_{1 i}$ subgroups can be obtained by conjugating (A.11) with elements of the form (A.10). Thus we have found Weyl spinor representations generating the entire $S L(4, \mathbf{Z})$ group. In fact the representation is isomorphic to $S L(4, \mathbf{Z})$ since all the spinor representation matrices (A.4), (A.5), (A.6), (A.7) and (A.8) are integral and have unit determinant. 


\section{Appendix B}

\section{Duality Invariants at Small Compactification}

\section{Volume}

This Appendix contains some mathematical results regarding the spinor representation of the T-duality group and duality invariant quantities in the small volume limit. The group $S O(d, d)$ is the group of $2 d$-dimensional matrices $\Lambda$ satisfying $\Lambda J \Lambda^{T}=J$ where $J$ is a matrix with the block form

$$
J=\left(\begin{array}{ll}
0 & 1 \\
1 & 0
\end{array}\right)
$$

It will be useful to know how to calculate the Weyl spinor representation matrix of an $S O(3,3)$ group element $\Lambda$ with the block form

$$
\Lambda=\left(\begin{array}{ll}
\mathcal{A} & \mathcal{B} \\
\mathcal{C} & \mathcal{D}
\end{array}\right)
$$

First note that if $\mathcal{A}$ is invertible $\Lambda$ has a block Gauss decomposition

$$
\Lambda=\left(\begin{array}{cc}
1 & 0 \\
\mathcal{C A}^{-1} & 1
\end{array}\right)\left(\begin{array}{cc}
\mathcal{A} & 0 \\
0 & \mathcal{A}^{-T}
\end{array}\right)\left(\begin{array}{cc}
1 & \mathcal{A}^{-1} \mathcal{B} \\
0 & 1
\end{array}\right)
$$

where one can show using the group relations that $\mathcal{C} \mathcal{A}^{-1}$ and $\mathcal{A}^{-1} \mathcal{B}$ are antisymmetric. This decomposition is in fact true for generic $S O(d, d)$ matrices. For $d=3$ one can give the explicit spinor representation matrices for each factor in (B) thus 
obtaining the spinor representation of a generic $S O(3,3)$ matrix $\Lambda$

$$
S=\left(\begin{array}{cc}
1 & 0 \\
*\left(\mathcal{C} \mathcal{A}^{-1}\right) & 1
\end{array}\right)\left(\begin{array}{cc}
\operatorname{det} \mathcal{A}^{1 / 2} & 0 \\
0 & \operatorname{det} \mathcal{A}^{-1 / 2} \mathcal{A}
\end{array}\right)\left(\begin{array}{cc}
1 & *\left(\mathcal{A}^{-1} \mathcal{B}\right)^{T} \\
0 & 1
\end{array}\right)
$$

The star denotes the duality operator. When acting on antisymmetric 3dimensional square matrices it gives the dual column matrix.

Invariants can be constructed using two column matrices transforming in the vector representation of $S O(d, d \mid \mathbf{Z})$ and the symmetric $S O(d, d \mid \mathbf{Z})$ matrix $\mathcal{M}$

$$
\left(r^{T}, s^{T}\right) \mathcal{M}\left(\begin{array}{l}
u \\
v
\end{array}\right)
$$

In the limit when $G$ goes to zero, using the block Gauss decomposition of $\mathcal{M}$

$$
\mathcal{M}=\left(\begin{array}{ll}
1 & 0 \\
B & 1
\end{array}\right)\left(\begin{array}{cc}
G^{-1} & 0 \\
0 & G
\end{array}\right)\left(\begin{array}{cc}
1 & -B \\
0 & 1
\end{array}\right)
$$

and after identifying $B$ with $\Theta$ one obtains the following invariant ${ }^{1}$

$$
(r-\Theta s)^{T} G^{-1}(u-\Theta v)
$$

Using the transformation of $G$ under the duality group (1.5) one can write the transformation of $u-\Theta v$

$$
(\bar{u}-\bar{\Theta} \bar{v})=(\mathcal{C} \Theta+\mathcal{D})^{-T}(u-\Theta v) .
$$

The spinor representation matrix of $\mathcal{M}$ can be calculated using (B.2)

$$
S(\mathcal{M})=\left(\begin{array}{ll}
1 & 0 \\
b & 1
\end{array}\right)\left(\begin{array}{cc}
\operatorname{det} G^{-1 / 2} & 0 \\
0 & \operatorname{det} G^{1 / 2} G^{-1}
\end{array}\right)\left(\begin{array}{cc}
1 & b^{T} \\
0 & 1
\end{array}\right) .
$$

\footnotetext{
${ }^{1}$ To obtain a finite result, one should insert appropriate factors of $\alpha^{\prime}$ in (B.3) and also take $\alpha^{\prime}$ to zero as discussed in the Introduction.
} 
Then one can also form the invariants $\eta^{T} S(\mathcal{M}) \chi$ using two chiral spinors

$$
\chi=\left(\begin{array}{l}
\chi_{0} \\
\chi_{1} \\
\chi_{2} \\
\chi_{3}
\end{array}\right), \quad \eta=\left(\begin{array}{l}
\eta_{0} \\
\eta_{1} \\
\eta_{2} \\
\eta_{3}
\end{array}\right) .
$$

In the limit of vanishing $G$, the invariant becomes

$$
\left(\eta_{0}+\frac{1}{2} \varepsilon^{i j k} \Theta_{i j} \eta_{k}\right) \sqrt{\operatorname{det} G^{-1}}\left(\chi_{0}+\frac{1}{2} \varepsilon^{i j k} \Theta_{i j} \chi_{k}\right)
$$

From (B.5) one obtains the following transformation law

$$
\bar{\chi}_{0}+\frac{1}{2} \varepsilon^{i j k} \bar{\Theta}_{i j} \bar{\chi}_{k}=|\operatorname{det}(\mathcal{C} \Theta+\mathcal{D})|^{-1 / 2}\left(\chi_{0}+\frac{1}{2} \varepsilon^{i j k} \Theta_{i j} \chi_{k}\right) .
$$

One can also check the relations (B.4) and (B.6) directly using the transformations (1.4) of $\Theta$.

Given two chiral spinors $\chi$ and $\eta$ first write them as Dirac spinors

$$
\chi_{D}=\left(\begin{array}{l}
\chi \\
0
\end{array}\right), \quad \eta_{D}=\left(\begin{array}{l}
\eta \\
0
\end{array}\right) \text {. }
$$

Then using the same definition for $a^{i}$ and $a_{i}^{\dagger}$ as in Appendix A one can form the $S O(3,3 \mid \mathbf{Z})$ vector

$$
\left(\begin{array}{c}
u_{i} \\
v^{i}
\end{array}\right)=\bar{\eta}_{D}\left(\begin{array}{c}
a_{i}^{\dagger} \\
a^{i}
\end{array}\right) \chi_{D} .
$$

where $\bar{\eta}_{D}=\eta^{\dagger} T$. Here $T$ is a matrix acting on Dirac spinors and plays the same role as $\gamma_{0}$ when one forms barred spinors in Minkowski space. It is given by

$$
T=\left(a_{1}^{\dagger}+a^{1}\right)\left(a_{2}^{\dagger}+a^{2}\right)\left(a_{3}^{\dagger}+a^{3}\right) .
$$

Writing out all the spinor components in (B.7) one has

$$
\left(\begin{array}{c}
u_{i} \\
v^{i}
\end{array}\right)=\left(\begin{array}{c}
\eta_{i} \chi_{0}-\eta_{0} \chi_{i} \\
\varepsilon^{i j k} \eta_{j} \chi_{k}
\end{array}\right)
$$


Then $u-\Theta v$ transforms as in (B.4) under the duality group. Such an expression, involving two chiral spinors and $\Theta$, is used in the main text. 


\section{Appendix $\mathrm{C}$}

\section{Parametrizations of Coset Spaces $G / H$}

In this appendix we show that the field $S$ provides a global parametrization of the coset space $G / H$ where $G$ is $U(n, n), S p(2 n, \mathbb{R})$ or $O(n, n)$ and $H$ is the maximal compact subgroup of $G$. We will concentrate on $U(n, n)$ but the same argument applies for the other groups.

Cosets are equivalence classes of group elements $g$ of $G$ under right multiplication with arbitrary elements $h$ of $H$

$$
g \sim g h
$$

We denote the coset containing $g$ by $g H$. The maximal compact subgroup of $G$ is defined as

$$
H \equiv\left\{h \in G \mid h h^{\dagger}=h^{\dagger} h=1\right\}
$$

It is the intersection of $U(n, n)$ with $U(2 n)$ i.e. $U(n) \times U(n)$.

Next consider the map $\phi: G / H \rightarrow C$ defined by

$$
\phi(g H)=g g^{\dagger}
$$

where

$$
C=\left\{s \in G \mid s^{\dagger}=s, s \text { positive definite }\right\}
$$


is the subset of hermitian positive definite group elements of $G$. This map is well defined since for any two elements $g$ and $g^{\prime}$ in the same coset, $g^{\prime}=g h$ and $g^{\prime} g^{\prime \dagger}=g h h^{\dagger} g^{\dagger}=g g^{\dagger}$. Furthermore this map is one to one. We show first that the map is surjective. Let $s$ be an arbitrary hermitian positive definite element of $G$.

Then

$$
s=\left(\begin{array}{ll}
a & b \\
c & d
\end{array}\right)=\left(\begin{array}{cc}
1 & b d^{-1} \\
0 & 1
\end{array}\right)\left(\begin{array}{cc}
d^{-\dagger} & 0 \\
0 & d
\end{array}\right)\left(\begin{array}{cc}
1 & 0 \\
d^{-1} c & 1
\end{array}\right) .
$$

The last equality in (C.1) can be checked using the group relations (6.16). The decomposition exists whenever $d$ is invertible, but since $s$ is positive definite and $d$ is the restriction of $s$ on an $n$-dimensional subspace $d$ is also positive definite. Note also that $d^{\dagger}=d$ and $\left(b d^{-1}\right)^{\dagger}=b d^{-1}=d^{-1} c$. Then $g$ defined as

$$
g=\left(\begin{array}{cc}
1 & b d^{-1} \\
0 & 1
\end{array}\right)\left(\begin{array}{cc}
d^{-1 / 2} & \\
0 & d^{1 / 2}
\end{array}\right)
$$

satisfies $s=g g^{\dagger}$, thus the map $\phi$ is surjective. To show that the map is also injective note that $g g^{\dagger}=g^{\prime} g^{\prime \dagger}$ is equivalent to $g^{\prime-1} g\left(g^{\prime-1} g\right)^{\dagger}=1$. Then $h=g^{\prime-1} g$ is an element of $G$ satisfying $h h^{\dagger}=1$, that is it belongs to the maximal compact subgroup $\mathrm{H}$ and we have $g=g^{\prime} h$ so $g$ and $g^{\prime}$ belong to the same coset.

If we define $S_{2}=d^{-1}$ and $S_{1}=b d^{-1}$ we can rewrite (C.1) as

$$
s=\left(\begin{array}{cc}
1 & S_{1} \\
0 & 1
\end{array}\right)\left(\begin{array}{cc}
S_{2} & 0 \\
0 & S_{2}^{-1}
\end{array}\right)\left(\begin{array}{cc}
1 & 0 \\
S_{1} & 1
\end{array}\right) .
$$

This decomposition can also be written in terms of $S_{2}$ and $S=S_{1}+i S_{2}$ as

$$
s=i\left(\begin{array}{rr}
0 & 1 \\
-1 & 0
\end{array}\right)+\left(\begin{array}{cc}
0 & S^{\dagger} \\
0 & 1
\end{array}\right)\left(\begin{array}{cc}
S_{2}^{-1} & 0 \\
0 & S_{2}^{-1}
\end{array}\right)\left(\begin{array}{ll}
0 & 0 \\
S & 1
\end{array}\right) .
$$


Left multiplication on the group $G$ induces an action of the group $G$ on the coset space

$$
s^{\prime}=\left(\begin{array}{ll}
a & b \\
c & d
\end{array}\right) s\left(\begin{array}{ll}
a & b \\
c & d
\end{array}\right)^{\dagger} .
$$

Using the decomposition (C.2) one can easily show that the fractional transformation (6.19) of $S$ is equivalent to this action. The form (C.2) is very convenient since the first term is invariant under the action, while the second term only contains $S$ and $S_{2}$ and these have the simple transformation properties (6.19) and (6.21).

If we make all the matrices above real we obtain the parametrizations of $S p(2 n, \mathbb{R}) / U(n)$. If we change the basis with the unitary matrix $\mathcal{U}$ defined in (8.7) and then require all the matrices to be real we obtain the coset space $O(n, n) / O(n) \times O(n)$.

Since the map $\phi$ is injective we see that $S$, such that $S_{2}$ is positive definite, is a global coordinate on the coset space $U(n, n) / U(n) \times U(n)$. Thus this coset space is connected. The group $U(n, n)$ is a principal bundle over $U(n, n) / U(n) \times U(n)$ with a $U(n) \times U(n)$ fiber. The number of disconnected components of a principal bundle with a connected base is at most equal to the number of components of the fiber which in this case is one. Thus $U(n, n)$ is connected. Using the same argument one can show that $S p(2 n, \mathbb{R})$ is connected while $O(n, n)$ has at most four components. By an argument similar to the one used for the Lorentz group one can show that there are at least four components. Thus, as mentioned in Section 8.3, $O(n, n)$ has exactly four components. 


\section{Bibliography}

[1] E. Cremmer, B. Julia, The SO(8) Supergravity, Nucl. Phys. B159 (1979) 141

[2] S. Ferrara, J. Scherk, B. Zumino, Algebraic Properties of Extended Supergravity Theories, Nucl. Phys. B121 (1977) 393

[3] M. K. Gaillard, B. Zumino, Duality Rotations for Interacting Fields, Nucl. Phys. B193 (1981) 221

[4] B. Zumino, Quantum Structure of Space and Time, Eds. M. J. Duff and C. J. Isham (Cambridge University Press) (1982) 363

[5] M. Born, L. Infeld, Foundations of the New Field Theory, Proc. Roy. Soc. (London) A144 (1934) 425

[6] E. Schrödinger, Contributions to Born's New Theory of the Electromagnetic Field, Proc. Roy. Soc. (London) A150 (1935) 465

[7] G. W. Gibbons, D. A. Rasheed Electric-Magnetic Duality Rotations in NonLinear Electrodynamics, Nucl. Phys. B454 (1995) 185; hep-th/9506035

[8] G. W. Gibbons, D. A. Rasheed, $S L(2, R)$ Invariance of Nonlinear Electrodynamics Coupled to an Axion and Dilaton, Phys. Lett. B365 (1996) 46; hep-th/9509141 
[9] M. K. Gaillard, B. Zumino, Self-Duality in Nonlinear Electromagnetism, Supersymmetry and Quantum Field Theory, Kharkov 1997, J. Wess and V. P. Akulov, eds., Springer-Verlag 1998; hep-th/9705226

[10] M. K. Gaillard, B. Zumino, Nonlinear Electromagnetic Self-Duality and Legendre Transformations, Duality and Supersymmetric Theories, D. I. Olive and P. C. West, eds., Cambridge University Press 1999; hep-th/9712103

[11] J. Polchinski, Dirichlet-Branes and Ramond-Ramond Charges, Phys. Rev. Lett. 75 (1995) 4724-4727; hep-th/9510017

[12] P. K. Townsend, The eleven-dimensional supermembrane revisited, Phys. Lett. B350 (1995) 184-187

[13] E. Witten, String Theory Dynamics in Various Dimensions, Nucl. Phys. B443 (1995) 85-126; hep-th/9503124

[14] A. Strominger, Massless Black Holes and Conifolds in String Theory, Nucl. Phys. B451 (1995) 96-108; hep-th/9504090

[15] T. Banks, W. Fishler, S.H. Shenker, L Susskind, M Theory As A Matrix Model: A Conjecture, Phys. Rev. D55 (1997) 5112-5128; hep-th/9610043

[16] L. Susskind, Another Conjecture about M(atrix) Theory, hep-th/9704080

[17] A. Sen, Do Branes on $T^{n}$ and Matrix Theory, Adv. Theor. Math. Phys. 2 (1998) 51-59; hep-th/9709220 
[18] N. Seiberg, Why is the Matrix Model Correct?, Phys. Rev. Lett. 79 (1997) 3577-3580; hep-th/9710009

[19] A. Sen, An Introduction to Non-perturbative String Theory, hep-th/9802051

[20] E. Witten, Bound States of Strings and p-Branes, Nucl. Phys. B460 (1996) 335-350; hep-th/9510135

[21] M. R. Douglas, D. Kabat, P. Pouliot, S. H. Shenker, D-branes and Short Distances in String Theory, Nucl. Phys. B485 (1997) 85-127; hep-th/9608024

[22] M. Claudson, M. Halpern, Supersymmetric Ground State Wave Functions, Nucl. Phys. B250 (1985) 689

[23] R. Flume, On Quantum Mechanics with Extended Supersymmetry and Nonabelian Gauge Constraints, Ann. Phys. 164 (1985) 189

[24] M. Baake, P. Reinicke, V. Rittenberg, Fierz Identities for Real Clifford Algebras and the Number of Supercharges, J. Math. Phys. 26 (1985) 1070

[25] W. Taylor IV, D-brane Field Theory on Compact Spaces, Phys. Lett. B394 (1997) 283; hep-th/9611042

[26] O. J. Ganor, S. Ramgoolam, W. Taylor IV, Branes, Fluxes and Duality in M(atrix)-Theory, Nucl. Phys. 492, (1997) 191-204

[27] A. Connes, M. R. Douglas, A. Schwarz, Noncommutative Geometry and Matrix Theory: Compactification on Tori, JHEP02 (1998) 003; hep-th/9711162 
[28] M. R. Douglas, C. Hull, D-branes and the Noncommutative Torus, JHEP02 (1998) 008; hep-th/9711165

[29] P.-M. Ho, Y.-Y. Wu, Y.-S. Wu, Towards a Noncommutative Geometric Approach to Matrix Compactification, hep-th/9712201

[30] P.-M. Ho, Y.-S. Wu, Noncommutative Gauge Theories in Matrix Theory, hep-th/9801147

[31] R. Casalbuoni, Algebraic Treatment of Compactification on Noncommutative Tori, hep-th/9801170

[32] Y.-K. E. Cheung, M. Krogh, Noncommutative Geometry from 0-branes in a Background B-field, hep-th/9803031

[33] T. Kawano, K. Okuyama, Matrix Theory on Noncommutative Torus, hepth/9803044

[34] M. Rieffel, A. Schwarz, Morita equivalence of multidimensional noncommutative tori, math.QA/98033057

[35] F. Ardalan, H. Arfaei, M. M. Sheikh-Jabbari, Mixed Branes and M(atrix) Theory on Noncommutative Torus, hep-th/9803067

[36] P.-M. Ho, Twisted Bundle on Quantum Torus and BPS States in Matrix Theory, hep-th/9803166 
[37] D. Bigatti, Non commutative geometry and super Yang-Mills theory, hepth/9804120

[38] A. Schwarz, Morita equivalence and duality, hep-th/9805034

[39] G. Landi, F. Lizzi, R. J. Szabo, String Geometry and Noncommutative Torus, hep-th/9806099

[40] B. Morariu, B. Zumino, Super Yang-Mills on the Noncommutative Torus, hep-th/9807198

[41] F. Ardalan, H. Arfaei, M. M. Sheikh-Jabbari, Noncommutative Geometry from Strings and Branes, hep-th/9810072

[42] M. A. Rieffel, Induced Representations of $C^{*}$-algebras, Advances Math. 13 (1974) 176-257

[43] M. A. Rieffel, Morita Equivalence for Operator Algebras, Operator Algebras and Applications, (R. V. Kadison, ed.) Proc. Symp. Pure Math. 38, Amer. Math. Soc., Providence, 1982

[44] M. A. Rieffel, Projective Modules over Higher-Dimensional Noncommutative Tori, Can. J. Math., XL No. 2 (1988) 257-338

[45] M. A. Rieffel, The Cancellation Theorem for Protective Modules over Irrational Rotations C $^{*}$-Algebras, Proc. London Math. Soc. (3), 47 (1983) $285-302$ 
[46] M. A. Rieffel, Projective Modules over Higher-Dimensional Noncommutative Tori, Can. J. Math., XL No. 2 (1988) 257-338

[47] D. Brace, B. Morariu, B. Zumino, Dualities of the Matrix Model from T-Duality of the Type II String, hep-th/9810099

[48] D. Brace, B. Morariu, B. Zumino, T-Duality and Ramond-Ramond Backgrounds in the Matrix Model, hep-th/9811213

[49] A. Connes, M. Rieffel, Yang-Mills for noncommutative two-Tori, Operator Algebras and Mathematical Physics (Iowa City, Iowa, 1985) 237-266, Contemp. Math. Oper. Algebra. Math. Phys. 62, AMS 1987

[50] C. Hofman, E. Verlinde, U-Duality of Born-Infeld on the Noncommutative Two-Torus, hep-th/9810116

[51] C. Hofman, E. Verline, Gauge Bundles and Born-Infeld on the Noncommutative Torus, hep-th/9810219

[52] D. Brace, B. Morariu, A note on the BPS Spectrum of the Matrix Model, hep-th/9810185

[53] E. Witten, D. Olive, Supersymmetry Algebras that include Topological Charges Phys. Lett. 78B (1978) 97

[54] D. Brace, B. Morariu, B. Zumino, Duality Invariant Born-Infeld Theory, Yuri Golfand memorial volume; hep-th/9905218 
[55] I. Antoniadis, H. Partouche, T. R. Taylor, Spontaneous Breaking of N=2 Global Supersymmetry, Phys. Lett. B372 (1996); 83 hep-th/9512006

[56] M. Rocek, A. A. Tseytlin, Partial breaking of global D=4 supersymmetry, constrained superfields, and 3-brane actions, Phys. Rev. D59 (1999) 106001; hep-th/9811232

[57] S. Deser, R. Puzalowski, Supersymmetric Nonpolynomial Vector Multiplets and Causal Propagation, J. Phys. A13 (1980) 2501

[58] S. Cecotti, S. Ferrara, Supersymmetric Born-Infeld Lagrangians, Phys. Lett. 187B (1987) 335

[59] J, Bagger, A, Galperin, A New Goldstone Multiplet for a Partially Broken Supersymmetry, Phys. Rev. D55 (1997) 1091; hep-th/9608177

[60] Y. Tanii, N=8 Supergravity in Six-Dimensions, Phys. Lett. 145B (1984) 197

[61] S. Cecotti, S. Ferrara, L. Girardello, Hidden Noncompact Symmetries in String Theory, Nucl. Phys. B308 (1988) 436

[62] E. Cremmer, B. L. Julia, H. Lü, C. N. Pope, Dualisation of Dualities, I E II, Nucl.Phys. B523 (1998) 73, B535 (1998) 242; hep-th/9710119, hepth/9806106

[63] B. L. Julia, Dualities in the classical supergravity limits, in Strings Branes and Dualities, Cargese (1997), 121 hep-th/9805083 
[64] M. Araki, Y. Tanii, Duality Symmetries in Nonlinear Gauge Theories, Int. J. Mod. Phys. A14 (1999) 1139; hep-th/9808029

[65] N. Ishibashi, H. Kawai, Y. Kitazawa, A. Tsuchiya, A Large N Reduced Model as Superstring, Nucl. Phys. B498 (1997) 467-491

[66] A. Connes, Noncommutative Geometry, Academic Press, 1994

[67] D. Bigatti, Noncommutative Geometry for Outsiders, hep-th/9802129

[68] G. 't Hooft, A Property of Electric and Magnetic Flux in Nonabelian Gauge Theories, Nucl. Phys. B153 (1979) 141

[69] G. 't Hooft, Some Twisted Self-Dual Solutions for the Yang-Mills Equations on a Hypertorus, Commun. Math. Phys. 81 (

[70] W. Taylor IV, Lectures on D-branes, Gauge Theory and M(atrices), hepth/9801182

[71] A. Giveon, M. Porrati and E. Rabinovici, Target Space Duality in String Theory, Phys. Rept. 244 (1994) 77-202; hep-th/9401139

[72] E. Bergshoeff, C. M. Hull, T. Ortin, Duality in the Type-II Superstring Effective Action, Nucl. Phys. B451 (1995) 547-578; hep-th/9504081

[73] M. B. Green, C. M. Hull, P. K. Townsend, D-brane Wess-Zumino actions, T-duality and the cosmological constant Phys. Lett. B382 (1996) 65-72; hepth/9604119 
[74] E. Eyras, B. Janssen, Y. Lozano, 5-branes, KK-monopoles and T-duality, Nucl. Phys. B531 (1998) 275-301; hep-th/9806169

[75] A. Sen, Strong-Weak Coupling Duality in Four Dimensional String Theory, Int. J. Mod. Phys. A9 (1994) 3707-3750; hep-th/9402002

[76] M. Li, Boundary States of D-Branes and D-Strings Nucl. Phys. B460 (1996) 351; hep-th/9510161

[77] D. Berenstein, R. Corrado, J. Distler, On the Moduli Spaces of M(atrix)Theory Compactifications, Nucl. Phys. B503 (1997) 239-255; hep-th/9704087

[78] C. M. Hull, Matrix Theory, U-Duality and Toroidal Compactifications of M-Theory, hep-th/9711179

[79] C. Hofman, E. Verlinde, G. Zwart, U-Duality Invariance of the Fourdimensional Born-Infeld Theory, hep-th/9808128

[80] N. A. Obers, B. Pioline, E. Rabinovici M-theory and U-duality on $T^{d}$ with gauge backgrounds, Nucl. Phys. B525 (1998) 163; hep-th/9712084

[81] N. A. Obers, B. Pioline, U-duality and M-theory, hep-th/9809039

[82] B. Pioline, E. Kiritsis, U-duality and D-brane Combinatorics, Phys. Lett. B418 (1998) 61-69; hep-th/9710078

[83] A. Konechny, A. Schwarz BPS states on noncommutative tori and duality, hep-th/9811159 
[84] E. Witten, Constraints on Supersymmetry Breaking, Nucl. Phys. B202 (1982) 253

[85] P. Aschieri, D. Brace, B. Morariu, B. Zumino, Nonlinear Self-Duality in Even Dimensions, hep-th/9909021, to appear in Nuc. Phys. B

[86] B. Zumino, Supersymmetry and Kähler Manifolds, Phys. Lett. 87B (1979) 203

[87] P. Binetruy and M.K. Gaillard, S-Duality Constraints on Effective Potentials for Gaugino Condensation, Phys. Lett. B365 (1996) 87; hep-th/9506207 